\title{
EFFECTS OF THE USE OF ASSISTED REPRODUCTION AND HIGH CALORIC DIET CONSUMPTION ON BODY WEIGHT AND CARDIOVASCULAR HEALTH OF JUVENILE MOUSE OFFSPRING
}

\author{
A Thesis \\ Presented to the Faculty of the Graduate School \\ At the University of Missouri
}

In Partial Fulfillment

Of the Requirement for the Degree

Master of Science

By

ANGELA L. SCHENEWERK

Dr. Rocío Melissa Rivera and Dr. Luis A. Martínez-Lemus, Thesis Supervisors DECEMBER 2013 
The undersigned, appointed by the dean of the Graduate School, have examined the thesis entitled

\section{EFFECTS OF THE USE OF ASSISTED REPRODUCTION AND HIGH CALORIC DIET CONSUMPTION ON BODY WEIGHT AND CARDIOVASCULAR HEALTH OF JUVENILE MOUSE OFFSPRING}

Presented by Angela Louise Schenewerk

A candidate for the degree of Master of Science

And hereby certify that, in their opinion, it is worthy of acceptance.

Dr. Rocío Melissa Rivera

Dr. Luis A. Martínez-Lemus

Dr. Laura Schulz

Dr. Duane Keisler 


\section{ACKNOWLEDGEMENTS}

There are many people I would like to thank for helping me out in one way or another during my past two years here. First and foremost, I would like to thank my two major professors Dr. Rocío Rivera and Dr. Luis Martínez-Lemus for their patience with me, as I came here with very little laboratory experience. I could not have made it through this thesis work without their constant support and belief in me. I would also like to thank to thank the other two memebers of my committee, Dr. Laura Schulz and Dr. Duane Keisler for their valuable input for this new project. I would further like to thank the Miller Fellowhsip Committee for giving me the support to learn new, valuable research skills. Also, thank you to my undergraduate professors for helping me have the courage to try the new experience of a research lab.

Next, I would like to thank the current and previous members of the lab who added so much positivity to my experience here. I would like to thank Chen for always being there, whether it was for homework help or simply for telling jokes; Matt for his patience with me when initially trying to train me with the pipette; and Verónica for offering her support to me at any and ALL times. Further, I need to thank our undergraduates Laura Moone and Jasmine Randle. While I did not get to know them for long, they added a fresh, fun feeling in the lab. Thank you to Francisco and Christopher in the Martínez-Lemus lab for always lending me a helping hand when I needed one. I would also like to thank the other graduate students, especially Holly, Dane, Liz and Katy for always making me feel at home here.

I would also like to thank my family, and my soon-to-be family for being there for me in any way possible during my studies. Thank you to my fiancé Matt for always 
sending me his love and support, even from two hours away. A big thank you to my brother for giving me somewhere to go when I needed to be out of my apartment, and for allowing me to play with his kitties when I was missing mine from home. Last, but certainly not least, I want to thank my parents for their constant love, encouragement, and support no matter what the situation. I would truly not be where I am today without the help of all of the aforementioned people. 


\section{PREFACE}

Obesity and Cardiovascular Disease (CVD) are two current epidemics faced globally. While the onset of these diseases can be contributed partly to a person's lifestyle, the mechanism behind them is still largely unknown. The Developmental Origins of Health and Disease Hypothesis (DOHaD) asserts that the environment in which a fetus develops can affect the subsequent adult disease state. This was discovered from the initial association between maternal undernutrition and the increased risk for CVD in the offspring.

Two suboptimal maternal environments, maternal obesity and the environment formed with the use of assisted reproductive technologies (ART), have also been associated with increased risk of CVD in children. In this thesis, I will mainly describe how the $\mathrm{DOHaD}$ hypothesis has emerged to include maternal obesity and ART due to their increasing prevalence. Obesity in women is a risk factor for infertility; therefore, many obese women are now using ART to conceive. Both of these suboptimal environments for fetal development have been associated with adverse metabolic and cardiovascular outcomes in the offspring, including high blood pressure, also known as hypertension.

One mechanism for the development of hypertension associated with CVD is inward remodeling of the vasculature. This is where the smooth muscle cells reorganize, forming a smaller lumen and creating more resistance to blood flow. Based on previous research in our lab, Matrix metalloproteinases (MMPs) involved in the degradation of the extracellular matrix (ECM) are suggested to be involved in this inward remodeling. The 
activity of the MMPs can be increased by reactive oxygen species (ROS) derived from enzymes such as NADPH oxidase (NOX), and decreased by the endogenous tissue inhibitors of matrix metalloproteinases (TIMPs).

Studies have examined the effects of maternal obesity and ART separately on the subsequent disease state of the offspring, but data are lacking for the effect that the two environments have when they occur simultaneously. We hypothesize that obesity and ART independently and synergistically adversely affect the cardiovascular health and body weight of the offspring. We further hypothesize that these two suboptimal maternal environments will be associated with higher blood pressure in the offspring, and that this is mediated by the MMPs, TIMPs and NOXs. 


\section{TABLE OF CONTENTS}

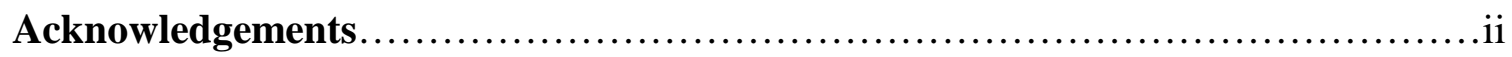

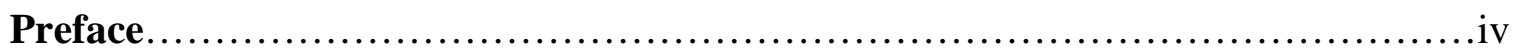

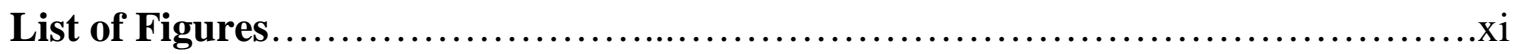

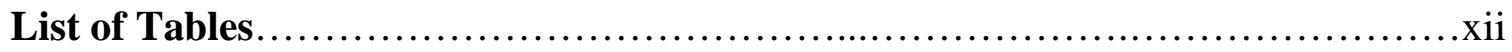

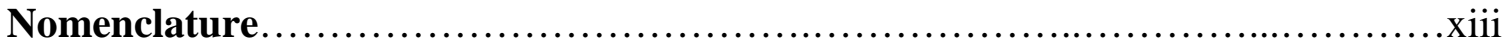

Chapter 1. Introduction of cardiovascular, metabolic and reproduction terms..........1

Cardiovascular terms.......................................................

Metabolic terms..........................................................

Reproduction terms.........................................................

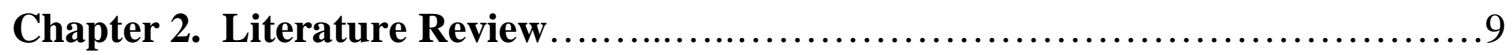

General introduction.......................................................

Cardiovascular disease..................................................11

Introduction to cardiovascular disease............................11

Introduction to the cardiovascular system.........................12

Hypertension and cardiovascular disease...........................14

Vascular development............................................17

Possible genes involved in poor cardiovascular health and hypertension development.................................................. 18

Introduction to the proposed mechanism of hypertension...............18

Matrix metalloproteinases.......................................18

Matrix metalloproteinase $2 \ldots \ldots \ldots \ldots \ldots \ldots \ldots \ldots \ldots \ldots . . \ldots \ldots$

Matrix metalloproteinase $9 \ldots \ldots \ldots \ldots \ldots \ldots \ldots \ldots \ldots \ldots .22$

Matrix metalloproteinase $7 \ldots \ldots \ldots \ldots \ldots \ldots \ldots \ldots \ldots \ldots . .23$ 
Tissue inhibitors of matrix metalloproteinases...................24

Tissue inhibitor of matrix metalloproteinase $1 \ldots \ldots \ldots . .24$

Reactive oxygen species and NADPH oxidase...................25

Reactive oxygen species..........................25

NADPH oxidase................................26

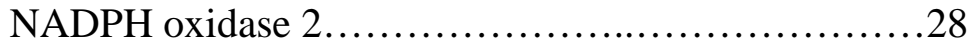

Relationship between ROS and MMPs.............................29

Conclusion..................................................33

Developmental origins of health and disease hypothesis....................33

Introduction to the $\mathrm{DOHaD}$ hypothesis.........................33

Barker's cardiovascular studies..................................34

Hertfordshire cohort study.............................35

Helsinki cohort studies.............................35

Dutch famine......................................36

Low birth weight, developmental plasticity and the

"thrifty phenotype"............................................38

Animal models of undernutrition.................................39

Obesity..........................................................42

Prevalence......................................................42

Effect of obesity and overweight on oocyte competence............42

Effect of obesity and overweight on preimplantation embryo development.................................................43

Adverse effects of obesity on post-implantation development.......44 
Maternal obesity/overweight and cardiovascular abnormalities in the offspring.... 46

Maternal consumption of a high fat diet..........................46

Effect of high fructose consumption...........................48

Assisted reproductive technologies......................................49

Introduction................................................49

ART procedures................................................49

Ovarian hyperstimulation.............................49

IVF and ICSI.....................................51

Embryo culture...................................51

Embryo transfer.................................53

Adverse consequences associated with ART.....................53

ART and cardiovascular abnormalities in offspring.........................................56

Maternal obesity and infertility and ART................................58

Maternal obesity and infertility..............................58

Reduced ART success with maternal obesity.....................59

Adverse pregnancy outcomes with maternal obesity and ART.......61

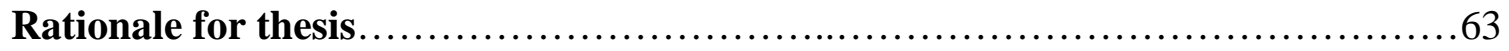

Chapter 3. Effects of the use of assisted reproduction and high caloric diet consumption on body weight and cardiovascular health of juvenile mouse

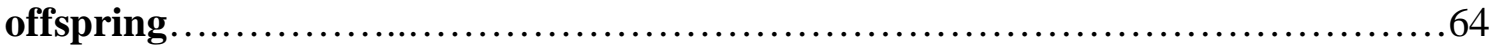

Abstract...............................................................64

Introduction.....................................................65

Materials and methods.................................................68 
Animals.

Diet.

Control (no ART) groups..................................69

Assisted reproduction groups.................................70

Superovulation....................................70

Embryo collection and culture......................70

Embryo transfers..................................71

Pregnancy, lactation and weaning.............................. 72

Measurement of offspring body weights.......................72

Mean arterial pressure measurement............................72

Mesenteric resistance artery and thoracic aorta collection............73

Dihydroethidium incubation of single mesenteric resistance arteries for detection of ROS.................................74

Fluorescence confocal microscopy for detection of intracellular ROS......................................................... 75

High Performance Liquid Chromatography (HPLC)...............76

Homogenizing vessels for protein measurement...................77

Protein assays using MicroBCA kit................................77

RNA extraction from thoracic aorta and RT-PCR.................78

Quantitative real-time PCR for gene expression in thoracic aorta.....79

Statistical analysis..........................................80

Results......................................................... 82

Effects of ART and diet on body weight from weaning to sacrifice...82

Effects of sex and diet on mean arterial pressure..................84 
Discussion........................................................90

Declaration of interest..................................................98

Funding.......................................................98

Acknowledgements..................................................98

Chapter 4. General discussion.................................................. 102

Bibliography......................................................... 111

Appendix 1. Design of bisulfite assays for section of promoter region in $\mathbf{M m p 2}$,

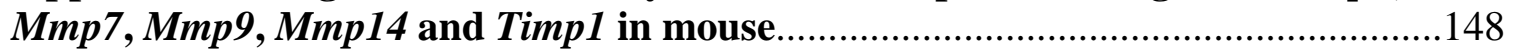

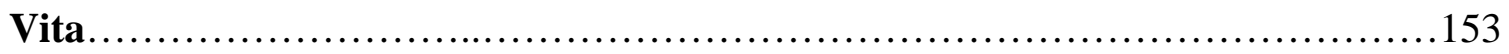




\section{LIST OF FIGURES}

Chapter 1. Introduction of cardiovascular, metabolic and reproduction terms

Figure 1.1. Diagram of vasculature commonly mentioned in the thesis............3

\section{Chapter 2. Literature Review}

Figure 2.1. Production of superoxide and hydrogen peroxide from oxygen........27

Figure 2.2. Involvement of MMPs, TIMPs, NOX and ROS in vascular remodeling.

Chapter 3. Effects of the use of assisted reproduction and high caloric diet consumption on body weight and cardiovascular health of juvenile mouse offspring

Figure 3.1. Effects of ART, diet and sex on mouse offspring body weight from weaning until seven weeks of age.

Figure 3.2. Mean arterial pressure (MAP) in offspring at sacrifice

Figure 3.3. Quantitative RT-PCR assessment of the expression of genes potentially involved in inward remodeling in offspring thoracic aortas.

Figure 3.4. Detection of extracellular and intracellular ROS in mesenteric resistance arteries of offspring

Supplementary Figure 3.1. Mean arterial pressure (MAP) in ART offspring and no ART controls at sacrifice.

Supplementary Figure 3.2. Quantitative RT-PCR measurement of the expression of genes potentially involved in inward remodeling in thoracic aorta of ART offspring and no ART controls

\section{Chapter 4. General Discussion}

Figure 4.1. Summary of significant effects on offspring cardiovascular health..105

\section{Appendix 1. Design of bisulfite assays for section of promoter region in Mmp2, Mmp7, Mmp9, Mmp14 and Timp1 in mouse}

Figure A1.1. Amplification of mouse bisulfite converted DNA.... 


\section{LIST OF TABLES}

Chapter 3. Effects of the use of assisted reproduction and high caloric diet consumption on body weight and cardiovascular health of juvenile mouse offspring

Table 3.1. TaqMan probes used for quantitative RT-PCR analyses in mouse thoracic aorta....................................................81

Table 3.2. Table of significant correlations between measurements in mouse offspring.

Supplementary Table 3.1. Births and number of offspring resulting from embryo

transfers

Appendix 1. Design of bisulfite assays for section of promoter region in $\mathbf{M m p 2}$, Mmp7, Mmp9, Mmp14 and Timp1 in Mouse

Table A1.1. PCR conditions for amplification of promoter regions in mouse bisulfite-converted DNA.

Table A1.2. Primer pair information for promoter regions in bisulfite-converted mouse DNA..............................................150 


\section{NOMENCLATURE}

AA

AAA

ACE

ADA

ANGII

ART

BMI

BSA

CDC

CHR

CVD

DOHaD

eCG

ECM

FOAD

FSH aortic aneurysm

abdominal aortic aneurysm

angiotensin converting enzyme

American Diabetes Association

angiotensin II

Assisted Reproductive Technologies

body mass index

bovine serum albumin

Centers for Disease Control

Center for Human Reproduction

Cardiovascular disease

Developmental Origins of Health and Disease

equine chorionic gonadotropin

extracellular matrix

Fetal Origins of Adult Disease

follicle stimulating hormone 


$\begin{array}{ll}\text { GnRH } & \text { gonadotropin releasing hormone } \\ \mathrm{H}_{2} \mathrm{O}_{2} & \text { hydrogen peroxide } \\ \mathrm{HF} & \text { high fat (includes high fructose in Ch III) } \\ \text { hMG } & \text { human menopausal gonadotropin } \\ \text { ICSI } & \text { intracytoplasmic sperm injection } \\ \text { IDF } & \text { International Diabetes Foundation } \\ \text { IL-6 } & \text { interleukin-6 } \\ \text { IVF } & \text { in vitro fertilization } \\ \text { IU } & \text { international units } \\ \text { LGA } & \text { large for gestational age } \\ \text { LH } & \text { metaphase II } \\ \text { LOI } & \text { luteinizing hormone } \\ \text { MAP } & \text { mean arterial pressure } \\ \text { MII } & \text { mprinting } \\ & \end{array}$

MMP1/2/7/8/9 matrix metalloproteinase 1/2/7/8/9

MS Metabolic Syndrome

NCBI National Center for Biotechnology Information 
NCI

NE

NEFA

NFKB

NO

NOX

NOX2

$\mathrm{O}_{2}{ }^{-}$

PPAR $\alpha$

PUFA

RAS

ROS

SART

SBP

SO

SOD

T2DM

TIMPs 1-4
National Cancer Institute

norepinephrine

nonesterified fatty acid

nuclear factor kappa-light-chain-enhancer of activated B cells

nitric oxide

NADPH oxidase

NADPH oxidase 2

superoxide

peroxisome proliferator-responsive element $\alpha$

polyunsaturated fatty acid

renin-angiotensin system

reactive oxygen species

Society for Assisted Reproductive Technologies

systolic blood pressure

superovulation

superoxide dismutase

Type II Diabetes Mellitus

tissue inhibitor of matrix metalloproteinase 1-4 
WHF

WHO
World Heart Federation

World Health Organization 


\section{CHAPTER 1}

\section{Introduction of Cardiovascular, Metabolic and Reproduction Terms}

This section aims to orient the reader to some of the cardiovascular, metabolic and reproduction terms that are helpful in fully understanding the variety of topics presented in this thesis. Definitions are listed verbatim or nearly verbatim and the proper source is accredited.

\subsection{Cardiovascular Terms}

Cardiovascular Disease- Cardiovascular diseases (CVDs) are a group of disorders of the heart and blood vessels and they include:

- coronary heart disease - disease of the blood vessels supplying the heart muscle

- cerebrovascular disease - disease of the blood vessels supplying the brain

- $\quad$ peripheral arterial disease - disease of blood vessels supplying the arms and legs

- $\quad$ rheumatic heart disease - damage to the heart muscle and heart valves from rheumatic fever, caused by streptococcal bacteria

- congenital heart disease - malformations of heart structure existing at birth

- deep vein thrombosis and pulmonary embolism - blood clots in the leg veins, which can dislodge and move to the heart and lungs.

(WHO 2013b)

Atherosclerosis- the process in which deposits of fatty substances, cholesterol, cellular waste products, calcium and other substances build up in the inner lining of an artery (AHA 2013).

Aortic abdominal aneurysm- An aortic aneurysm (AA) is a ballooning or dilatation of the aorta, the large artery that carries blood from the heart through the chest and abdomen. AAs are classified according to their location; in the chest, it is called a thoracic AA, in the abdomen an abdominal AA (AAA), and across both areas a 
thoracoabdominal AA [ see Fig. 1.1 for thoracic aorta and abdominal aorta; (CDC 2011)].

Mean Arterial Pressure (MAP)- MAP, the product of cardiac output (CO) and total peripheral resistance (TPR), is the time-averaged blood pressure within the arterial circuit, as well as the average pressure in the arteries throughout the cardiac cycle (Henry et al. 2002).

Systolic Blood Pressure (SBP)- In a blood pressure measurement (i.e. 120/80), the upper number ("120”) is the systolic blood pressure, which is the highest pressure in blood vessels and happens when the heart contracts, or beats. Normal blood pressure is 120/80 (WHO 2013d).

Diastolic Blood Pressure (DBP)- The lower number (“80”) is the diastolic blood pressure, which is the lowest pressure in blood vessels in between heartbeats when the heart muscle relaxes (WHO 2013d).

Hypertension- a systolic blood pressure equal to or above $140 \mathrm{~mm} \mathrm{Hg}$ and/or diastolic blood pressure equal to or above $90 \mathrm{~mm} \mathrm{Hg}$ (WHO 2013d). 


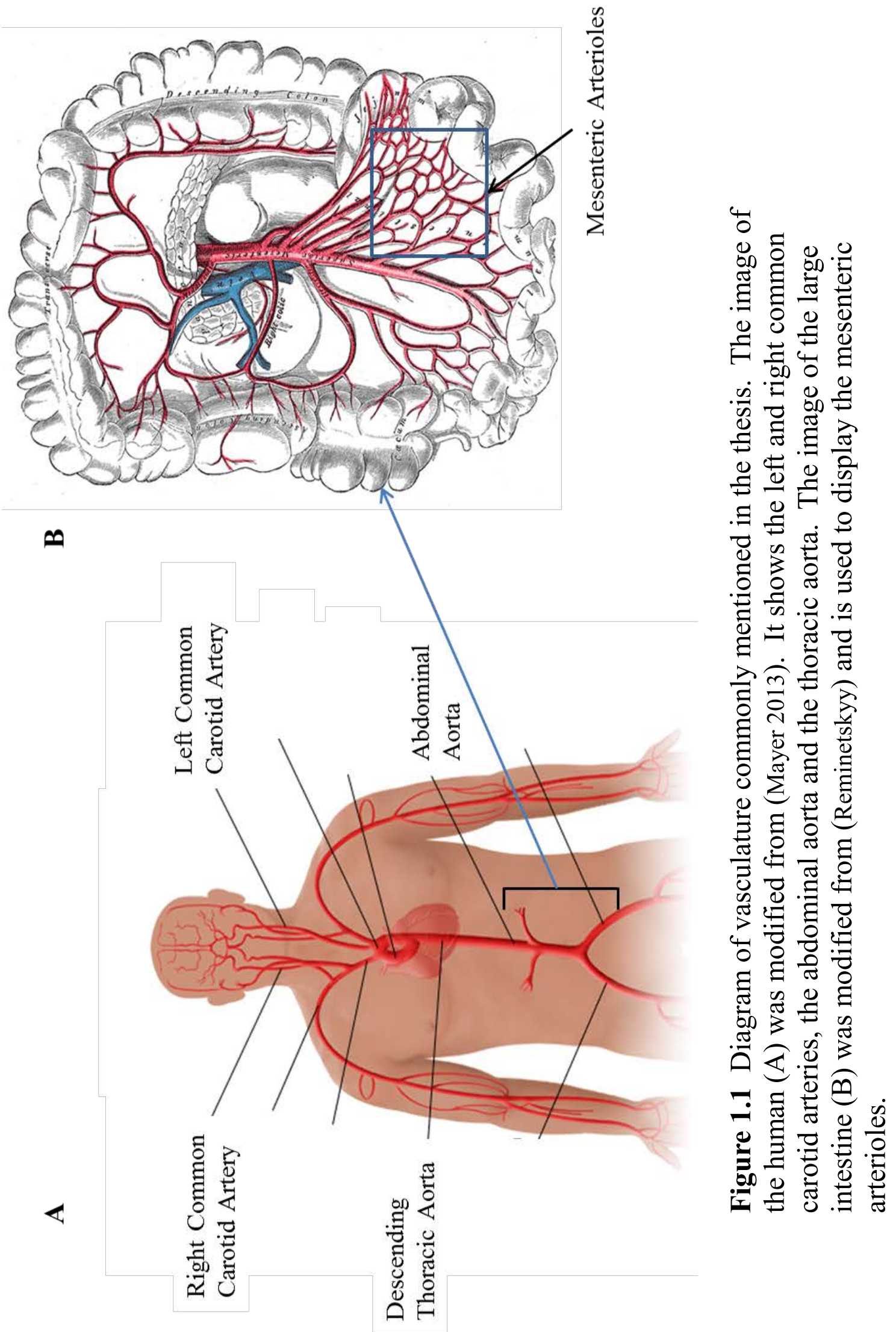


Renin-Angiotensin System (RAS)- a peptidergic system with endocrine characteristics. The substrate of the system, angiotensinogen, an $\alpha$-glycoprotein, is released from the liver and is cleaved in the circulation by the enzyme renin that is secreted from the juxtaglomerular apparatus of the kidney to form the decapeptide angiotensin (ANG) I. ANG I is then activated to the octapeptide ANG II by angiotensin converting enzyme (ACE), a membrane-bound metalloproteinase, which is predominantly expressed in high concentrations on the surface of endothelial cells in the pulmonary circulation. ANG II, considered the main effector peptide of the RAS, then acts on specific receptors (Paul et al. 2006).

Inward eutrophic remodeling- inward, eutrophic remodeling of arterioles does not involve cellular proliferation and thus it is due to rearrangement of the same vascular smooth muscle cells (VSMCs) around a smaller lumen (Prewitt et al. 2002)

Endothelial Dysfunction- The term endothelial dysfunction has been used to refer to several pathological conditions, including altered anticoagulant and anti-inflammatory properties of the endothelium, impaired modulation of vascular growth, and dysregulation of vascular remodeling. However, in much of the literature this term has been used to refer to an impairment of endothelium-dependent vasorelaxation caused by a loss of nitric oxide (NO) bioactivity in the vessel wall (Cai \& Harrison 2000).

Coronary Heart Disease/Coronary Artery Disease/Ischemic Heart Disease- Disease of the blood vessels supplying the heart muscle (WHO 2013a).

Extracellular Matrix- The extracellular matrix (ECM) is the noncellular component present within all tissues and organs, and provides not only essential physical scaffolding 
for the cellular constituents but also initiates crucial biochemical and biomechanical cues that are required for tissue morphogenesis, differentiation and homeostasis (Frantz et al. 2010).

\subsection{Metabolic Terms}

Macrosomia- Macrosomia is a term mostly used for newborns with a birthweight in weight units above a certain limit. However, there is no general agreement what this limit should be. Human birth weights above 4,000, 4,200 and 4,500 $\mathrm{g}$ are being used as definitions of newborn macrosomia (Henriksen 2008).

Body mass index (BMI)- Body Mass Index (BMI) is a simple index in humans of weight-for-height that is commonly used to classify underweight, overweight and obesity in adults. It is defined as the weight in kilograms divided by the square of the height in metres [kg/m²; (WHO 2006)].

Overweight and Obesity- Overweight and obesity are defined as abnormal or excessive fat accumulation that may impair health. The WHO definition is:

- a BMI greater than or equal to 25 is overweight

- a BMI greater than or equal to 30 is obesity.

(WHO 2013e)

Morbidly obese is a BMI greater than or equal to 40 (Dokras et al. 2006). Type II Diabetes Mellitus- This form of diabetes, which accounts for $\sim 90-95 \%$ of those with diabetes, previously referred to as non-insulin-dependent diabetes, type 2 diabetes, or adult-onset diabetes, encompasses individuals who have insulin resistance and usually have relative (rather than absolute) insulin deficiency (ADA 2013). 
Glucose Intolerance (impaired glucose tolerance)- Impaired glucose tolerance (IGT), along with impaired fasting glucose (IFG), is recognised as being a stage before diabetes when blood glucose levels are higher than normal (IDF 2013).

Hyperinsulinemia- Hyperinsulinemia is defined in the presence of fasting insulin levels greater than 30 micro units $(\mu \mathrm{U}) / \mathrm{ml}$ or when the sum of insulinemic values at the second and third hours of the glycemic curve is greater than $60 \mu \mathrm{U} / \mathrm{ml}$ (Lavinsky et al. 2004).

Hyperleptinemia- elevated plasma leptin levels (Ren 2004).

Metabolic Syndrome- There is no internationally agreed definition for the Metabolic Syndrome. The following, which does not imply causal relationships, is suggested as a working definition: glucose intolerance, or diabetes mellitus and/or insulin resistance together with two or more of the other components listed below.

1. Impaired glucose regulation or diabetes

2. Insulin resistance (under hyperinsulinaemic euglycaemic conditions, glucose uptake below lowest quartile for background population under investigation)

3. Raised arterial pressure $\geq 160 / 90 \mathrm{mmHg}$

4. Raised plasma triglycerides $\left(\geq 1.7 \mathrm{mmol}^{-1} ; 150 \mathrm{mg} \mathrm{dl}^{-1}\right)$ and/or low HDL-cholesterol ( $<0.9 \mathrm{mmol} \mathrm{l}^{-1}, 35 \mathrm{mg} \mathrm{dl}^{-1} \mathrm{men} ;<1.0 \mathrm{mmol} \mathrm{l}^{-1}, 39 \mathrm{mg} \mathrm{dl}^{-1}$ women$)$

5. Central obesity (males: waist to hip ratio $>0.90$; females: waist to hip ratio $>0.85$ ) and/or BMI $>30 \mathrm{~kg} \mathrm{~m}^{-2}$

6. Microalbuminuria (urinary albumin excretion rate $\geq 20 \mathrm{mg} \mathrm{min}^{-1}$ or albumin:creatinine ratio $\geq 20 \mathrm{mg} \mathrm{g}^{-1}$ )

(Alberti \& Zimmet 1998)

Gestational Diabetes Mellitus- any degree of glucose intolerance with onset or first recognition during pregnancy (ADA 2013). 


\subsection{Reproduction Terms}

Follicle Stimulating Hormone (FSH)- FSH (follicle stimulating hormone) is a hormone released by the pituitary gland which stimulates the growth of follicles and has a role in the maturation of oocytes (Gleicher 2013).

Luteinizing Hormone (LH)- LH is produced by gonadotropic cells in the anterior pituitary gland. In females, a sharp rise in LH triggers ovulation and development of the corpus luteum (Choi et al. 2013).

Anovulation- Disorders of anovulation account for about 30\% of infertility and often present with irregular periods (oligomenorrhea) or an absence of periods [amenorrhea; (Katsikis et al. 2006)].

Preeclampsia- Preeclampsia refers to a syndrome characterized by the new onset of hypertension and proteinuria after 20 weeks of gestation in a previously normotensive woman (Ghulmiyyah \& Sibai 2012).

Syncytiotrophoblast- The syncytiotrophoblasts are a continuous, specialized layer of uterine epithelial cells. They cover the entire surface of villous trees and are in direct contact with maternal blood (Wang 2010).

Assisted Reproductive Technologies (ART)- ART includes all fertility treatments in which both eggs and sperm are handled. In general, ART procedures involve surgically removing eggs from a woman's ovaries, combining them with sperm in the laboratory, and returning them to the woman's body or donating them to another woman (CDC 2012). 
Female Infertility- In general, infertility is defined as not being able to get pregnant (conceive) after one year of unprotected sex (CDC 2013).

In vitro fertilization (IVF)- IVF involves extracting a woman's eggs, fertilizing the eggs in the laboratory, and then transferring the resulting embryos into the woman's uterus through the cervix (CDC 2012).

Intracytoplasmic sperm injection (ICSI)- A procedure in which a single sperm is injected directly into an egg; this procedure is commonly used to overcome male infertility problems (CDC 2012).

Embryo transfer- Placement of embryos into a woman's uterus through the cervix after IVF (CDC 2012).

Ovarian Stimulation-The use of drugs (oral or injected) to stimulate the ovaries to develop follicles and eggs (CDC 2012). 


\section{CHAPTER 2}

\section{Literature Review}

\subsection{General Introduction}

CVD is the leading cause of death both in the United States and world-wide. According to World Health Organization (WHO) data, it is estimated that CVD will account for as many as 23.3 million deaths annually by 2030. Despite the increasing prevalence of the disease, the mechanism behind the development of CVD is largely unknown.

Low birth weight can be attributed to poor maternal nutrition and has been associated with an increased risk for CVD. Therefore, both human and animal models of maternal undernutrition have been used to examine the effect of maternal nutrition on offspring CVD. The hypothesis that the environment an individual is exposed to while in utero can affect the individual's subsequent disease state in adulthood became known as the Fetal Origins of Adult Disease (FOAD), and more recently renamed the Developmental Origins of Health and Disease (DOHaD) hypothesis.

While originally the DOHaD hypothesis was used to describe the effects of maternal undernutrition on offspring, it now encompasses other suboptimal maternal environments including maternal obesity and overweight. Obesity is an epidemic both in the United States and world-wide. Over 300 million women and 200 million men in 2008 were considered obese by the World Health Organization. Further, over 64\% of women of reproductive age in the United States are overweight. Assisted reproductive technologies (ART) also provide an altered environment for fetal development. ART is 
used to overcome infertility which affects approximately 10 percent of women ages 1544 in the United States. While most children conceived with the use of ART are healthy, both maternal obesity and the use of ART have been associated with adverse outcomes in the children such as increased peripheral body fat, lowered glucose tolerance and increased systolic blood pressure associated with hypertension.

A proposed mechanism of hypertension involves inward remodeling of the vasculature, resulting in increased resistance to blood flow. Previous work in our laboratory has suggested that this inward remodeling is a result of increased activity of matrix metalloproteinases (MMPs). Both obesity and the use of ART can result in increased oxidative stress. Oxidative stress has been associated with up regulated production of reactive oxygen species (ROS) by NADPH Oxidase (NOX) which can result in increased activity of the MMPs. MMP activity can be decreased by the tissue inhibitors of matrix metalloproteinases (TIMPs).

Women who are obese have an increased risk for infertility, leading many of these women to use ART in order to conceive. There are many studies that examine the effects of these two environments alone, but knowledge is lacking on what effect these two maternal environments have on the disease state of the offspring when used in combination. For my thesis research, I studied the effects of these two suboptimal maternal environments on body weight and blood pressure in mouse offspring and sought to determine whether a mechanism involving the MMPs, TIMPs and NOX-derived ROS is involved in increased offspring blood pressure. 
This literature review will provide background information that is helpful for fully understanding the information presented in the research presented in Chapter 3. The topics covered include CVD, the $\mathrm{DOHaD}$ hypothesis, and current knowledge about the effects of maternal overweight/obesity and ART on offspring development and subsequent disease state.

Note: The standards about the use of capital and lower case letters when writing about a gene, RNA or protein can vary between species and between journals (i.e. MMP2 vs. Mmp2 for RNA in humans and mice, respectively). The research conducted as part of this thesis was prepared for submission to the journal Reproduction. For simplicity, the gene and protein nomenclature will be presented in the format required by Reproduction throughout the entire thesis. Reproduction requires that all genes/DNA/RNA be italicized and in all capital letters for humans, non-human primates and domestic species (i.e. MMP2) and with only the first letter capitalized in mice and rodents (i.e. Mmp2). Protein nomenclature for all of the above-mentioned species is the same, with all letters capitalized and no italics (i.e. MMP2). If I am referring to the group as a whole (i.e. the matrix metalloproteinases), I will list it in all capital letters (i.e. the MMPs).

\subsection{Cardiovascular Disease}

\section{a) Introduction to Cardiovascular Disease}

The number one cause of death world-wide is Cardiovascular Disease (CVD), which accounted for nearly 30\% of deaths in 2008 (WHO 2013b). Most of the deaths from CVD occur in low- and middle- income countries. The incidences of CVD are 
becoming more common and it is estimated that CVD will globally account for 23.3 million deaths per year by 2030 (WHO 2013b). Obesity and high blood pressure are two common risk factors for CVDs. Some common forms of CVDs include coronary, peripheral, and cerebrovascular diseases of the vessels supplying the heart, arms and legs, and brain; respectively (WHO 2013b). Heart attack and stroke are two other acute forms of CVD which occur most commonly due to lipid buildup in the arteries (WHO 2013b). CVD in children also includes congenital heart disease, which is present from birth (WHF 2012). The global epidemic of obesity, which will be discussed later, has also led to an increase in the prevalence of cardiovascular risk factors in children with the potential to lead to premature CVD (WHF 2012).

\section{b) Introduction to the Cardiovascular System}

To understand CVD and its pathologies, one must first understand how the cardiovascular system functions physiologically. While the main focus of this section will be on the arteries, which carry blood away from the heart to supply nutrients to the tissues, I will first briefly mention the cardiovascular system as a whole. The cardiovascular system is also known as the circulatory system due to its role of circulating blood throughout the body. The system consists of the heart and the vasculature, including the arteries, veins, capillaries and lymphatics. Blood is transported by the arteries to the tissues of the body to supply oxygen and other nutrients, and the veins transport deoxygenated blood back to the heart and then the lungs to become reoxygenated (Barrett 2012). The capillaries are where the nutrient exchange occurs in the tissues and the lungs (NCI 2012). The amount of nutrients that reach the tissues depends both on the functionality of the heart and of the vasculature. Blood flow 
is directed with each heart beat by a pressure gradient from the large blood vessels near the heart to the smaller peripheral vessels (London and Pannier, 2010).

The arterial system consists of both macrovasculature including the aorta and the large arteries, and the microvasculature which includes the small arteries, arterioles and capillaries (Barrett 2012). The aorta and larger arteries near the heart contain large amounts of elastin and are distensible to accommodate large amounts of blood with each pump of the heart (Hendry et al. 2012). The macrovasculature can hypertrophy and become stiffer and more resistant to flow (Briet \& Schiffrin 2013). This has been associated with increased risk for death due to cardiovascular events (Vlachopoulos et al. 2010). However, the main focus of this review will be on the microvasculature which is the primary source of resistance to blood flow (Barrett 2012).

The small arteries and arterioles provide most of the resistance in the cardiovascular system, and they are where the main structural changes attributed to increased peripheral resistance occur (Mulvany \& Aalkjaer 1990). Microvasculature includes the blood vessels with diameters smaller than $150 \mu \mathrm{M}$, and its main function is to transport nutrients and oxygen in accordance to the demand of the tissues (Clough and Norman, 2011). From the outermost to the innermost layers, the arteries include the adventitia made of connective tissue, the media composed of smooth muscle and the intima which includes the endothelium and connective tissue (Ross \& Glomset 1976). The microvasculature has more smooth muscle in its walls, and is capable of a larger change in diameter than the macrovasculature (Hendry et al. 2012). 
One of the contributing factors to the amount of blood and nutrients that reach the tissues is total peripheral resistance. Peripheral resistance is determined largely by the diameter of the blood vessels (Hendry et al. 2012). The diameter of the vasculature can change through a narrowing (vasoconstriction) or an increase in the diameter of the blood vessel [vasodilation; (Barrett 2012)].

Vasoconstriction and vasodilation can occur due to various stimuli including mechanical stretch, sympathetic nerve activities, neurohormonal agents and endothelialderived products. Stretch due to increased blood pressure can be sensed by baroreceptors which respond by decreasing sympathetic nerve activity, resulting in vasodilation (Green \& Heffron 1968). Norepinephrine (NE) and Angiotensin II (ANGII) are two neurohormonal agents that are potent vasoconstrictors. An endothelial product that can increase the diameter of the blood vessels is nitric oxide (NO), which is a potent vasodilator (Barrett 2012). In physiological conditions, vasoconstriction and vasodilation create a blood supply to the tissues that is nearly equivalent to that tissue's oxygen demand (Saltin et al. 1998). However, vasoconstriction and vasodilation can also become pathophysiological as will be discussed later.

\section{c) Hypertension and Cardiovascular Disease}

Systolic blood pressure (SBP; the maximum pressure in the arteries during contraction of the heart) above $140 \mathrm{mmHg}$ and diastolic blood pressure (the maximum pressure in the arteries during relaxation of the heart) above $90 \mathrm{mmHg}$ is considered hypertension (WHO 1999). Over 16\% of deaths each year are attributed to hypertension (WHO 2013b). Cardiovascular complications due to the effects of hypertension are vast 
and include aneurysms, hypertrophy of the left ventricle, hemorrhage and renal failure (Hollander 1976). Lowering blood pressure in hypertensive patients has been associated with decreased morbid cardiovascular outcomes (Taguchi \& Freis 1975, Czernichow et al. 2011).

Hypertension has been shown to increase the rate of atherosclerosis (thickening of vessel walls due to fatty deposits) development, especially in the macrovasculature (Robertson \& Strong 1968). This has been demonstrated in a model of non-human primates where hypertension accelerated the development of atherosclerosis when compared to primates without hypertension (Hollander et al. 1993). Hypertension can also result in hypertensive vascular disease, which creates a narrowing of the lumen in the arteries (Hollander 1976). The lesions formed in hypertensive vascular disease are different than those found in atherosclerosis because these lesions are formed without the presence of excess lipids (Hollander et al. 1968). Instead, they are associated with increased mucopolysaccharides, electrolytes and water in the media of the vessels.

Increase of vascular resistance, or the resistance that the vasculature provides against the flow of blood, in all major organ systems is one of the defining characteristics in hypertension (Heagerty et al. 1993). Many mechanisms have been proposed to be involved in the development of hypertension. One such mechanism involves the reninangiotensin system (RAS) where a study revealed that blocking the conversion of Angiotensin I to the potent vasoconstrictor ANGII by Angiotensin Converting Enzyme (ACE) lowered blood pressure in spontaneously hypertensive rats (Antonaccio et al. 1979). Blood pressure reduction by inhibition of the RAS has also been observed in humans (Herrera-Acosta et al. 1985, Ajayi et al. 1986). 
Mean arterial pressure (MAP) is determined by cardiac output and total peripheral resistance, so increasing total peripheral resistance can cause an increase in MAP (London \& Pannier 2010). As mentioned above, the arterioles are the main resistance vessels, so they are a main contributor to the total peripheral resistance. Increased peripheral resistance and a diminished vasodilation response to flow in the microcirculation has been associated with early hypertension (Schwartzkopff et al. 1993). Too much impedance to blood flow due to vascular resistance can result in pressure build-up towards the heart, forcing the heart to work harder with each pump (London and Pannier, 2010). Hypertension has been associated with a decreased vasodilatory response to acetylcholine, showing a decrease in endothelial function (Rizzoni et al. 1996). The impairment of vasodilation in resistance vessels can also result in inward eutrophic remodeling (Pistea et al. 2005).

Inward eutrophic remodeling occurs when the already existing smooth muscle cells rearrange creating a smaller lumen (Heagerty et al. 1993, Rizzoni et al. 2003a). Models of hypertension have shown that the inward remodeling that occurs in the arterioles is eutrophic (Korsgaard et al. 1993, Rizzoni et al. 1996). That is, there is a reduction in the passive luminal diameter of the vessels without significant changes in the cross-sectional area, represented by the amount of material in the vascular wall [reviewed in (Mulvany 1999)]. The inward eutrophic remodeling of the arterioles has been associated with increased risk of life-threatening cardiovascular events such as stroke or myocardial infarction (Rizzoni et al. 2003b, Mathiassen et al. 2007). This inward remodeling could also be a result of increased RAS activity and sympathetic nerve 
activation, as prolonged exposure to NE+ANGII has been shown to result in inward remodeling in arterioles (Martinez-Lemus et al. 2011).

\section{d) Vascular Development}

The cardiovascular system is the first physiological system to develop in the fetus, so it is prone to damage from early insults in the maternal environment (Clough and Norman, 2011). The placenta is responsible for delivering nutrients to and removing wastes from the fetus [reviewed in (Maltepe et al. 2010)]. Angiogenesis, or the formation of new blood vessels, is an important process during placentation allowing for rapid growth of the developing fetus (Maltepe et al. 2010). Once implantation occurs, blood flow to the placenta is established by remodeling of the maternal spiral arteries [reviewed in (Red-Horse et al. 2004)]. The spiral arteries provide blood to the placental intervillous space starting at about 12 weeks of human pregnancy (Burton et al. 1999). Due to the delayed maternal blood flow, the fetus and placenta develop in a low oxygen environment during the first trimester of pregnancy (Watson et al. 1998).

The placental vasculature is not innervated, therefore its tone depends on the release of vasodilators and vasoconstrictors (Fox \& Khong 1990). The tone of the vasculature in the placenta can be controlled by stimuli such as the RAS and the potent vasodilator NO (Wang 2010). The fetal vascular bed in the placenta has low vascular resistance, allowing for high blood flow to the developing fetus (Trudinger et al. 1985). After birth the infant's vascular resistance rises to regulate the blood flow as in adults (Barrett 2012). In the placenta, the arteries stem from the fetus carrying deoxygenated blood to a mass of capillary loops associated with the syncytiotrophoblast which connects 
the fetus to the mother. This is where the blood becomes oxygenated before returning to the fetus through a vein within the umbilicus (Wang 2010). The blood flow provides the source of nutrients for the developing fetus, so an impairment in the blood flow in the placenta (i.e. decreased vasodilation due to decreased NO availability) can adversely affect fetal development (Wang 2010). The RAS is also present in the placenta, and higher placental ANGII receptor AT1R levels have been found in pregnancies complicated by preeclampsia (Mistry et al. 2013).

\subsection{Possible Genes Involved in Poor Cardiovascular Health and Hypertension Development}

\section{a) Introduction to the Proposed Mechanism of Hypertension}

It has previously been shown that inward eutrophic remodeling occurs in hypertension (Korsgaard et al. 1993, Rizzoni et al. 2003b). Also, it has recently been proposed that the development of hypertension can be mediated by vascular remodeling induced by oxidative stress and the increased activity of matrix metalloproteinases [MMPs; (Martinez-Lemus et al. 2011)]. This section will summarize how reactive oxygen species (ROS) and MMPs have been associated with poor cardiovascular health and how they might be involved in the vascular remodeling associated with hypertension.

\section{b) Matrix Metalloproteinases}

The MMPs are part of a family of zinc-dependent endopeptidases, and one source of their production is cells in the walls of blood vessels (Raffetto \& Khalil 2008). MMPs 
were first discovered by Gross and Lapiere in 1962 for their involvement in the dissolution of structures such as the tail and gills in tadpole morphogenesis (Gross \& Lapiere 1962). The 23 MMPs found in humans are subdivided into five groups, namely the collagenases, gelatinases, matrilysins, stromelysins, and membrane-type MMPs. These groups are classified by their substrates and sequence homologies [reviewed in (Lindsey \& Zamilpa 2012)]. MMP expression is usually low in most tissues, but expression can be upregulated in several ways, such as by inflammatory cytokines and growth factors (Nagase \& Woessner 1999).

The MMPs are secreted as inactive pro-MMPs, and the inactive state is maintained by a cysteine residue found in the propeptide domain that ligates the catalytic zinc [i.e. the "cysteine switch"; (Van Wart \& Birkedal-Hansen 1990)]. The cysteine switch refers to the ability of the MMP to be activated when the propeptide domain is removed and the cysteine residue no longer blocks the activity of the catalytic domain (Van Wart \& Birkedal-Hansen 1990). The catalytic domain has a zinc binding motif and the structure is maintained by a structural zinc ion and calcium ions (Bode et al. 1993). Most MMPs also contain a $C$-terminal hemopexin-like domain which is required for the cleavage of triple helical collagen (Bode 1995). However, this hemopexin-like domain is absent in the matrilysin MMP7 (Gaire et al. 1994). Activation of MMPs occurs when the cysteine switch is disrupted and the pro-domain is removed [reviewed in (Nagase 1997)]. MMPs can be activated by proteinases (including other MMPs) as well as by sulfhydryl reactive agents like oxidized glutathione and by denaturants such as urea (Nagase 1997).

MMPs are best known for their role in the degradation of the extracellular matrix (ECM), but they also participate in the cleavage of extracellular receptors (e.g. the $\beta 2$ 
adrenergic receptor) resulting in reduced vasodilation (Rodrigues et al. 2010), disruption of cellular tight junctions (Yang et al. 2007), and shedding of vasoactive factors associated with the ECM (e.g. heparin-binding epidermal growth factor) which is then able to interact with the epidermal growth factor receptor to promote vasoconstriction (Hao et al. 2004). They have physiological roles such as involvement in placentation (Onogi et al. 2011) and wound healing (Salo et al. 1994), but they have also been implicated in pathological conditions like cancer (Friedberg et al. 1998) and stroke (Cojocarui et al. 2012).

In the vasculature, MMPs are involved in vasoconstriction as well as reduction in vasodilation. MMPs can degrade the ECM allowing for movement of the smooth muscle cells during vascular remodeling (Martinez-Lemus \& Galinanes 2011). They can also cleave $\beta 2$ adrenergic receptors, resulting in decreased vasodilation (Rodrigues et al. 2010). This can induce hypertension which is associated with inward remodeling, so it has been proposed that the MMPs might be involved in inward eutrophic remodeling through their vasomotor activities (Martinez-Lemus \& Galinanes 2011).

In support of this idea, it has been shown that the activity of MMPs in the plasma is elevated in hypertension (Friese et al. 2009). It has also been shown that inward remodeling induced by NE and ANGII (potent vasoconstrictors) was prevented when MMPs were inhibited (Martinez-Lemus et al. 2011). The MMPs that are of particular interest in the studies of hypertension are MMP2, MMP9, and MMP7, which will be discussed below. 


\section{Matrix Metalloproteinase 2}

MMP2 is a type IV collagenase/gelatinase A that was first described in the 1970's (Sellers et al. 1978). The gene encoding MMP2 is found on chromosome 16 in humans (Visse \& Nagase 2003) and chromosome 8 in mice (Becker-Follmann et al. 1997). MMP2 can break down both collagen and elastin in the vessel walls (Keeling et al. 2005), and is able to bind to collagen and gelatin due to an insert of three repeats of a type III fibronectin domain into its catalytic domain (Allan et al. 1995).

It has been suggested that MMP2 is involved in the NE and ANGII induced inward remodeling in rat cremaster arterioles (Martinez-Lemus et al. 2011). Further, Chesler et al. (1999) suggest that MMP2 and MMP9 (discussed below) are both involved in the early remodeling associated with hypertension in porcine arteries (Chesler et al. 1999). Interestingly, MMP2 can induce vasoconstriction by cleaving big endothelin-1 which is only able to produce vasoconstriction in its cleaved form (Fernandez-Patron et al. 1999), and has been shown to induce inward remodeling in isolated arterioles (Bakker et al., 2004). Higher levels of serum MMP2 and MMP9 in humans results in an increased risk for heart failure (Collier et al. 2011).

In children, increased circulating levels of MMP2 were associated with an increase in SBP (Sesso \& Franco 2010). MMP2 is also elevated in individuals with hypertension and end-stage renal disease (Friese et al. 2009). Bergman et al. (2007) demonstrated a pathophysiological role of Mmp2 in the heart of mice (Bergman et al. 2007). By creating a cardiac-specific active Mmp2 with a mutation in the pro-domain, they showed that Mmp2 can lead to systolic dysfunction and left ventricular remodeling. 


\section{Matrix Metalloproteinase 9}

MMP9 is a 92kDa gelatinase, also known as gelatinase B. Activation of MMP9 occurs when its secreted zymogen form is cleaved to the $82 \mathrm{kDa}$ active form by peptidases, including other MMPs (Wilhelm et al. 1989). The gene encoding MMP9 is located on chromosome 20 in humans (Visse \& Nagase 2003) and 2 in mice (Leco et al. 1997). MMP9 has a similar structure and function to MMP2, but it cannot digest type I , II or III collagens (Visse \& Nagase 2003).

MMP9 is associated with outward remodeling in response to high intraluminal pressure (Lehoux et al. 2004) which is proposed to be a compensatory mechanism in order to increase vascular compliance (Martinez-Lemus \& Galinanes 2011). However, aneurysms can result from distension of the blood vessel if the upregulation of MMP9 is chronic (Keeling et al. 2005). This is demonstrated in a mouse model where knocking out Mmp9 made the mice resistant to the formation of aneurysms (Longo et al. 2002). Also, it has been shown in humans that the active concentration of MMP9 in abdominal aortic aneurysm is positively associated with the thickness of the aneurysm (Khan et al. 2012).

Increased plasma MMP9 in children has been shown to be a predictor of vascular dysfunction (Sesso \& Franco 2010). It has also been shown that higher plasma levels of MMP9 are associated with increased SBP (Friese et al. 2009). Increased blood pressure with MMP9 could be due to decreased vasodilation, as it has been demonstrated by Rodrigues et al. (2010) that injections of MMP9 and/or MMP7 (discussed below) into mesenteric venules in rats resulted in decreased arteriolar diameter (Rodrigues et al. 
2010). A reduced arteriolar diameter in response to MMP9 seems contradictory because MMP9 has been associated with outward remodeling (Lehoux et al. 2004), but Rodrigues et al. (2010) propose that cleavage of the $\beta 2$ adrenergic receptor by MMP9 and MMP7 in their study is responsible for the reduced vasodilation and arteriolar diameter (Rodrigues et al. 2010).

\section{Matrix Metalloproteinase 7}

MMP7 is 28kDa in its pro-enzyme form, and is cleaved to a $19 \mathrm{kDa}$ active form, and it is also known as matrilysin (Crabbe et al. 1992). MMP7 is the smallest MMP because it lacks a hinge region and $C$-terminal hemopexin-like domain (Gaire et al. 1994). This enzyme was first discovered in the postpartum rat uterus in 1980 (Sellers \& Woessner 1980). The gene encoding MMP7 is located on chromosome 11 in humans (Visse \& Nagase 2003) and on chromosome 9 in mice (Yamazaki et al. 1998).

In humans, elevated serum MMP7 has been associated with an increased risk of CVD (Tuomainen et al. 2012). Knockdown of Mmp7 in a model of vasoconstrictorinduced hypertension in spontaneously hypertensive rats stops the development of cardiac hypertrophy and reduces the severity of hypertension (Wang et al. 2009). It was later shown that Mmp7 is involved in upregulation of MMP2 in ANGII-induced hypertension in mice (Odenbach et al. 2011). In that study, MMP2 had a reduced ability to increase blood pressure when MMP7 was knocked down using a small interfering RNA. MMP7 has also been implicated in the development of atherosclerotic plaques by increasing vascular smooth muscle cell apoptosis (Williams et al. 2010). 


\section{c) Tissue Inhibitors of Matrix Metalloproteinases}

The tissue inhibitors of MMPs (TIMPs) regulate the activities of the MMPs endogenously. Four TIMPs have been described in humans [i.e. TIMP 1-4; reviewed in (Nagase \& Woessner 1999)]. In the TIMPs, disulfide bonds between the first cysteine and the $70^{\text {th }}$ cysteine residues are critical for the inhibition of the MMPs by allowing binding of the TIMPs to the active site in the MMPs (Gomis-Ruth et al. 1997). TIMPs have low selectivity for the inhibition of the MMPs, and they inhibit the MMPs in a 1:1 ratio (Willenbrock \& Murphy 1994). All TIMPs contain 12 cysteine residues that form six stable loops held together by disulphide bonds. The first three loops and the last three loops are found in the $\mathrm{N}$ - and $\mathrm{C}$ - terminal subdomains, respectively. The $\mathrm{N}$ - terminus is primarily responsible for the interaction between the TIMPs and the MMPs (Willenbrock \& Murphy 1994). The size of the TIMPs ranges from 21 to $29 \mathrm{kDa}$ (Murphy et al. 1991). While there are four TIMPs, I will focus on TIMP1 in this review because it is the one we used in our research.

\section{Tissue Inhibitor of Matrix Metalloproteinase 1}

TIMP1 can endogenously inhibit all of the MMPs aside from MMP14 (Will et al. 1996). The gene for TIMP1 is found on the $X$ chromosome in humans (Anderson \& Brown 2005) and in mice (Isensee et al. 2008). It has been shown that TIMP1 can form a complex with pro-MMP9 and control the rate of MMP9 activation (Itoh \& Nagase 1995).

TIMP1 is elevated in the serum of people with an increased risk of CVD (Tuomainen et al. 2012). Infusion of Timp1 in rat aortas has been shown to block the development of aortic aneurysms (Allaire et al. 1998). Timp1 knockout mice also 
develop larger aneurysms than control mice after aortic elastase infusion (Eskandari et al. 2005).

TIMP1 also has increased expression in Takayasu's arteritis (TA), an inflammatory disease found primarily in the aorta (Mahajan et al. 2012). Mice fed a high fat, high sugar "Western” diet had increased liver inflammation and expression of Timp1 (Ishimoto et al. 2013). The balance between TIMPs and MMPs is controlled in physiological conditions, but the balance can be disrupted in pathophysiological conditions like inflammation and tumor progression [Reviewed in (Gomez et al. 1997)].

\section{d) Reactive Oxygen Species and NADPH Oxidase}

One way that MMP activity can be increased is by increased levels of ROS (Martinez-Lemus et al. 2011). Oxidative stress associated with increased levels of ROS can occur during pathophysiological conditions like CVD [reviewed extensively by (Montezano \& Touyz 2013)]. As discussed below, NADPH Oxidase (NOX) derived ROS could provide a mechanism for the association between oxidative stress and CVD.

\section{Reactive Oxygen Species}

ROS are small molecules derived from oxygen that act as oxidizing agents such as superoxide $\left(\mathrm{O}_{2}{ }^{-}\right)$, peroxyl and hydroxyl radicals, and hydrogen peroxide $\left[\mathrm{H}_{2} \mathrm{O}_{2}\right.$; (Bedard \& Krause 2007)]. $\mathrm{O}_{2}^{-}$generation, where oxygen accepts one electron, is usually the first step in the production of ROS [Fig. 2.1; (Klebanoff 1980)]. $\mathrm{O}_{2}{ }^{-}$can then be converted to other radicals such as $\mathrm{H}_{2} \mathrm{O}_{2}$ by superoxide dismutase [Fig. 2.1; (Li et al. 2001)]. While ROS are important for physiological processes like cell migration and 
proliferation, an over production of ROS can result in adverse outcomes like cell death, cancer, diabetes and CVD (Igosheva et al. 2010, Garrido-Urbani et al. 2011). NOX, which is the main enzyme responsible for ROS production in the vasculature will be discussed below, as well as how ROS produced by NOX have been associated with CVD.

\section{NADPH Oxidase}

NOX was first discovered as part of a respiratory burst in cells that had been described early in the $20^{\text {th }}$ century (Baldridge 1932, MacLeod 1943), and it was then discovered that NOX was responsible for the production of $\mathrm{O}_{2}{ }^{-}$and $\mathrm{H}_{2} \mathrm{O}_{2}$ during this event (Rossi \& Zatti 1964, Babior et al. 1973). NOX family members on the membrane of the mitochondria reduce oxygen into $\mathrm{O}_{2}{ }^{-}$by helping transport electrons across the membrane (Garrido-Urbani et al. 2011). All NOX enzymes have an NADPH binding site, a FAD-binding region, six transmembrane domains and four heme-binding histidines (Bedard \& Krause 2007). FAD is involved in the regulation of electron transfer by NOX (Hashida et al. 2004).

NOX is composed of several subunits that act as an enzyme complex to produce $\mathrm{O}_{2}{ }^{-}$, which can then be converted to other forms of ROS (Bedard \& Krause 2007). The first NOX discovered was gp91 ${ }^{\text {phox }}$ [NOX2; (Royer-Pokora et al. 1986)], and several isoforms have been discovered since. The NOX family is now known to include seven isoforms with two subunits that contribute to organization of the complex ( $\mathrm{p} 47^{\mathrm{phox}}$ and NOXO1), two subunits that help activate the complex (p67 $7^{\text {phox }}$ and NOXA1) and 


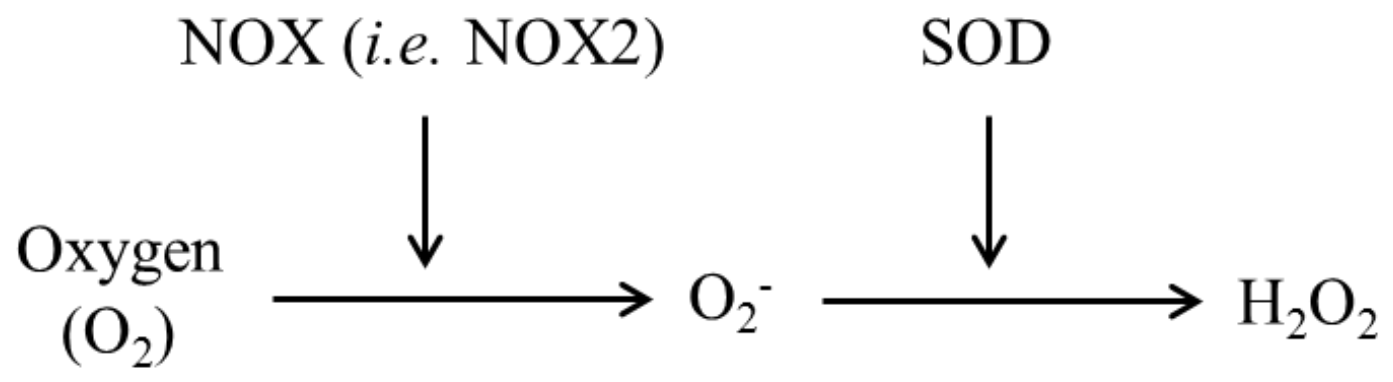

Figure 2.1 Production of superoxide and hydrogen peroxide from oxygen. The donation of an electron by NOX to turn $\mathrm{O}_{2}$ into $\mathrm{O}_{2}{ }^{-}$is usually the first step in ROS production. $\mathrm{O}_{2}{ }^{-}$ can then be converted to another ROS $\mathrm{H}_{2} \mathrm{O}_{2}$ by SOD. NOX= NADPH Oxidase. $\mathrm{O}_{2}{ }^{-}=$ superoxide. $\mathrm{SOD}=$ superoxide dismutase. $\mathrm{H}_{2} \mathrm{O}_{2}=$ hydrogen peroxide. 
maturation factors for large members of the NOX family [DUOXA1 and DUOXA2; (Bedard \& Krause 2007)]. NOX is the main producer of ROS in the vascular wall (Cai et al. 2003).

\section{NADPH Oxidase 2}

NOX2, also known as CYBB and gp91 ${ }^{\text {phox }}$, is a membrane-bound component of NADPH oxidase and is encoded by a gene located on the $\mathrm{X}$-chromosome in humans (Bedard \& Krause 2007) and mice (Chandra et al. 2011). NOX2 is heavily glycosylated (Harper et al. 1985). Glycosylation of NOX2 has been shown to increase the production of ROS (Cai et al. 2010).

The mechanism for NOX2 activation has been described by Bedard and Krause (Bedard \& Krause 2007). NOX2 forms a complex with cytochrome b-245 light chain $\left(\mathrm{p} 22^{\text {phox }}\right)$, which is translocated to the membrane by phosphorylated $\mathrm{p} 47^{\text {phox }}$. This brings $\mathrm{p} 40^{\text {phox }}$ (a small subunit) into the complex allows the "activator subunit” $\mathrm{p} 67^{\text {phox }}$ to interact with NOX2. The GTPase Rac then interacts with NOX2 followed by interaction with $\mathrm{p} 67^{\mathrm{phox}}$, and completes the assembly and activation of the complex (Bedard \& Krause 2007).

Nox2 gene expression can be regulated by the promoter region (Skalnik et al. 1991), and also by upstream repressing factors and activating factors such as nuclear factor kappa-light-chain-enhancer of activated B cells [NFkB; (Anrather et al. 2006)]. NOX2 de novo protein synthesis can also be induced in resistance arteries by ANG-II (Touyz et al. 2002). $\mathrm{H}_{2} \mathrm{O}_{2}$, which is a downstream product from $\mathrm{O}_{2}{ }^{-}$degradation, can act 
in a feed-forward mechanism by further increasing the production of $\mathrm{O}_{2}{ }^{-}$by NOX2 ( $\mathrm{Li}$ et al. 2001).

A role for NOX2 in several CVD states has been demonstrated in humans and in rodents. Nox2 is elevated in myxomatous mitral valve disease, a disease associated with valve thickening and matrix remodeling in mice (Hagler et al. 2013). NOX2 expression is also higher in aortic abdominal aneurysms in humans (Guzik et al. 2013). Women who are deficient in NOX2 display less vascular damage and atherosclerosis (Violi et al. 2013).

Sukumar et al. (2013) demonstrated in a mouse model that knocking down Nox2 reduced $\mathrm{O}_{2}{ }^{-}$levels and improved vascular function in insulin-resistant endothelial cells (Sukumar et al. 2013). Another study revealed that mice without Nox2 had less vascular dysfunction and reduced $\mathrm{O}_{2}^{-}$levels after induced high pressure in cerebral arterioles when compared to wild-type mice (Chan \& Baumbach 2013). Also, mice lacking Nox2 develop ANGII-induced hypertension more slowly than those that have wild-type Nox2 (Haque \& Majid 2011).

\section{e) Relationship between ROS and MMPs}

Increased oxidation due to higher levels of ROS can activate the MMPs by the inhibition of the nuclear hormone receptor peroxisome proliferator-responsive element $\alpha$ [PPAR $\alpha$; (Garrido-Urbani et al. 2011)]. PPAR $\alpha$ normally acts in an anti-angiogenic manner by inhibiting the NFkB pathway, involved in producing more MMPs. Therefore, when PPAR $\alpha$ is inhibited by ROS more MMPs are able to be produced through the NFkB pathway, allowing for more remodeling (Garrido-Urbani et al. 2011). NFkB is also an 
activating factor that induces greater production of ROS by NOX (Anrather et al. 2006). PPAR $\alpha$ has also been shown to be downregulated with ANGII stimulation, resulting in increased expression of inflammatory cytokines in rat vascular smooth muscle cells (Ji et al. 2009).

Oxidative stress can increase the activity of several MMPs including MMP2 and MMP9 in both neonatal and adult cardiac fibroblasts (Siwik et al. 2001). Increased ROS can also increase the expression of MMPs in vascular smooth muscle cells (Zhang et al. 2013). In rat embryonic heart cells, increased NOX-derived ROS results in increased expression of Mmp9 (Yang et al. 2013). In a model of NE and ANGII stimulation in rat cremaster arteries, $\mathrm{O}_{2}{ }^{-}$was the ROS responsible for activating MMPs in the inward remodeling process (Martinez-Lemus et al. 2011). It was also shown that when ROS were inhibited during NE and ANGII stimulation, the inward remodeling did not occur. This shows that ROS are required for the inward remodeling induced by NE and ANGII (Martinez-Lemus et al. 2011). Mechanical stretch in vasculature which is associated with hypertension also increases Mmp2 expression due to an increase in NOX-derived ROS (Grote et al. 2003).

When increased ROS interact with NO, they form a molecule called peroxynitrite which has been shown to inactivate TIMP1 (Frears et al. 1996) and activate MMPs in smooth muscle cells (Rajagopalan et al. 1996). Peroxynitrite can also activate MMP1, 8 and 9 at a posttranslational level; however, this action does not involve removing the MMP pro-domain (Okamoto et al. 2001). 


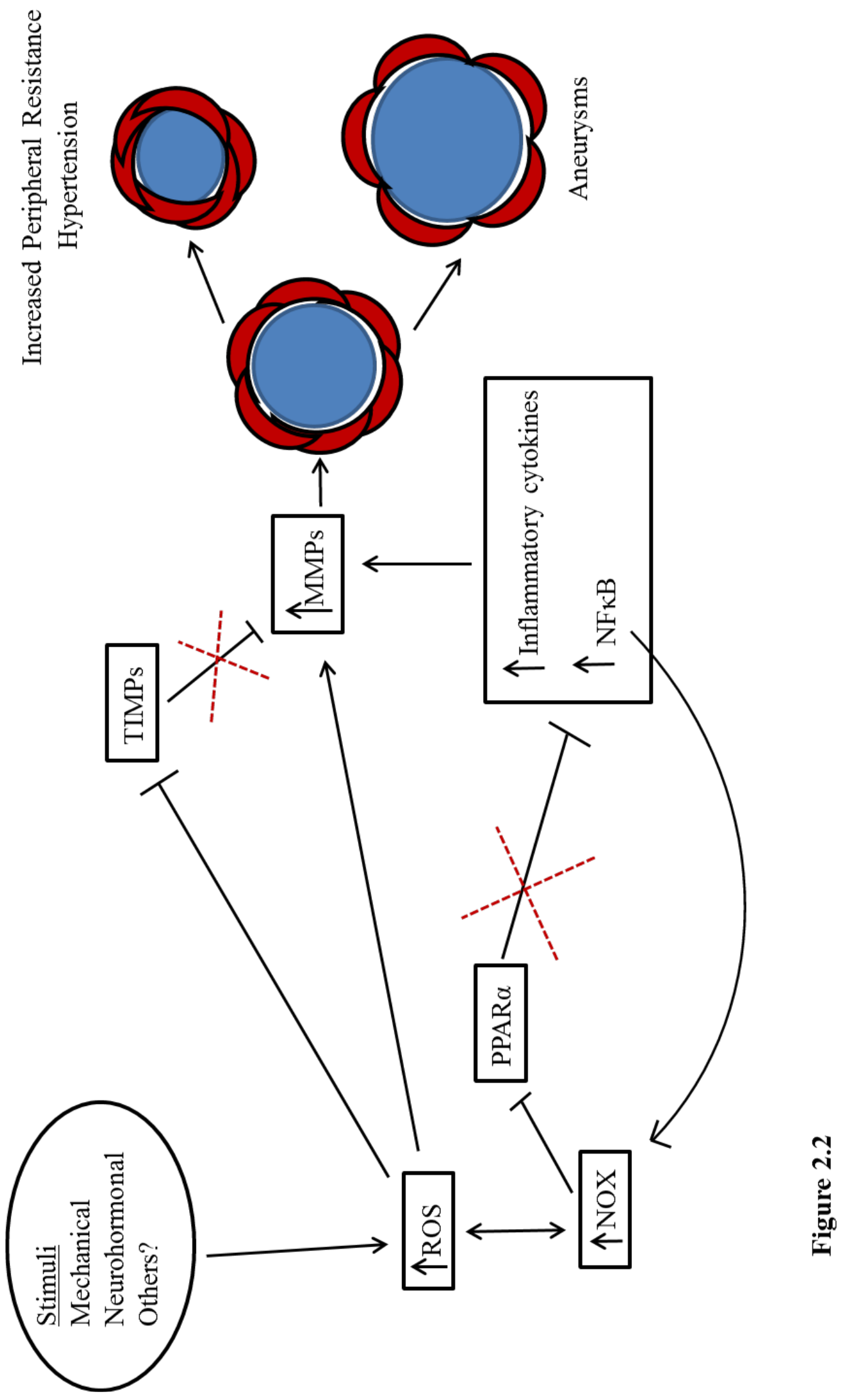


Figure 2.2 Involvement of MMPs, TIMPs, NOX and ROS in vascular remodeling. Stimuli such as increased angiotensin II (ANGII, neurohormonal) or increased vascular stretch (mechanical) can result in increased production of reactive oxygen species (ROS) through NADPH oxidase (NOX). Increased ROS can increase the expression and activity of matrix metalloproteinases (MMPs) and decrease the activity of their endogenous inhibitors tissue inhibitors of matrix metalloproteinases (TIMPs). Increased NOX results in inhibition of the nuclear receptor PPAR- $\alpha$, thereby increasing inflammatory cytokines and NFאB due to decreased inhibitory activities of PPAR- $\alpha$. The NFkB pathway and inflammatory cytokines can both increase the activity of MMPs. $\mathrm{NF \kappa B}$ can also cause an elevated increase in ROS, further perpetuating the pathway. Increased activity and expression of MMPs results in degradation of the ECM allowing repositioning of vascular smooth muscle cells in inward and outward remodeling associated with adverse outcomes such as hypertension and aneurysms, respectively. Other stimuli that can result in increased ROS will be discussed further in this review. Red x's represent a decrease in inhibition by PPAR $\alpha$ and TIMPs. 


\section{f) Conclusion}

This section has shown how the MMPs can be involved in the development of CVD. Along with adverse consequences on cardiovascular health, MMPs and ROS have also been proposed to be involved in the inward eutrophic remodeling associated with the development of hypertension (Martinez-Lemus \& Galinanes 2011). A summary of some ways that the NOX-produced ROS, MMPs and TIMPs can interact to affect vascular remodeling and cardiovascular health is represented in Fig. 2.2.

\subsection{Developmental Origins of Health and Disease Hypothesis}

\section{a) Introduction to the DOHaD Hypothesis}

The idea that the in utero environment can affect the development of disease in adulthood became aptly known as the Fetal Origins of Adult Disease Hypothesis [FOAD; Reviewed in (Calkins \& Devaskar 2011, Poston 2011)]. Dr. David Barker discovered this phenomenon in retrospective human studies, and the hypothesis is now commonly being tested in animals and humans to shed light on what suboptimal maternal environments could be contributing to the widespread occurrences of obesity and CVD.

Barker's studies followed the initial observation by Forsdahl that poor nutrition in childhood followed by overnutrition as an adult in a Norwegian population resulted in an increased risk of coronary heart disease (Forsdahl 1977). The FOAD hypothesis has

now been termed the Developmental Origins of Health and Disease hypothesis (DOHaD) to encompass the effects that can happen prior to the fetal stage [i.e. the oocyte and early 
embryo; (Gillman et al. 2007)]. This section will discuss observations found in studies regarding maternal undernutrition and its effects on the offspring. Maternal undernutrition is a topic that has been extensively studied since the initial observations that famine exposure during pregnancy resulted in adverse outcomes in the children (Gilbert et al. 2005, Watkins et al. 2008, Lan et al. 2013, Tchoukalova et al. 2013).

\section{b) Barker’s Cardiovascular Studies}

Dr. David Barker and colleagues have performed numerous retrospective studies regarding maternal exposure to famine and its effects on the future disease state of their children as adults. These studies stemmed from the initial observation that sharp increases in the occurrence of CVD correlated not with areas that were known for consuming an unhealthy diet, but rather with areas that had experienced high levels of past infant mortality associated with low birth weight (Barker \& Osmond 1986). Low birth weight has since been associated with adverse outcomes including increased risks for coronary heart disease, hypertension and stroke (Barker et al. 1989, Eriksson et al. 2000, Painter et al. 2006a). Further studies have also revealed associations between experiencing famine in utero and the subsequent development of CVD, Type II Diabetes Mellitus (T2DM), obesity, stroke and psychological disorders (Barker et al. 1989, Hales et al. 1991, Brown 2000, Lussana et al. 2008). A sample of findings from three of the cohort studies commonly used to retrospectively study the effects of low birth weight, early development and maternal undernutrition in humans are discussed below. 


\section{Hertfordshire Cohort Study}

Midwives in the Hertfordshire area began recording birth weights at the births they attended in 1911, and other health officials visited the homes throughout infancy to perform health records and to measure the weight at one year of age (Barker et al. 2009). It was discovered that men and women who had lower birth weights had an increased risk of dying from coronary heart disease (Barker et al. 1989, Osmond 1993). A follow-up study in males who had low birth weights showed that they acquired higher cholesterol levels with the consumption of high fat foods, which can result in an increased risk for coronary heart disease (Robinson 2006). Males from the Hertfordshire Cohort also had greater risk for impaired glucose tolerance and T2DM if they had low birth weight (Hales et al. 1991).

\section{Helsinki Cohort Studies}

Birth records were also well-recorded in Helsinki from 1934-1944 and followed 13,345 men and women (Eriksson et al. 2001, Osmond et al. 2007). The results from this birth cohort have been used in many diverse studies that have examined topics ranging from cardiovascular development to obesity and psychological outcomes. An association between low birth weight and increased adiposity and serum triglycerides was made from this cohort (Kajantie et al. 2008). Another study found that increased childhood body mass index (BMI) resulted in less cardio respiratory fitness in adulthood (Salonen et al. 2011).

Yet another study found that increased CVD death in females was associated with low length at birth and low birth weight in females and males, respectively (Kajantie et al. 2005). Interestingly, fetal growth restriction; specifically, a lower birth weight with 
a higher head circumference, increased the risk for coronary heart disease (Eriksson et al. 2001). Similarly, results were obtained in an earlier Helsinki birth cohort study (from 1924 to 1933) where men who had low birth weight were shown to have an increased risk for stroke as adults (Eriksson et al. 2000). The placenta is involved in nutrient transfer to the growing fetus, and a thin placenta—associated with shallow invasion of the spiral arteries and reduced nutrient transfer to the fetus—-has been shown to increase risk for sudden cardiac death in men and women in the Helsinki Cohort Studies (Barker et al. 2012).

\section{Dutch Famine}

Low birth weight can result from decreased nutrient availability or decreased nutrient transfer from the mother (Harding 2001). With the observations that low birth weight could affect the development of the offspring, studies of maternal exposure to famine became of interest. The Dutch famine in the 1940's is an example of a time when maternal nutrition was shown to affect the development of offspring [(Tobi et al. 2009), reviewed in (Calkins \& Devaskar 2011)]. The famine occurred over a short period of time in 1944 and 1945, but detailed records of people born in Amsterdam during this time period were kept, allowing for studies to be performed in this population (Carroll et al. 2012). The short length of the famine in a previously well-nourished population allows for determination of undernutrition effects at specific time points in pregnancy (Roseboom et al. 2006). One of the first observations from the children born during the famine was that they had lower birth weights (Smith 1947).

Exposure to famine in utero can lead to adverse metabolic outcomes in the offspring. For example, in the Dutch famine 50-year-old women who had experienced 
famine in utero during the first 16 weeks of gestation tended to have a higher BMI (Ravelli et al. 1999), and children of women who experienced the famine during the last 32 weeks of pregnancy tended to have a lower glucose tolerance (Ravelli et al. 1998) than those that did not experience famine. Lipid profiles of children exposed to famine in utero have been shown to be increased (Lussana et al. 2008), and an effect on increased adiposity persisted into the next generation (Painter et al. 2008).

Other studies have found links between in utero exposure to the Dutch famine and adverse psychological outcomes including Major Affective Disorder and Schizophrenia (Brown 2000, Brown \& Susser 2008). Women exposed to famine in utero also had increased risk for breast cancer; especially when they were exposed during early gestation (Painter et al. 2006c, van Abeelen et al. 2012).

The cardiovascular system has also been shown to be affected by famine exposure in utero. Increased resting blood pressure was most often observed when the mothers had consumed a small ratio of protein to carbohydrates, rather than a low macronutrient level overall (Roseboom 2001). Famine during late gestation resulted in decreased vessel lumen diameter in the offspring (Painter et al. 2007), while exposure to famine in utero during early gestation increased the risk for coronary artery disease in individuals in their 50's, and the average age at which these individuals experienced coronary artery disease was 47 years of age as opposed to 50 years of age in individuals who had not been exposed to famine (Painter et al. 2006b). In addition, people born to mothers who experienced famine during early pregnancy exhibited higher blood pressure increases during psychological stressors such as public speaking (Painter et al. 2006a). Higher 
increases in SBP in response to stress has been associated with future development of hypertension (Carroll et al. 2012).

\section{c) Low Birth Weight, Developmental Plasticity and the "Thrifty Phenotype”}

The nutritional environment while in utero is very important in determining the subsequent development of the offspring. This is due to reduced availability of nutrients in utero (Harding 2001). In a retrospective study used to examine the effect of genetics versus the effect of in utero environment on the birth weight of babies in cases of oocyte donation, the size of the baby was determined by the size of the mother who developed the fetus in utero rather than by the size of the mother who donated the oocyte (Brooks et al. 1995). The DOHaD hypothesis (mentioned earlier) predicts that the maternal environment a fetus is exposed to can cause it to have a higher incidence of certain diseases later in life [Reviewed in (Calkins \& Devaskar 2011, Poston 2011)]. This is due to a concept called “developmental plasticity,” which refers to multiple phenotypes resulting from one genotype in response to different environmental cues [reviewed in (Calkins \& Devaskar 2011), Jirtle and Skinner, 2007)].

Programming occurs when the phenotypic changes acquired in response to a specific environment become permanent. This can be beneficial when the subsequent environment is similar to the in utero environment and the programmed phenotype allows the individual to perform well in the subsequent environment [reviewed in (Brenseke et al. 2013)]. However, the changed phenotype can become maladaptive if the subsequent environment does not match the original environment to which the individual was programmed. For example, an individual who experiences low nutrition in utero can 
develop a "thrifty phenotype” where the development of organs like the pancreas and kidney are stunted in favor of developing vital organs like the brain, which can then predispose the individual to diseases like diabetes and hypertension when the individual has high nutrient availability later in life [(Hales 1992) reviewed in (Brenseke et al. 2013)].

\section{d) Animal Models of Undernutrition}

Due to a limited number of detailed birth records in humans, animal models are often used to study the effects of maternal undernutrition on offspring development. One commonly-used model of animal undernutrition is that in which a low protein diet is given to the mother. A maternal low protein diet supplied only during oocyte development in mice had adverse outcomes on the offspring including reduced arterial function in males and increased SBP in males and females (Watkins et al. 2008). A study in sheep also revealed that a diet with low amino acid content resulted in decreased methylation of two imprinted genes in the offspring when compared to those exposed to higher amino acid content in utero (Lan et al. 2013). Abnormal methylation of imprinted genes can affect the ability of the offspring to develop, as proper imprinted gene expression is essential for the development of the fetus and placenta (Fowden et al. 2006).

A study of low protein diet in mice by Watkins et al. (2011) revealed adverse effects on the cardiovascular and metabolic health of the offspring. Adverse effects were observed in that study regardless of whether the diet was provided while the oocyte was developing, during preimplantation development, or throughout the entire gestation. One 
effect shown in the study was increased SBP at one year of age in all of the low protein diet groups. Also, maternal consumption of a low protein diet during gestation resulted in decreased female offspring body weight compared to controls, and low protein diet during embryo development resulted in female offspring with increased body weight (Watkins et al. 2011). It has also been observed in rats that females exposed to low protein diet in utero show a preference for high fat foods, suggesting a possible link between maternal consumption of a low protein diet and diet-induced obesity in the offspring (Bellinger et al. 2004). Amino acids in protein help with antioxidant synthesis, so decreased availability of amino acids can lead to greater oxidative damage [reviewed in (Brenseke et al. 2013)].

While protein deficiency is a common animal model of undernutrition, diets with global nutrient restriction (Gilbert et al. 2005, Tchoukalova et al. 2013), methyldeficiency (Maloney et al. 2011), and low polyunsaturated fatty acids [PUFA's;(Armitage et al. 2003, Mathai et al. 2004)] have also been used to represent maternal undernutrition. An effect of greater oxidative damage on offspring development has been demonstrated in a model of maternal nutrient restriction in rats (Franco Mdo et al. 2004). When male rats from undernourished mothers were supplemented with an essential cofactor for NO, they had less ROS production and improved endothelial function, suggesting that oxidative damage is involved in the development of endothelial dysfunction with maternal undernutrition (Franco Mdo et al. 2004).

In baboons, a thirty percent reduction in global nutrients resulted in decreased body weight and increased expression of adipogenesis-related genes in male fetuses (Tchoukalova et al. 2013). Global maternal nutrient restriction in sheep increased MAP 
in the lambs and increased ACE expression (Gilbert et al. 2005). A methyl-donor deficient maternal diet in rats resulted in male offspring with higher glucose levels and insulin resistance at 8 months of age when compared to controls (Maloney et al. 2011). When female rats consumed a PUFA-deficient diet, the offspring displayed increased MAP (Armitage et al. 2003) as well as increased appetite signaling (Mathai et al. 2004) as adults.

In summary, the initial observation that low birth weight was associated with later disease state led to interest in studying the effect that maternal nutrition has on the health of the offspring. Records from several human birth cohorts have provided insight on low birth weight, and the records from the Dutch Famine provide a glimpse on the effects of maternal undernutrition in humans. However, animal studies of maternal undernutrition are used to investigate the effects that certain dietary deficiencies, including low protein diets and methyl donor deficiencies have on the development of the offspring. Together, these studies have provided the initial insight that the environment in which a fetus develops can affect the disease state of the offspring in adulthood. Due to the increasing epidemic of obesity (Kelly et al. 2008), the DOHaD hypothesis has been expanded to include the effects of maternal obesity and overweight (Armitage et al. 2008). This will be discussed in the following section. 


\subsection{Obesity}

\section{a) Prevalence}

It is estimated that by 2030 almost sixty percent of adults world-wide will be overweight or obese. Body mass index; (weight in $\mathrm{kg}$ divided by height in $\mathrm{m}^{2}$ ) is used as a measure of overweight and obesity. Overweight is defined as a BMI greater than twenty-five and obesity is a BMI greater than thirty in adults. The rate of obesity is highest in developed and developing countries (Kelly et al. 2008). Over 200 million men and 300 million women world-wide were considered obese in 2008 (WHO 2013e). Twothirds of adults in the United States are overweight and one-third are obese (NIH 2012). Also, 64\% of women in the United States are either overweight or obese (NIH 2012).

Obesity has also become an increasing problem in children. World Health Organization (WHO) statistics showed that globally there were over forty-two million children under the age of five who were overweight in 2010 (WHO 2013c). For children, overweight is at or above the 85th percentile, while obesity is at or above the 95th percentile of body weight (NIH 2012). Obesity is associated with increased risk for other health problems including hypertension, certain cancers, and T2DM (NIH 2012).

\section{b) Effect of Obesity and Overweight on Oocyte Competence}

Maternal obesity and overweight can have adverse effects on female reproductive function by its effects on the oocyte (Igosheva et al. 2010, Luzzo et al. 2012, Machtinger et al. 2012). In humans and in mice, using oocytes from a non-obese female for in vitro fertilization (IVF) and embryo transfer into an obese female resulted in less adverse developmental effects than if the oocyte was from an obese female (Luke et al. 2011a, 
Luzzo et al. 2012). This suggests that poor oocyte quality is predictive of adverse outcomes in utero. An inflammatory environment induced by obesity has been observed in the ovaries of obese women, providing an altered environment for oocyte development (Robker et al. 2009).

A pro-inflammatory environment can result in increased oxidative stress by the production of ROS [Reviewed in (Fernandez-Sanchez et al. 2011)]. A mouse model of maternal obesity demonstrated that oocytes from obese dams had abnormal mitochondrial distribution and function, and that this resulted in increased production of ROS (Igosheva et al. 2010). Increased levels of ROS can affect oocyte developmental potential (Jiao et al. 2013). In addition, oocytes from obese women are more likely to have abnormal meiotic spindles and misaligned chromosomes during the metaphase II (MII) phase of meiosis than oocytes from women with normal BMI’s [18.5-24.9; (Machtinger et al. 2012)]. Also, higher levels of nonesterified fatty acids (NEFA) that can be found in obesity have been shown to upregulate genes involved in energy metabolism and oxidative stress in bovine oocytes (Van Hoeck et al. 2013).

\section{c) Effect of Obesity and Overweight on Preimplantation Embryo Development}

The pro-inflammatory environment in obesity can affect the uterus. The uteri from obese dams in a rat maternal obesity model had increased expression of proinflammatory genes at day 4.5 post-coitum (Shankar et al. 2011). Shankar et al. (2011) also showed that male preimplantation embryos had increased expression of genes involved in pro-inflammatory immune response and decreased expression of antiapoptotic genes (Shankar et al. 2011). 
Embryo development can also be affected by maternal obesity. It has been shown that embryos from obese females are more likely to have delayed development, chromosomal abnormalities and are more likely to be degraded than those from their nonobese counterparts (Binder et al. 2012, Luzzo et al. 2012). Further, Van Hoeck et al. (2011) showed that bovine embryos exposed to high NEFA during maturation had reduced viability as demonstrated by reduced oxidative metabolism and increased apoptosis in day 7 blastocysts (Van Hoeck et al. 2011).

\section{d) Adverse Effects of Obesity on Post-implantation Development}

The abnormalities during preimplantation development associated with an environment surrounded by adipose tissue can affect the development of the fetus in utero. The obese environment in humans has been associated with adverse outcomes during pregnancy including pre-eclampsia, intrauterine death, neural tube defects, gestational diabetes mellitus, the need for emergency C-sections, as well as a risk for having children born as large $\left(>90^{\text {th }}\right.$ percentile in weight) for gestational age [LGA;(Sebire et al. 2001, Di Cianni et al. 2005, Chu et al. 2007, Rasmussen et al. 2008)]. The effects of low birth weight have been previously described, but high birth weight can also have adverse effects on a person's future disease state, including increased risk for obesity (Sorensen et al. 1997). However, in mouse models, maternal obesity is associated with decreased in utero development and lower birth weight in the offspring (Luzzo et al. 2012). Although mice offspring from mothers exposed to excess fatty acids tend to be born small, they catch up and quickly surpass the size of mice born to mothers with normal fatty acid levels (Jungheim et al. 2011). 
The placenta is responsible for transporting nutrients to the growing fetus. The placenta has been shown to be affected by maternal obesity (Liang et al. 2010, Hayes et al. 2012). In mice, diet-induced obesity resulted in oxidative stress in the placenta, adversely affected trophoblast invasion, and resulted in more cellular necrosis in the placenta (Liang et al. 2010). Obese dams in a rat model had increased rates of fetal death, most likely caused by the altered vasculature found in the placentas of these dams (Hayes et al. 2012).

These in utero effects can also affect the future disease state of the offspring. Children born to obese mothers are more likely to develop metabolic syndrome (MS) with symptoms including high blood pressure, lowered glucose tolerance and increased body weight (Boney et al. 2005). Women who are obese also have offspring with higher incidences of obesity (Nelson et al. 2010, Poston 2011, Morandi et al. 2012). Also, in a prospective study in women in Australia, maternal high BMI was associated with offspring high BMI, and this association was more pronounced than the association between paternal BMI and offspring BMI (Lawlor et al. 2007).

Rodent models of maternal obesity have revealed increased body weight in the offspring (Samuelsson et al. 2008, Franco et al. 2012) and impaired development of skeletal muscles (Bayol et al. 2005). A study in mice found increased body weight and body fat mass in thirty-week-old male offspring born to obese mothers even though the offspring consumed a normal diet after weaning (Torrens et al. 2012). Males from obese dams also have been shown to exhibit glucose intolerance (impaired glucose tolerance) and increased inflammatory adipokines, which are cytokines released by adipose tissue (Magliano et al. 2013). Hyperleptinemia and hyperinsulinemia have also been observed 
in offspring from rodent maternal obesity models (Shankar et al. 2010, Franco et al. 2012). Hyperleptinemia can result in increased rates of obesity and increased blood pressure in the offspring as adults (Trevenzoli et al. 2007).

Maternal Obesity/Overweight and Cardiovascular Abnormalities in the Offspring

A maternal overweight or obese environment can also affect vascular functioning and development of hypertension in the offspring (Samuelsson et al. 2008, Samuelsson et al. 2010, Torrens et al. 2012). In mice, prenatal exposure to maternal obesity resulted in increased SBP, decreased NO production and decreased vasorelaxation to acetylcholine in the femoral arteries in male offspring at thirty weeks of age (Torrens et al. 2012). It was also found that the generation of NADPH-dependent $\mathrm{O}_{2}{ }^{-}$was increased in the liver of these males. Increased ROS have been associated with endothelial dysfunction (Chinen et al. 2007).

While not as many studies have been performed in humans as in animal models, associations have still been made between a maternal overweight and obesity and cardiovascular outcomes in the offspring. An Australian cohort study in five-year-old children revealed that maternal BMI was one of the factors that was associated with an increased SBP in the children (Lawlor et al. 2004). Another study found that an increase in maternal BMI was associated with increased leptin, SBP, and BMI in the children, regardless of whether or not the mother had diabetes (West et al. 2011).

\section{e) Maternal Consumption of a High Fat Diet}

In some studies the goal is not to examine the effect of maternal obesity, but rather to examine how a mother consuming a high fat (HF) diet during gestation and/or 
lactation can affect the offspring. A study in mice found that global DNA methylation of imprinted genes important for proper in utero development was lower in the placentas of female offspring when the mothers consumed a HF diet during pregnancy (GallouKabani et al. 2010). Maternal overnutrition during the last month of pregnancy in sheep affected fetal development as demonstrated by increased levels of glucose and insulin in fetal plasma, as well as increased expression of genes involved in adipogenesis (Muhlhausler et al. 2007).

High fat diet consumption during pregnancy can also affect the cardiovascular outcome in the offspring. Maternal consumption of a diet containing $21 \%(\mathrm{w} / \mathrm{w})$ fat during pregnancy and lactation in rats decreased the ability of the offspring aortas to relax in response to the potent vasodilator acetylcholine (Kelsall et al. 2012). ROS levels have also been shown to be increased in the hearts of mice offspring that were exposed to a HF diet both prenatally and postnatally (Turdi et al. 2013).

Increased maternal gestational weight gain which can occur as a result of HF diet consumption during pregnancy can also affect offspring development. In a retrospective cohort study in humans, children at seven years of age were more likely to be overweight if their mother had gained more than the recommended amount of weight during pregnancy, showing that diet during pregnancy is important (Wrotniak et al. 2008). Another study from an Australian birth cohort found that increases in maternal gestational weight gain tended to increase SBP in the children at 21 years of age (Mamun et al. 2009). 


\section{f) Effect of High Fructose Consumption}

High fructose consumption during pregnancy can also affect offspring development. High fructose corn syrup is a sweet corn based syrup made by using glucose isomerase to convert starch from corn into glucose, and then converting this glucose into fructose (Bray et al. 2004). Due to its sweet flavor, high fructose corn syrup is now often used to replace sugar in commonly consumed foods and drinks [i.e. baked goods and carbonated beverages; (Hanover \& White 1993)]. In approximately the past thirty years, the amount of fructose that is consumed by children in the United States has increased 10-20\% (Marriott et al. 2009).

Male rats born to mothers that consumed fructose in their drinking water during lactation had increased body weight, increased insulin and leptin levels, and consumed more food than control rats (Alzamendi et al. 2010). Another study found that rats born to mothers who had consumed 50\% fructose during pregnancy were hyperglycemic at birth (Jen et al. 1991). Higher fasting insulin levels (Rawana et al. 1993) and increased plasma leptin and glucose (Vickers et al. 2011) have also been observed in rats born to mothers that consumed fructose.

Fructose is sometimes included in obesity models to represent a "Western diet" where high levels of sugars are consumed. The consumption of fructose in adolescents increases cardiometabolic risk factors, and this is shown to be mediated by visceral adiposity (Pollock et al. 2012). Also, increased blood pressure and plasma insulin and triglycerides were shown in rats that consumed a high fructose diet for two weeks (Hwang et al. 1987). Another rat study showed that consuming a HF diet with fructose 
increased leptin in rats and increased SBP and endothelial dysfunction (Panchal et al. 2011).

\subsection{Assisted Reproductive Technologies}

\section{a) Introduction}

Infertility affects around 10\% of females in the United States (CDC 2013). To help overcome this infertility, couples may seek the use of assisted reproductive technologies (ART). In 2010, there were 61,564 infants born (slightly more than 1\% US births) that were conceived with the use of ART (CDC 2012). ART procedures include non-invasive techniques such as ovarian hyperstimulation and intrauterine insemination, embryo culture and IVF or invasive procedures such as intracytoplasmic sperm injection (ICSI). For clinics that are members of the Society for Assisted Reproductive Technologies (SART), there were 154,412 ART cycles performed in 2011 (SART 2013). The use of ART is increasing, with 107,587 cycles performed in 2001 and 147,260 in 2010 (CDC 2012).

\section{b) ART Procedures}

\section{Ovarian Hyperstimulation}

Ninety-nine percent of the cycles performed in 2011 in SART clinics used ovarian hyperstimulation (SART 2013). In ovarian hyperstimulation, exogenous gonadotropins are administered to increase the number of oocytes ovulated in a single reproductive (menstrual) cycle. This is done to provide a greater number of mature eggs to be used in 
the subsequent ART procedure (Zegers-Hochschild et al. 2009). The Center for Disease Control (CDC) does not consider ovarian hyperstimulation and ART procedure (CDC 2012), but we consider the use of exogenous gonadotropins ART in our laboratory because we have observed effects of the gonadotropins alone on gene expression and global DNA methylation in oocytes (Almamun 2011). In mice, ovarian hyperstimulation is known as superovulation (SO), and is commonly performed by administering five international units (IU) eCG followed by 5IU hCG approximately 48 hours later (Luo et al. 2011). The eCG has follicle stimulating hormone (FSH) activity, and regulates granulosa cell differentiation and proliferation, while the hCG has luteinizing hormone (LH) activity, and induces ovulation (Nagy A 2003).

In humans, ovarian stimulation is used not only to recruit multiple follicles to obtain more oocytes for the ART procedures, but also to remove the need to detect the LH surge in order to obtain the oocytes at the correct stage of maturity (Davis \& Rosenwaks 2001). Often gonadotropin releasing hormone (GnRH) agonists are used to create a surge in endogenous LH and FSH, and this surge results in the downregulation of the endogenous gonadotropins due to desensitization in the anterior pituitary (Davis \& Rosenwaks 2001). By downregulating endogenous gonadotropins, the exogenous gonadotropins determine the timing of the cycle. The ovarian follicles are stimulated daily by administration of FSH in the form of human menopausal gonadotropin (hMG), purified urinary FSH or recombinant FSH, starting at day 2 or 3 of the menstrual cycle. The hCG administration in humans is timed carefully to be performed when the mean size of the lead follicles is above $16 \mathrm{~mm}$ in diameter. The oocytes for the subsequent 
ART procedures are collected between 34 and 36 hours after hCG administration (Davis \& Rosenwaks 2001).

\section{IVF and ICSI}

In IVF, the embryo is formed by culturing the sperm and oocyte in fertilization medium and allowing the sperm to naturally fertilize the oocyte. IVF was used for 99\% of the ART cycles performed in 2010 (CDC 2012). Intracytoplasmic sperm injection (ICSI) is a specific type of IVF that involves manually injecting a single sperm directly into the cytoplasm of the oocyte (CDC 2012). Sixty-six percent of the 154,412 ART cycles performed in 2011 involved ICSI (SART 2013). There have been adverse outcomes in the offspring associated with the process of IVF such as increased blood pressure and peripheral body fat in children (Ceelen et al. 2007, Ceelen et al. 2008, Ceelen et al. 2009), as well as the more invasive ICSI procedure including abnormal retinal vascularization in children (Wikstrand et al. 2008).

\section{Embryo Culture}

Embryo culture is an ART procedure which allows the embryo to develop outside of the normal maternal environment. All culture media commercially used for human ART include the inorganic ions $\mathrm{Na}^{+}, \mathrm{K}^{+}, \mathrm{Cl}^{-}, \mathrm{Ca}^{2+}, \mathrm{Mg}^{2+}$ and $\mathrm{SO}_{4}{ }^{2-}$ and the presence of these ions has not changed since the original media used for mouse embryo culture in the 1950s [reviewed in (Baltz 2012)]. The original successful mouse embryo culture media was Whitten Medium (Whitten 1956). Whitten discovered that adding glucose as a carbon source and bovine serum albumin (BSA) to a physiological saline medium allowed 8-cell stage mouse embryos to develop to the blastocyst stage [reviewed in 
(Biggers 1998)]. However, it was soon realized that an ideal medium had not yet been created because many embryos arrested at the two-cell stage (Goddard 1983).

The two cell arrest is known as the two-cell block, and it coincides with the time of major zygotic genome activation in the mouse (Goddard 1983). Subsequently, adding EDTA as a chelator was found to help overcome the two-cell block (Abramczuk J 1977). EDTA acts as a chelator by binding with high affinity to $\mathrm{Cu}^{2+}$ and $\mathrm{Zn}^{2+}$ ions that can be harmful in too high of concentrations [reviewed in (Baltz 2012)]. Since that time, many culture media have been developed to try to more closely represent the natural oviductal fluid for the development of the embryo [reviewed in (Baltz 2012)], although no medium can perfectly mimic the maternal environment. The shortfalls of culture media when compared to oviductal and uterine fluids can have adverse effects on embryo development and subsequent development of the offspring (Rinaudo \& Schultz 2004, Pfeifer et al. 2012).

A major inconsistency between embryo culture and the maternal environment is that embryo culture is often performed in high oxygen tension while the normal environment in which an embryo develops is actually hypoxic. The normal oxygen content is $8 \%$ and $1.5 \%$ in the oviduct and uterus, respectively, whereas many culture protocols involve oxygen tension of around 20\% [i.e.the oxygen content of air; (Pfeifer et al. 2012)]. The adverse effects of high levels of oxygen will be discussed in a later section. 


\section{Embryo Transfer}

In human embryo transfer, embryos are produced in vitro and are transferred either into the woman who provided the oocyte for the ART procedure (autologous), or into another woman facing infertility (donor). Embryos are most-often transferred on day 3 after egg retrieval (CDC 2012). In 68.5\% of cycles, in 2010, women used their own oocytes for the ART procedures (CDC 2012). Women undergoing embryo transfer can also receive either fresh or frozen embryos. For fresh embryo transfer, ovarian stimulation is used to increase the number of oocytes available for the subsequent ART procedure. Therefore, in an autologous and fresh transfer, the embryos are transferred into a hormonally-stimulated environment. However, in frozen and donor embryo transfer, the embryo is transferred without ovarian stimulation (Kalra et al. 2011).

\section{c) Adverse Consequences Associated with ART}

ART procedures take place at a time when the developing embryo is very susceptible to the environment because they occur at a time when the embryonic genome is being activated (Goddard 1983). These procedures can cause an altered maternal environment that results in aberrant effects as early as the oocyte and early embryo.

One way that ART can affect the maternal environment is through altered hormone levels. Estradiol levels in women undergoing ovarian stimulation can be up to 20 times higher than normal physiological levels (Kalra et al. 2011). In humans, the amount of interleukin-6 (IL-6) released into the culture medium-a measure of the ability of the embryo to develop and implant—was lower from women who had undergone SO (Yu et al. 2012). Also, the uterine environment was shown to be affected in a mouse SO 
model when embryos were less likely to implant in superovulated recipients than in naturally ovulated recipients (Ertzeid \& Storeng 2001). Similar results were found in humans when women who had undergone ovarian hyperstimulation for an IVF cycle had lower implantation rates than those who underwent unstimulated IVF (Paulson 1990).

Another way ART can create an unfavorable maternal environment is through increased oxidative damage. ART has been associated with increased oxidative damage in mouse ovaries and oocytes, possibly through its effects on the mitochondria (Chao et al. 2005, Ge et al. 2012). The mitochondria, which are maternal in origin (Wagner 1972), are involved in the production of ROS; therefore, if the mitochondria are altered in the oocyte (the source of embryonic mitochondria) then they will not be able to support the development of the embryo (Dumollard et al. 2009). Multiple cycles of ovarian stimulation are not uncommon, as approximately $45 \%$ of the women who used ART with fresh, non-donor eggs in 2010 had undergone ART cycles before (CDC 2012). It has been shown that increased number of cycles of ovarian stimulation can induce changes in the mitochondria that increase oxidative damage in the resulting embryos (Chao et al. 2005).

ART has also been shown to induce epigenetic and gene expression changes in the oocyte and early embryo. SO alone has been shown to decrease global DNA methylation levels in mouse oocytes (Almamun 2011). DNA methylation is an epigenetic mark associated with regulation of gene expression. One study in mouse preimplantation embryos showed that even the highest quality embryo cultures used presently resulted in aberrant expression of genes involved in embryo development and oxidative stress regulation (Pfeifer et al. 2012). Another study in preimplantation mouse 
embryos showed misregulation of 114 genes after culture in Whitten's medium, and 29 genes after culture in KSOM supplemented with amino acids when compared to in vivo embryos (Rinaudo \& Schultz 2004). A study by Denomme et al. (2011) suggested that SO affects the embryo's ability to maintain gene imprints (Denomme et al. 2011). Imprinted genes are genes that are only expressed from one parental allele, and whose correct expression is required for proper growth and development of the fetus and the placenta (Fowden et al. 2006).

The use of ART and the change in environment during embryo development can result in aberrant programming of critical physiological systems and development. Along with effects on the preimplantation embryo, ART procedures can affect the subsequent fetal development of the offspring. The procedure of ovarian stimulation alone can cause an increased risk for low birth weight (Ertzeid \& Storeng 1992, Kalra et al. 2011). In addition, in single births from single pregnancies using fresh, non-donor oocytes, $11.7 \%$ of infants were born pre-term, and 8.6\% had low birth weight (CDC 2012). A difference in birth weight in humans born from IVF from different culture media has been observed, even when accounting for infertility (Dumoulin et al. 2010, Nelissen et al. 2012). ART has also been associated with adverse outcomes in utero including greater dysplasia, multiple gestations, risk of monozygotic twinning, and increased rates of congenital heart defects [(Schachter 2001, Katalinic et al. 2004, Olson et al. 2005) reviewed in (Zollner \& Dietl 2013)]. Adverse effects of ART can also be seen with the increased risk of loss-ofimprinting (LOI) disorders including Beckwith-Wiedemann Syndrome and Angelman Syndrome in humans, as well as the related Large Offspring Syndrome (LOS) in ruminants (Young 1998, Maher 2003, Horsthemke \& Ludwig 2005, Chen et al. 2013). 
Adverse metabolic outcomes have been observed in offspring conceived through ART. In human retrospective studies, ART was associated with higher peripheral body fat (Ceelen et al. 2007). They also found that study participants conceived using IVF had a higher fasting glucose level (Ceelen et al. 2008). In a similar study, girls that were conceived with the use of ICSI had increased peripheral adiposity at 14 years of age when compared to age matched controls that were spontaneously conceived (Belva et al. 2012). This could have possible implications for a relationship between ART and diabetes later in life. According to Chen et al., children conceived through ART also have higher levels of insulin and higher levels of triglycerides (Chen 2011).

\section{ART and Cardiovascular Abnormalities in Offspring}

The cardiovascular system has also been shown to be affected with the use of ART. Since the first person conceived with the use of ART [Louise Brown; (Edwards et al. 1980)] is in her mid-thirties and the onset of CVD is generally later in life, it cannot yet be determined whether people who were conceived using ART develop CVD at an increased rate. However, studies have been conducted in children and young adults to examine possible precursors to CVD, including increased blood pressure and abnormal vascularization.

One study examined retinal vascularization in a Swedish population of children conceived by ICSI (Wikstrand et al. 2008), as decreased retinal vascularization has been associated with an increased risk of death from coronary heart disease (Liew et al. 2011). Wikstrand et al. (2008) looked at the number of branching points in the vessels in the eye to represent vascularization. One observation that they made was that children conceived using ICSI had a lower median number of branching points than in the control children 
who were conceived naturally. Specifically, the boys conceived using ICSI had significantly fewer branching points than the boys conceived naturally. This effect was not observed in girls (Wikstrand et al. 2008).

Ceelen et al. have performed retrospective studies in children and young adults conceived with the use of IVF in the Netherlands. As mentioned previously, some of the adverse outcomes they observed in the children were increased peripheral body fat and higher fasting glucose levels (Ceelen et al. 2007, Ceelen et al. 2008). In relation to cardiovascular development, they also found a significant difference in systolic and diastolic blood pressure in participants conceived through in IVF as opposed to participants conceived naturally (Ceelen et al. 2007). In a later study, they compared children conceived using IVF to children that were born spontaneously to subfertile parents in order to control for infertility. They found that children conceived using IVF who experienced rapid growth during early childhood had higher blood pressure in the follow-up measurement at 8-18 years old, and that this was not observed in the controls (Ceelen et al. 2009).

Animal models have also been used to examine the effects of ART on the cardiovascular system. A study in mice showed that ART (i.e. SO, embryo culture and embryo transfer) can also affect the RAS (Watkins et al. 2007). Specifically, an increase in ACE was seen in the study. ACE is responsible for cleaving Angiotensin I into the active form, AngII (a vasoconstrictor). When the RAS is overactive (as in the case of increased ACE), hypertension can occur (Kaplan 2002). Since prolonged vasoconstriction results in higher levels of ROS and MMPs, leading to higher blood 
pressure (Martinez-Lemus et al. 2011), it is possible that ART might affect the blood pressure of offspring through the RAS and the production of ROS and MMPs.

In summary, these studies point to the cardiovascular system as a system that is susceptible to changes in the maternal environment. They show that different forms of ART including SO, embryo transfer, and IVF have been associated with adverse cardiovascular outcomes in children including increased blood pressure and abnormal vascularization.

\subsection{Maternal Obesity and Infertility and ART}

The previous sections have shown how perturbations in the maternal environment can affect the offspring, both in utero and in their future development. Maternal obesity is highly prevalent in today’s society, and has been associated with adverse effects on the oocytes and embryos (Igosheva et al. 2010) as well as with pregnancy complications (Sebire et al. 2001). As with ART and maternal undernutrition, maternal obesity can also result in adverse health in the offspring as adults (Nelson et al. 2010, West et al. 2011). While ART and maternal obesity are often studied alone as suboptimal maternal environments, they often coincide in reality as will be discussed in this section.

\section{a) Maternal Obesity and Infertility}

Obesity is associated with an increased incidence of infertility (Grodstein et al. 1994, Spandorfer et al. 2004, van der Steeg et al. 2008). It has been estimated that women who were obese at 18 years of age are nearly three times more likely to be 
infertile than women with lower BMI’s [20-21.9; (Rich-Edwards et al. 1994)]. An increased BMI correlates with decreased ability to conceive, even in women who ovulate regularly (Jensen et al. 1999, van der Steeg et al. 2008). However, women who are obese are also more likely to have decreased ovulation. Increased risk for anovulation, or the lack of release of an oocyte in a menstrual cycle, is found in women who are overweight or obese (Grodstein et al. 1994, Spandorfer et al. 2004). Ovulatory dysfunction accounted for approximately 7\% of the cases of infertility present in clinics in 2010 (CDC 2012).

Obese women also have an increased time to conception (Lake et al. 1997, Jensen et al. 1999). With the rise in obesity, more overweight and obese women are utilizing ART (Robker 2008). Obese (Radavelli-Bagatini et al. 2011) and HF diet (Wu et al. 2010) mouse models have also revealed increased anovulation. In a mouse model of diet induced obesity, obese mice were more likely to show anovulation and were more likely to be deemed infertile as determined by failure to breed for over 20 consecutive days (Bermejo-Alvarez et al. 2012). Consumption of a HF diet has also resulted in abnormal estrous cyclicity in rats (Balasubramanian et al. 2012).

\section{b) Reduced ART success with Maternal Obesity}

The adverse effects of obesity during ART procedures can first be observed during ovarian stimulation and oocyte collection. Increased amounts of gonadotropins are needed in ART cycles for obese women (Depalo et al. 2011, Valckx et al. 2012, Zander-Fox et al. 2012), and women who are obese have greater rates of IVF cancellation, as measured by a lack of response to exogenous gonadotropin stimulation (Spandorfer et al. 2004, Luke et al. 2011a). Further, less oocytes are able to be collected 
from obese or morbidly obese (BMI $\geq 40$ ) women undergoing ART (Spandorfer et al. 2004, Dokras et al. 2006, Valckx et al. 2012, Zander-Fox et al. 2012). Wittemer et al. (2000) showed that women who were overweight had fewer good quality MI and MII oocytes when undergoing IVF than women of normal weight [BMI 20-25; (Wittemer et al. 2000)]. Decreased oocyte quality could have an adverse effect on fertilization rates. For example, Shah et al. (2011) showed that women undergoing IVF alone or an IVF procedure including ICSI had less fertilized oocytes if their BMI was $\geq 35$ (Shah et al. 2011).

Embryo number and quality can also be affected in obese women undergoing ART procedures (Metwally et al. 2007, Zhang et al. 2010, Valckx et al. 2012). Luke et al. (2011) found that as the BMI of women undergoing IVF increased, the proportion of blastocyst-stage embryos were decreased (Luke et al. 2011b). Valckz et al. (2012) have also shown reduced blastocyst formation during IVF for women with increasing BMI's (Valckx et al. 2012). Other studies have revealed that the embryos that result from obese women undergoing IVF treatments are lower quality than women with a normal BMI (Metwally et al. 2007, Zhang et al. 2010).

Reduced oocyte competence and embryo development have also been demonstrated in animal models of obesity or maternal overnutrition. Fewer oocytes from mice consuming a HF diet were able to be fertilized in vitro (Wu et al. 2010). Wu et al. (2010) further showed that the cumulus oocyte complexes from obese females had increased lipid content and increased expression of stress marker genes (Wu et al. 2010). Further, embryos from overfed ewes who consumed more than twice the amount of food than control ewes had decreased morula and blastocyst rate in culture when compared to 
embryos from control ewes (Grazul-Bilska et al. 2012). In sheep, high levels of PUFAs decreased the development of fertilized oocytes to the blastocyst stage in culture (Wonnacott et al. 2010). Retarded embryo development in culture due to obesity has also been observed in mice (Minge et al. 2008, Binder et al. 2012). Wakefield et al.(2008) further demonstrated that feeding female mice excess PUFAs decreased the ability of their embryos to develop to the blastocyst stage in culture after in vivo fertilization and SO (Wakefield et al. 2008).

Decreased implantation rates have been observed when embryos were transferred into morbidly obese women (Zander-Fox et al. 2012) and women with BMI >25 (Depalo et al. 2011) when compared to women with normal body weights (BMI 19-24.9). A similar study that controlled for infertility by transferring an embryo from a healthy, fertile donor into a healthy, fertile recipient found a significant decrease in implantation rate with increasing BMI of the embryo recipients. (DeUgarte et al. 2010). However, many studies have also shown no change in implantation with obesity (Fedorcsak et al. 2001, Dokras et al. 2006). Despite this discrepancy, women undergoing IVF who are obese have displayed decreased pregnancy rates (Lake et al. 1997, Maheshwari et al. 2007, Zander-Fox et al. 2012).

\section{c) Adverse Pregnancy Outcomes with Obesity and ART}

Adverse outcomes due to obesity in ART pregnancies are increased risk for preeclampsia, gestational diabetes, pre-term deliveries and an increased need for cesarean section deliveries (O'Brien et al. 2003, Dokras et al. 2006, Bhattacharya et al. 2007, Dickey et al. 2012, Sauber-Schatz et al. 2012, Zander-Fox et al. 2012). Obesity during 
ART procedures can also have adverse effects on the pregnancy. In a large study performed with women undergoing ART in England, women with BMI's $\geq 27$ had a 33\% decreased chance of delivering a live birth (Lintsen et al. 2005). Increased risk of miscarriage has been observed in obese women undergoing ART procedures (Dokras et al. 2006, Bhattacharya et al. 2007, Maheshwari et al. 2007). There is also a greater miscarriage rate in obese women receiving donor oocytes from non-obese individuals implying reduced endometrial receptivity (Bellver et al. 2007). Wen et al. (2010) found that children conceived through IVF or ICSI were at a higher risk for congenital heart defects, and that this risk increased when the mothers had a BMI of 30 or above (Wen et al. 2010). Also, women who are obese and use IVF are more likely to deliver macrosomic offspring (Dokras et al. 2006, Zander-Fox et al. 2012). 


\section{RATIONALE FOR THESIS}

Previous research has shown that the maternal environment an individual is exposed to during in utero development can affect the subsequent disease state of the individual. Maternal obesity or the use of ART have been associated with adverse outcomes in the offspring including increased adiposity and blood pressure which are both associated with the development of CVD. Obese women have an increased risk for infertility, and the number of obese women who seek ART to conceive is increasing. Little is known about the effect that these two maternal environments have on the offspring when both are present simultaneously or what mechanism is involved in the increased blood pressure found in studies involving ART or maternal obesity.

High blood pressure (hypertension) has been associated with inward remodeling of the vasculature. Previous research in our laboratory suggests that the matrix metalloproteinases (MMPs) are involved in this inward remodeling. MMP activity can be increased by NADPH Oxidase (NOX)-derived reactive oxygen species (ROS), and decreased by the endogenous tissue inhibitors of matrix metalloproteinases (TIMPs).

We hypothesize that obesity and ART independently and synergistically adversely affect the cardiovascular health and body weight of the offspring. We further hypothesize that these two suboptimal maternal environments are associated with higher blood pressure in the offspring, and that this is mediated by the MMPs, TIMPs and NOXs. The research presented in Chapter III of this thesis tested these hypotheses in a mouse model. 


\section{CHAPTER 3}

\section{Effects of the Use of Assisted Reproduction and High Caloric Diet Consumption on Body Weight and Cardiovascular Health of Juvenile Mouse Offspring}

\subsection{Abstract}

Maternal obesity and the use of assisted reproductive technologies (ART) are two suboptimal maternal environments that can lead to offspring obesity and cardiovascular disease. We hypothesized that these environments independently and synergistically adversely affect the offspring's weight and cardiovascular performance at $\sim 7$ weeks of age. Mice were fed either 24\% fat and 17.5\% high fructose corn syrup (HF) or maintenance chow (5\% fat; LF). Dams were subdivided into no-ART and ART groups. ART embryos were cultured in Whitten's medium and transferred into pseudopregnant recipients consuming the same diet as the donor. Offspring were fed the same diet as the mother. Body weights were measured weekly and mean arterial pressure (MAP) was collected through carotid artery catheterization at sacrifice (55 \pm 0.5 days old). Expression of genes involved in cardiovascular remodeling was measured in thoracic aorta using qRT-PCR, and levels of reactive oxygen species (ROS) were measured intracellularly and extracellularly in mesenteric resistance arteries. ART resulted in increased body weight at weaning, but this effect decreased over time and diet was the predominant determinant of body weight by sacrifice. Males had greater MAP than females $(\mathrm{p}=0.002)$ and HF consumption was associated with greater MAP regardless of sex $(\mathrm{p}<0.05)$. Gene expression was affected by sex $(\mathrm{p}<0.05)$ and diet $(\mathrm{p}<0.1)$. Lastly, the use of ART resulted in offspring with increased intracellular ROS $(p=0.05)$. In 
summary, exposure to an obesogenic diet affects weight, MAP, and gene expression while ART increases oxidative stress in mesenteric resistance arteries of juvenile offspring, no synergistic effects were observed.

\subsection{Introduction}

Developmental priming and fetal programming have emerged as important hypotheses that partly explain the surge in obesity and cardiovascular disease (CVD) currently observed worldwide (WHO 2013a, WHO 2013b). These hypotheses, collectively known as developmental origins of adult disease, assert that the uterine environment has profound effects on the body composition and cardiovascular performance of the offspring in later life (Hales 1992, Lawlor et al. 2004).

Recent research has shown that maternal obesity, over-nutrition, and diabetes increase the incidence of glucose intolerance, arterial endothelial dysfunction, arterial stiffness, hypertension, and CVD in the offspring in humans and mice (Wildman et al. 2003, Samuelsson et al. 2008, Samuelsson et al. 2010, Kelsall et al. 2012, Torrens et al. 2012, Magliano et al. 2013). In mice, maternal obesity can result in progenies with increased body weight (Samuelsson et al. 2008, Franco et al. 2012, Magliano et al. 2013). Further, Torrens et al. showed that offspring exposed to maternal obesity had decreased production of the vasodilator nitric oxide (NO), a blunted vasodilatory response to acetylcholine in the femoral artery, as well as increased systolic blood pressure at 30 weeks of age (Torrens et al. 2012). Maternal high caloric intake in mice 
has also been linked to impaired vascular responses and increased blood pressure of the offspring during adulthood (Samuelsson et al. 2008).

The use of Assisted Reproductive Technologies (ART) has been associated with adverse outcomes in children (Olson et al. 2005, Kallen et al. 2010, Wen et al. 2010). Studies have shown that the use of in vitro fertilization (IVF) results in an increased risk for congenital malformations such as cardiac septal defects, neural tube defects and cleft palate (Olson et al. 2005, Reefhuis et al. 2009, Kallen et al. 2010). In addition, Wen et al. found that the risk for congenital heart defects with the use of ART was amplified when the mother was obese (Wen et al. 2010).

Retrospective studies in human (Wikstrand et al. 2008, Ceelen et al. 2009, Sakka et al. 2010) have also pointed at the method of conception as a causative factor for abnormal cardiovascular parameters in children during postnatal development. For example, it has been demonstrated that children conceived by the use of ART have increased systolic and diastolic blood pressure, increased vascular dysfunction, and decreased retinal vascular branching (Ceelen et al. 2008, Wikstrand et al. 2008, Sakka et al. 2010, Scherrer et al. 2012). Similarly, in mice, an association exists between ART and an elevated systolic blood pressure (Watkins et al. 2007). Further, increased angiotensin converting enzyme (ACE), which cleaves angiotensin I into the vasoconstrictor angiotensin II, has also been observed in mice offspring conceived via ART (Watkins et al. 2007). Lastly, the use of ART has been associated with increased adiposity in children (Ceelen et al. 2007, Belva et al. 2012). For example, children conceived by IVF and ICSI have higher levels of peripheral adiposity than age matched naturally conceived controls at 8-18 years of age (Ceelen et al. 2007, Belva et al. 2012). 
Cardiovascular disease is the number one cause of death. In 2008 CVD accounted for $30 \%$ of deaths worldwide (WHO 2013b). Obesity and hypertension are two important contributors to the high incidences of CVD mortality worldwide (WHO 2013b). The development of hypertension has been proposed to involve the matrix metalloproteinases (MMPs) and reactive oxygen species [ROS;(Martinez-Lemus \& Galinanes 2011)]. ROS can mediate the expression and activation of MMPs (Siwik et al. 2001, Martinez-Lemus et al. 2011, Zhang et al. 2013). In the vasculature, ROS are mainly produced by the enzyme NADPH oxidase [NOX; (Cai et al. 2003)]. MMPs are involved in processes such as the degradation of the extracellular matrix (ECM), the cleavage of extracellular receptors (Rodrigues et al. 2010), disruption of cellular adhesions (Yang et al. 2007) and shedding of vasoactive factors within the ECM (Hao et al. 2004). Specifically, MMP2 and MMP9 have been implicated in vascular remodeling, and MMP7 might also be involved as it appears to play a role in hypertension (Lehoux et al. 2004, Wang et al. 2009, Martinez-Lemus et al. 2011). Tissue Inhibitors of MMPs (TIMPs) are endogenous inhibitors of the MMPs, and an imbalance between TIMPs and MMPs has been implicated in pathological conditions (Gomez et al. 1997).

Maternal obesity and the use of ART often coincide because many women of reproductive age face infertility, and the number of them who attend ART clinics is rising (Robker 2008). It is clear that obesity and ART are each predisposing children to CVD. However, given the short history of the use of ART, no studies have yet addressed the threat of CVD from the combination of the two suboptimal maternal environments, despite the large number of children who have been exposed to it. In the current study we hypothesize that obesity and ART independently and synergistically adversely affect the 
cardiovascular health and body weight of the offspring. We further hypothesize that these two suboptimal developmental environments are associated with higher blood pressure in the offspring, and that this is associated with expression of the MMPs, TIMPs and NOXs in the vasculature. To test these hypotheses, two suboptimal developmental environments were used; namely, those that exist with an obesogenic environment and with the use of ART. We sought to determine what effect these two environments, either alone or in combination, would have on cardiovascular health markers in juvenile mice offspring ( $\sim$ weeks of age). The measures of cardiovascular health chosen in this study were MAP, the expression of genes implicated in poor cardiovascular health and vascular remodeling (Mmp2, Mmp9, Timp1, and Nox2), and levels of intracellular and extracellular ROS. We also measured offspring body weight weekly until sacrifice, as high body weight has been associated with adverse cardiovascular outcomes (NIH 2012).

\subsection{Materials and Methods}

\section{a) Animals}

All animal procedures were approved by the Institutional Animal Care and Use Committee of the University of Missouri under protocol number 7501. The strain of the females used for this experiment was NSA (CF1; Harlan Laboratories, Indianapolis, IN, USA) while the strain of the studs and vasectomized males was B6D2F1/J (The Jackson Laboratory, Bar Harbor, ME, USA). Females were between 6 and 10 weeks of age, and were housed under 12 hour (h) light/dark cycle between $20^{\circ} \mathrm{C}$ and $23.3^{\circ} \mathrm{C}$ in groups until 
conception when they were individually caged. Mice were given ad libitum access to water and the appropriate diet.

\section{b) Diet}

Experimental animals were fed either a "high fat, high fructose" (HF) or "low fat, no fructose” (LF) diet. The HF group were fed a diet containing 24\% fat and $17.5 \%$ high fructose corn syrup from TestDiet 58Y1 (TestDiet ; St. Louis, MO, USA) ad libitum. The calories (kcal\%) provided by the HF diet are as follows: protein $=17.6 \%$, carbohydrate $=36 \%$ and fat $=46.4 \%$. The females in the LF group were fed a diet containing 5\% fat (measured by ether extraction; Lab Diets 5001 PMI Nutritional International St. Louis, MO, USA) ad libitum. The calories (kcal\%) provided by the LF diet is a follows: protein $=28.5 \%$, carbohydrate $=58 \%$ and fat $=13.5 \%$. The LF females were given Lab Diet 5008 (6.5\% fat) during pregnancy and lactation. The calories (kcal\%) provided by the LF pregnancy diet are as follows: protein=26.8\%, carbohydrate $=56.4 \%$ and fat $=16.7 \%$. The HF diet administered prior to and during pregnancy and lactation was TestDiet $58 Y 1$ (46\% kcal fat with corn syrup). Note: the terms “maternal and offspring consumption of HF diet” and “exposure to an obesogenic diet” will be used interchangeably.

\section{c) Control (no ART) Groups}

The control females were fed the HF or LF diet at least 3 weeks prior to being paired with proven fertility B6D2F1/J males. Three weeks were chosen because this is the approximate time required for oocyte development in mice (Eppig et al. 2002). Copulation was confirmed by visual observation of a copulatory plug. Pregnant females 
were placed in a separate cage and were allowed to carry the pregnancy to term. Twelve total control females were paired with fertile males (6 HF and 6 LF), but one HF female did not become pregnant. After birth, the litters were culled to 8 pups (4 males and 4 females, when possible), to help control for the smaller litter sizes in the ART groups.

\section{d) Assisted Reproduction Groups}

Three ART procedures were used in this experiment, namely, superovulation, embryo collection and culture, and embryo transfer in order to simulate three procedures commonly used in human ART.

\section{Superovulation}

Females of 6-10 weeks of age fed either HF or LF diet for three weeks before superovulation received an intraperitoneal (IP) injection of 5 IU equine chorionic gonadotropin (eCG; Calbiochem La Jolla, CA, USA) followed by 5 IU of human chorionic gonadotropin (hCG; Sigma St. Louis, MO, USA) 45 h later. The injections were given to increase the number of oocytes ovulated by each animal. Superovulated females were then co-caged overnight with B6D2F1/J intact males.

\section{Embryo Collection and Culture}

Two-cell embryos were harvested from the oviduct approximately $46 \mathrm{~h}$ posthCG injection. Oviducts were flushed with warm bicarbonate-free minimal essential medium (Earle’s salt) supplemented with $3 \mathrm{mg} / \mathrm{ml}$ polyvinylpyrrolidone (PVP) and 25 mM Hepes (MEM+PVP [pH 7.3]; Sigma St. Louis, MO, USA). Embryos were washed from debris with three consecutive washes in MEM+PVP and once in Whitten's medium. Whitten's medium was prepared as previously published by us (Negron-Perez et al. 
2013). Whitten's medium has previously been shown to support development to term (Ecker et al. 2004, Sommovilla et al. 2005), although it is known to be a suboptimal culture medium (Rinaudo \& Schultz 2004). We purposely used this medium to determine whether the cardiovascular measures examined in the current study would be adversely affected by a suboptimal culture medium, as such findings could direct the focus of future studies in more favorable culture media. The embryos were cultured at $37^{\circ} \mathrm{C}$ in Whitten's medium in an atmosphere of $5 \% \mathrm{CO}_{2}$ in air at a density of 1 embryo per $\sim 3.5 \mu \mathrm{l}$ of medium. Embryos were cultured for 3 days (or $116 \mathrm{~h}$ post-hCG) at which time zona-enclosed blastocysts were transferred to the uteri of day 2.5 (d2.5) pseudopregnant NSA (CF1) females.

\section{Embryo Transfers}

Females designated as embryo recipients [NSA(CF1)] were fed HF or LF diets at the same time as the embryo donors prior to receiving the superovulation protocol described above. Immediately after the hCG injection, the females were co-caged overnight with vasectomized B6D2F1/J males. Females which showed a copulatory plug the following morning (denoted as $\mathrm{d} 0.5$ of pseudopregnancy) were selected as potential embryo recipients. A plane of anesthesia was reached by an IP injection of the $2.5 \%$ (w/v) Avertin stock at $0.014 \mathrm{ml} / \mathrm{g}$ body weight (Nagy A 2003). Twenty blastocyst-stage embryos produced as described above were transferred (ten/uterine horn) to embryo recipients on d2.5 of pseudo-pregnancy according to standard procedures (Nagy A 2003). Pseudopregnant recipients were consuming the same diet as the embryo donor. Pregnant recipients were returned to their cages and allowed to carry their pregnancies to term. 
Forty-five transfers were performed (22 LF and $23 \mathrm{HF}$ ). Nearly 27\% of transfers resulted in pregnancy (determined by birth of offspring; Supplementary Table 1).

\section{e) Pregnancy, Lactation and Weaning}

All females were maintained on the diet they had been consuming prior to becoming pregnant until their role in the experiment was completed at weaning. Offspring were weaned from the mothers at 22 days post-partum. They were separated by sex and placed on the same diet that the mothers had consumed until sacrifice.

\section{f) Measurement of Offspring Body Weights}

Body weight was recorded using a standard scale (Mettler Toledo; Columbus, OH, USA) five days after birth, and every week thereafter until sacrifice. Control litters were culled to eight (4 males and 4 females when possible) at the 5-day weighing to control for the larger litter sizes in the control groups. No identification marks were used prior to weaning; therefore, weights were recorded separately for males and females from each litter and averaged each week during the first three weeks. Each individual received an identifying ear punch at weaning, and weights were recorded by individual from that point on.

\section{g) Mean Arterial Pressure Measurement}

Offspring male $(\mathrm{n}=37)$ and female $(\mathrm{n}=31)$ mice $55.5 \pm 0.5(\mathrm{n}=68)$ days old were anesthetized by inhalation of $5 \%$ isoflurane until visible loss of consciousness. At that point, mice were placed in dorsal recumbency and maintained under anesthesia at 3\% $(\mathrm{v} / \mathrm{v})$ through a cone covering the nose and mouth until surgical plane anesthesia was confirmed by loss of spinal reflexes. The right carotid artery was cannulated with a 
polyethylene catheter (PE-10) filled with phosphate-buffered physiological saline solution containing $100 \mathrm{U} / \mathrm{mL}$ of heparin. The catheter was connected to a pressure transducer in order to measure mean arterial pressure (MAP) with the use of a PowerLab 4/30 Data Acquisition System (ADInstruments Colorado Springs, CO,USA). Following catheterization, the level of anesthesia was lowered to $2 \%(\mathrm{v} / \mathrm{v})$ for $10 \mathrm{~min}$., and the average systolic and diastolic pressures recorded during the final minute were used to calculate MAP. It should be noted that three animals had bleeding during catheterization, and were excluded from the MAP analysis as blood loss can affect pressure.

\section{h) Mesenteric Resistance Artery and Thoracic Aorta Collection}

Subsequent to MAP measurement, animals were euthanized under anesthesia. A portion of the mesentery was excised and pinned flat in a refrigerated $\left(4^{\circ} \mathrm{C}\right)$ dissecting chamber containing physiological saline solution (PSS) of the following composition (in mmol/L): $145.0 \mathrm{NaCl}, 4.7 \mathrm{KCl}, 2.0 \mathrm{CaCl}_{2}, 1.2 \mathrm{MgSO}_{4}, 1.0 \mathrm{NaH}_{2} \mathrm{PO}_{4}, 5.0$ dextrose, 3.0 3(N-morpholino) propanesulfonic acid (MOPS) buffer, 2.0 pyruvate, 0.02 EDTA, and 0.15 bovine serum albumin (BSA), pH 7.4. Small pieces ( 3mm length) of second order mesenteric resistance arteries were isolated and stored at $-70^{\circ} \mathrm{C}$ for subsequent analysis of ROS using high performance liquid chromatography (HPLC). A small section ( 5mm length) of the thoracic aorta was also collected and stored at $-70^{\circ} \mathrm{C}$ for quantitative RTPCR analysis. Due to time constraints for the processes performed, tissues were only collected for two animals per day. In order to keep the age average similar between the ART and No ART groups, 34 of the 36 ART offspring and 34 of the 87 No ART controls were collected and used for the remainder of the study. The collected offspring were selected to have at least one male and one female for each mother. Two or three offspring 
were collected from all of the control females except for the last LF control female (three males and three females were collected) and the last two HF control females (two males and two females were collected). All ART-conceived pups were collected except for two HF males who had advanced too far in age by the time of collection.

\section{i) Dihydroethidium Incubation of Single Mesenteric Resistance Arteries for Detection of ROS}

Isolated pieces of mesenteric resistance arteries were taken from the $-70^{\circ} \mathrm{C}$ freezer, placed in $99 \mu \mathrm{L}$ 1x phosphate buffered saline (PBS; $137 \mathrm{mM} \mathrm{NaCl}, 2.7 \mathrm{mM} \mathrm{KCl}$, $10 \mathrm{mM} \mathrm{Na}_{2} \mathrm{HPO}_{4}, 1.8 \mathrm{mM} \mathrm{KH} \mathrm{PO}_{4}$ ) with $100 \mu \mathrm{M}$ DTPA (Sigma-Aldrich St. Louis, MO, USA) and incubated in a $37^{\circ} \mathrm{C}$ water bath for $10 \mathrm{~min}$. The $100 \mu \mathrm{M}$ DTPA was prepared as previously described (Buettner 2008). Briefly, $0.1967 \mathrm{~g}$ DTPA was added to $1.7 \mathrm{~mL}$ $1 \mathrm{M} \mathrm{NaOH}$ to make the DTPA go into solution more easily. The flask was then filled to approximately $40 \mathrm{~mL}$ with ultrapure $\mathrm{H}_{2} \mathrm{O}$. The solution was sonicated to bring the DTPA into solution. Then, the solution was adjusted to a $\mathrm{pH}$ of 7.1 using a $1 \mathrm{M} \mathrm{HCl}$ solution before bringing the solution to a final volume of $50 \mathrm{~mL}$ with ultrapure $\mathrm{H}_{2} \mathrm{O}$. A $5 \mathrm{mM}$ stock solution of dihydroethidium (DHE) was made by dissolving $1 \mathrm{mg}$ DHE (Molecular Probes-Life Technologies Grand Island, NY, USA) in $634 \mu \mathrm{L}$ DMSO (Sigma St. Louis, MO, USA). The resulting solution was separated into $15 \mu \mathrm{L}$ aliquots and lyophilized for $2 \mathrm{~h}$. The lyophilized DHE aliquots were stored at $-20^{\circ} \mathrm{C}$ until use. On each day of the experiment, the lyophilized DHE was resuspended in $15 \mu \mathrm{L}$ pure HPLC grade

acetonitrile. After the initial incubation, $1 \mu \mathrm{L}$ of a $5 \mathrm{mM}$ DHE stock in acetonitrile was added to the solution bathing the vessel to bring the final concentration to $50 \mu \mathrm{M}$ DHE. Ambient light was avoided to diminish excess oxidation of the DHE. The resistance 
artery was incubated with DHE at $37^{\circ} \mathrm{C}$ in the water bath for $1 \mathrm{~h}$, with gentle agitation every $10 \mathrm{~min}$. The vessel was then removed and washed twice, consecutively, in $400 \mu \mathrm{L}$ 1xPBS.

\section{j) Fluorescence Confocal Microscopy for Detection of Intracellular ROS}

After being washed in 1xPBS, the vessels were placed on a glass slide in a drop of 1xPBS and covered with a 1.5 thickness coverslip. Two random areas were chosen to be imaged for each vessel. Images were obtained using a Leica True Confocal ScanningSpectral Photometric 5 DMI 6000 Confocal Laser Scanning Microscope (Leica TCS SP5). A 3D image was obtained for each section at a photomultiplier gain of $800 \mathrm{~V}$ using a 20x dry lens. DHE was detected using a multi-photon excitation of $800 \mathrm{~nm}$ and an emission detection in the range of 600-700 nm. DHE has previously been used to detect superoxide in arterioles (Martinez-Lemus et al. 2011), as it forms a red fluorescent product upon oxidation (Owusu-Ansah et al. 2008). Laser scanning was performed using a resonator set at $8000 \mathrm{~Hz}$ to reduce photo bleaching. Z-sections were set at 0.5 $\mu \mathrm{M}$ to cover the entire vessel. All images were taken at the same excitation power and detection perameters. Analysis of relative DHE fluorescence was performed using Imaris 7.6.1 software over an area of 150x150x20 pixels for each vessel. Values for DHE fluorescence were averaged over the two areas of the vessel that were imaged. Readings for intracellular ROS were excluded if there was discrepancy in the relative DHE fluorescence between the two areas on the vessel from the same individual. 


\section{k) High Performance Liquid Chromatography (HPLC)}

HPLC was performed using $50 \mu \mathrm{L}$ of the original DTPA+1xPBS+DHE solution used to bathe the resistance artery in order to detect the oxidation of DHE outside the cells, which is a measure of extracellular ROS production. A C8 reverse phase column (Phenomenex Torrence, CA, USA) was used to separate the two byproducts of DHE oxidation, 2-hydroxyethidium (2-OH-Et) and ethidium (Et). The phase solutions used for product separation were Solution A (ultrapure water), Solution B (99.9\% v/v water with $0.1 \% \mathrm{v} / \mathrm{v}$ TFA), and Solution C (99.9\% v/v acetonitrile with 0.1\% v/v TFA; Fischer Pittsburgh, PA, USA). The samples were placed in a Waters 2590 Separation Module and 2-OH-Et and Et fluorescence were detected using a Waters 474 Scanning Fluorescence detector set at an excitation of $510 \mathrm{~nm}$ and emission of $595 \mathrm{~nm}$. Each day before running samples, the column was cleaned by running $99.9 \% \mathrm{v} / \mathrm{v}$ acetonitrile with $0.1 \% \mathrm{v} / \mathrm{v}$ TFA for $20 \mathrm{~min}$. at a flow of $1 \mathrm{ml} / \mathrm{min}$., followed by $100 \%$ ultrapure water at a flow of $0.5 \mathrm{ml} / \mathrm{min}$. for $20 \mathrm{~min}$. This cleaning was repeated after every 4 or 5 samples to stop the pressure in the column from increasing. For each sample being run, a gradient of acetonitrile $(\mathrm{v} / \mathrm{v})$ in water was used to elute the samples. The gradient was run at a flow of $0.4 \mathrm{ml} / \mathrm{min}$. with an increase in acetonitrile from $10 \%$ to $46 \%$ in the first $10 \mathrm{~min}$., an increase again to $100 \%$ from min. 20 to 25 , and ending with the original $10 \%$ acetonitrile from 25 to 35 min. to clean the column. The 2-OH-Et eluted at $16.19 \pm 0.03$ min. and the Et eluted at $16.61 \pm 0.03 \mathrm{~min}$. (See Fig. 3.4A for a representative example). The Empower program (Waters, Build \#1154) was used to extract the areas under the 2-OHEt and Et peaks detected by the fluorescence detector. One vessel for a LF No ART 
control male was lost during transfer to the DHE incubation step, so this observation is not included.

\section{l) Homogenizing Vessels for Protein Measurement}

An Omni Bead Ruptor 24 Homogenizer (Omni International Kennesaw, GA, USA) was used to pulverize the resistance arteries. After the confocal image was taken, the vessels were placed in a $0.5 \mathrm{~mL}$ tube with a cap (Omni International Kennesaw, GA, USA) with $100 \mu \mathrm{L}$ pure HPLC grade acetonitrile and $50 \mathrm{mg}$ of $0.1 \mathrm{~mm}$ glass beads (Omni International Kennesaw, GA, USA). The program for homogenization was performed at $22^{\circ} \mathrm{C}$. There were 3 cycles, each at a speed of $8 \mathrm{~m} / \mathrm{s}$ for $45 \mathrm{sec}$., with a 3 sec. wait time between cycles. The tubes were centrifuged briefly after homogenization to ensure the beads were at the bottom of the tube, and $60 \mu \mathrm{L}$ of the supernatant was recovered. This supernatant was sonicated for $1 \mathrm{~min}$. to further lyse the tissue. Then, the solution was centrifuged at $2000 x g$ for 10 min., and $50 \mu \mathrm{L}$ of the supernatant was removed. The remaining $10 \mu \mathrm{L}$ was used for protein measurement using a Micro BCA Kit.

\section{m) Protein Assays using MicroBCA Kit}

Each sample was diluted 1:4 (v/v) in pure HPLC grade acetonitrile (Fischer Pittsburgh, PA, USA) prior to protein detection. The Micro BCA ${ }^{\mathrm{TM}}$ Protein Assay Kit (Pierce Rockford, IL, USA) was used to detect the concentration of protein for each sample. A standard curve with bovine serum albumin (BSA) was generated using a blank (pure acetonitrile) and the concentrations from $1 \mu \mathrm{g}$ to $200 \mu \mathrm{g} / \mathrm{mL}$ in acetonitrile. The working reagent was prepared in accordance with manufacturer's instructions. 
Reactions were prepared by mixing $4 \mu \mathrm{L}$ of the BSA standard or diluted sample with 4 $\mu \mathrm{L}$ of the working reagent. The reactions were incubated at $60^{\circ} \mathrm{C}$ for $1 \mathrm{~h}$, and then allowed to cool to room temperature. A Nanodrop spectrophotometer was used to measure the absorbance of each sample at $562 \mathrm{~nm}$. A standard BSA curve was performed each day, and the sample absorbances were plotted against the standard curve to determine the concentration in $\mu \mathrm{g} / \mathrm{mL}$.

\section{n) RNA Extraction from Thoracic Aorta and RT-PCR}

RNA isolation was performed on thoracic aorta segments using the DynaBEADS mRNA DIRECT KIT (Invitrogen, Grand Island, NY, USA). The DynabeadOligo (dT)25 beads were equilibrated according to the manufacturer's instructions. For RNA and DNA isolation, thoracic aorta segments were placed in $200 \mu \mathrm{L}$ lysis buffer $(100 \mathrm{mM}$ Tris-HCl, 500 mM LiCl, 10 mM EDTA, 1\% LiDS, 5 mM dithiothreitol), pulverized with a DNase-RNase free pestel and then sheared by passing through a 20 gauge needle followed by a 22 gauge needle. Isolation of mRNA separated from the beads was then performed following manufacturer's specifications. The final wash of the beads was performed in $20 \mu \mathrm{L}$ sterile water, and then heated at $80^{\circ} \mathrm{C}$ for $2 \mathrm{~min}$. to separate the mRNA from the beads. The water with the mRNA was quickly removed from the beads, and $3 \mu \mathrm{L}$ was used for the creation of a $60 \mu \mathrm{L}$ cDNA reaction using $100 \mathrm{U}$ Superscript II Reverse Transcriptase (Invitrogen, Grand Island, NY, USA) and the following (1X FirstStrand Buffer, $10 \mathrm{mM}$ DTT, $0.8 \mathrm{mM}$ dNTPs, $0.5 \mu \mathrm{g}$ random primers, $44 \mathrm{U}$ RNasin). The reaction was incubated at $42^{\circ} \mathrm{C}$ for $1 \mathrm{~h}$ followed by $95^{\circ} \mathrm{C}$ for $10 \mathrm{~min}$. One microliter of the resulting cDNA was used for each qRT-PCR reaction. Procedures were followed using manufacturer's instructions, thus a template quantification step was not used. 


\section{o) Quantitative Real-Time PCR for Gene Expression in Thoracic Aorta}

The TaqMan Gene Expression Assays (Applied Biosystems Life Technologies Grand Island, NY, USA) used are shown (Table 3.1). No DNase treatment was performed as cDNA preparation was performed per the manufacturer's instructions. However, as an extra precaution and to ensure no DNA amplification, all TaqMan probes used were intron-spanning. After reverse transcription, cDNA $(1 \mu \mathrm{L})$ underwent realtime PCR amplification with $10 \mu \mathrm{L} 2 \mathrm{X}$ TaqProbe qPCR Mastermix-low ROX (BEQPCR-PL; MidSci St. Louis, MO, USA), $8 \mu \mathrm{L}$ sterile water, $1 \mu \mathrm{L}$ 20X TaqMan Gene Expression Assays probe [(Life Technologies Grand Island, NY, USA); see Table 3.1 for probe information]. The reactions were performed in triplicate using a 7500 Real Time PCR Machine (Applied Biosystems Grand Island, NY, USA) with the following cycles: $50^{\circ} \mathrm{C}-2 \mathrm{~min} . ; 95^{\circ} \mathrm{C}-1 \mathrm{~min}$; 40 cycles of $95^{\circ} \mathrm{C}-15$ sec.; $60^{\circ} \mathrm{C} 1 \mathrm{~min}$. Genes analyzed were Mmp2 (Mm00439498_m1), Mmp7 (Mm00487724_m1), Mmp9 (Mm00442991_m1), Timp1 (Mm00441818_m1) and Cybb (Nox2; Mm01287743_m1). The average threshold cycles $\left(\mathrm{C}_{\mathrm{T}}\right)$ for the cDNA were normalized to the expression level of beta-2 microglobulin (B2m; Mm00437762_m1). B2m is a gene involved in immune response as a member of the major histocompatability complex class 1 , and is not involved in energy metabolism (Shertzer et al. 2013). B2m has been previously shown to be stable in high-fat diet induced oxidative stress in adipose tissue (Bailey-Downs et al. 2013), and in response to oxidative stress in the brain (Shertzer et al. 2013) and lung (Shimada et al. 2009). A $\mathrm{C}_{\mathrm{T}}$ above 35 was considered to be not expressed, and measurements with standard deviations above 0.5 were discarded and repeated. Two LF male ART offspring were unable to be analyzed for gene expression due to complications 
during mRNA isolation. Five or six No ART controls were selected randomly from each group (HF males, LF males, HF females, LF females) to be used for gene expression analysis.

\section{p) Statistical Analysis}

The logarithms of offspring body weights were regressed on the ART effect (pregnancy through ART or not), diet effect (HF or LF), sex effect (female or male), time effect (weaning, $4^{\text {th }}$ week, $5^{\text {th }}$ week, $6^{\text {th }}$ week, and $7^{\text {th }}$ week since birth), as well as interaction effects including sex by diet, ART by time, and diet by time. Mothers are random effects in the model to capture the correlations between offspring that have the same mother. Since repeated measurements for the same offspring were taken at all time points, compound symmetry correlation structure was used to model the correlations among these observations. The model was constructed and chosen by biological considerations and diagnostic statistics. The main effects and interaction effects were all statistically significant at level 0.05 . The compound symmetry correlation structure for modeling the repeated measurements on the same offspring was chosen for its highest likelihood and lowest Akaike information criterion (AIC), Bayesian information criterion (BIC) statistics. The studentized residual plot and normal quantile-quantile (QQ) plot suggest a consistent normality assumption, and do not indicate any obvious outliers.

At the time of sacrifice, correlations were computed and tested between the logarithms of body weights, intracellular ROS, extracellular ROS (2-OH-Et and Et), and the differences in cycle thresholds of Mmp2, Mmp9, Timp1 and Nox2. 


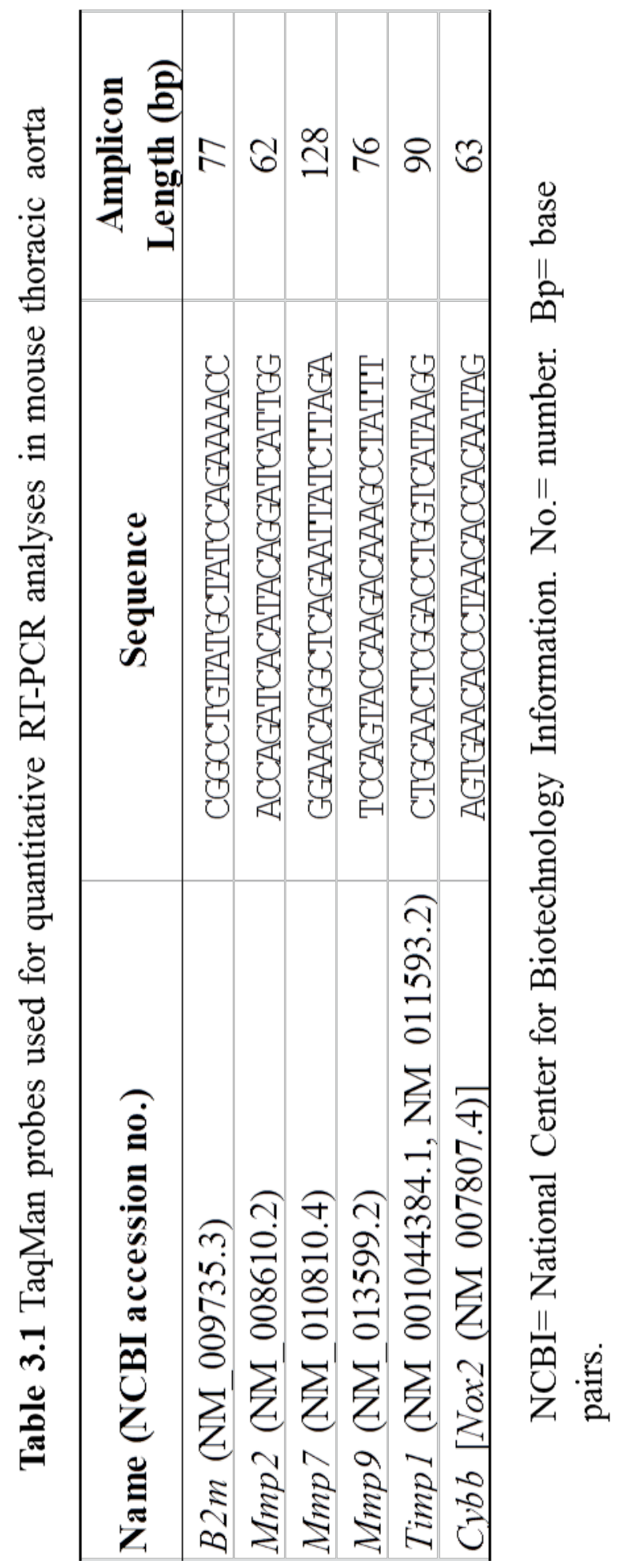


We also examined the associations between the diet, ART, and sex effects with MAP and gene expression. The significance of each of the three effects (diet, ART and sex) was tested within fixed levels of the other two. For example, the association between diet and MAP (or gene expression) was tested within fixed levels of ART and sex.

\subsection{Results}

\section{a) Effects of ART and Diet on Body Weight from Weaning to Sacrifice} Increased offspring body weight has been observed separately in models of maternal obesity and ART (Ceelen et al. 2007, Samuelsson et al. 2008, Nelson et al. 2010, Belva et al. 2012, Morandi et al. 2012). We sought to determine whether the combination of ART and high fat diet consumption by the mothers and offspring would amplify the increase in body weight expected in the offspring. The use of ART resulted in offspring with increased body weight when compared to no ART controls (No ART; p<0.0001) at weaning (i.e. 22 days of age; Fig. 3.1A). However, this effect diminished over time as the No ART group began to catch up in growth and resulted in nonsignificant body weight differences at sacrifice (i.e. $55 \pm 0.5$ days; $\mathrm{p}=0.1052$ ).

Additionally, maternal and offspring consumption of a HF diet resulted in increased body weight of the offspring from weaning ( $<<0.0001$ ) until sacrifice (Fig. 3.1B; $\mathrm{p}<0.0001$ ). Male offspring weighed significantly more than females from weaning until sacrifice $(\mathrm{p}<0.0001$; Fig. 3.1C). No effect of the interaction between diet and ART was observed in this study ( $>>0.05$; data not shown). However, there was an interaction between diet and sex. Males in the HF group had a greater increase in body weight when compared 

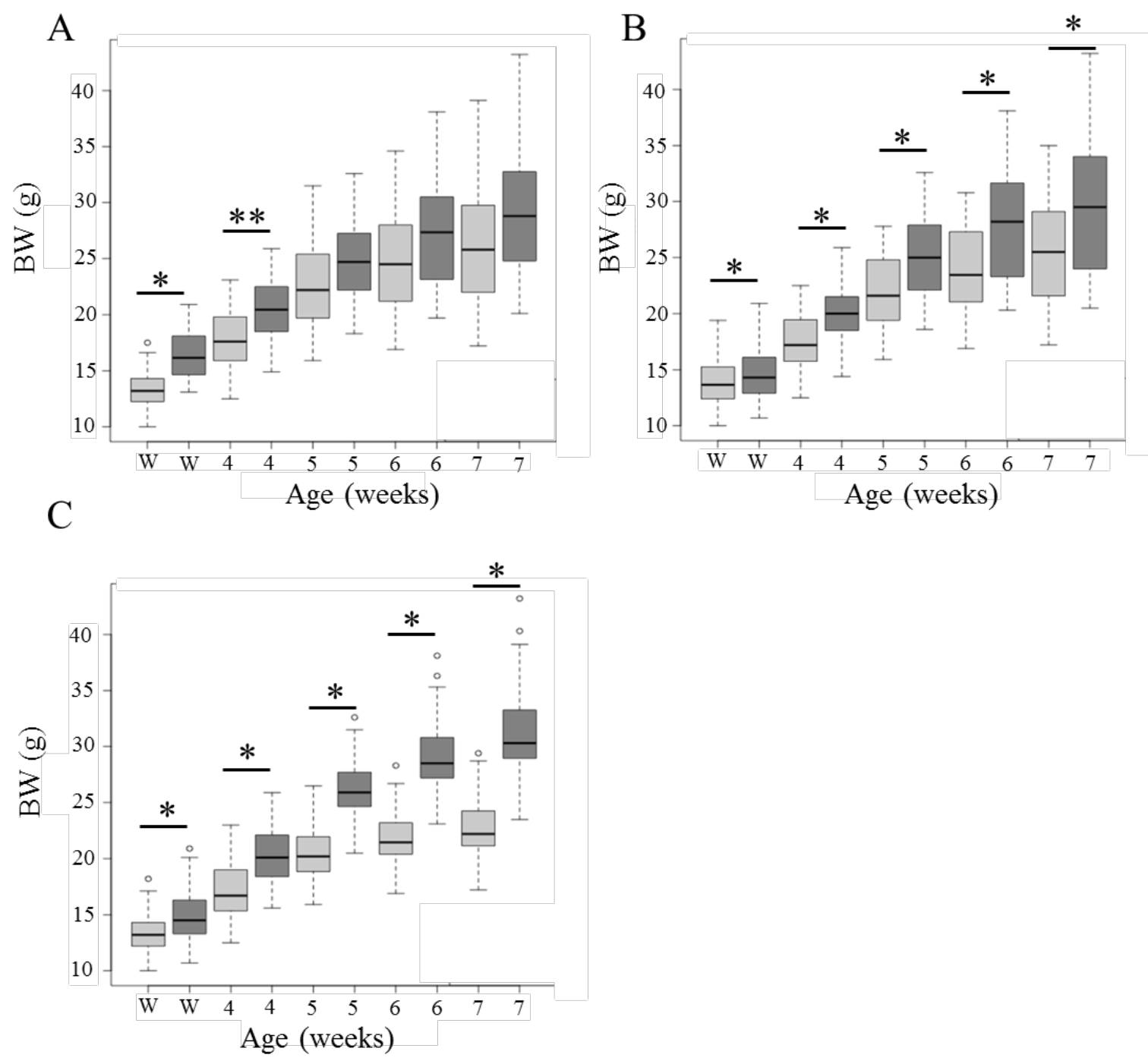

Figure 3.1. Effects of ART, diet and sex on mouse offspring body weight

from weaning until seven weeks of age. A. Body weights for all offspring conceived by natural conception (No ART; $n=87$; light grey bars) or with the use of ART (ART; $n=36$;

dark grey bars). B. Body weights for all offspring conceived by from mothers consuming a maintenance chow (LF; $n=68$; light grey bars) or a diet containing $24 \%$ fat and $17.5 \%$ high fructose corn syrup (HF; $n=55$; dark grey bars). C. Body weights of females ( $n=60$; light grey bars) and males ( $\mathrm{n}=63$; dark grey bars). Comparisons were made at weaning (22 days), week 4 (26 days), week 5 (33 days), week 6 (40 days) and week 7 (47 days). The middle bar in a box shows the median (50\%) body weight. The upper and lower borders of a box show the $1^{\text {st }}$ and $3^{\text {rd }}$ quartiles (25\% and $75 \%$, respectively) of a group body weight. The bars above or below the dash lines mark the body weights that are 2.5 times of a quartile away from the median. Dots outside of bars are even further away from the median. ART = assisted reproductive technologies. BW = body weight. Solid black lines with asterisks above are used to demarcate statistical differences between groups at given times after birth. ${ }^{*}=\mathrm{p}<0.0001,{ }^{*} *=\mathrm{p}<0.05$. 
to the LF males at sacrifice than the HF females did when compared to the LF females $(\mathrm{p}<0.03 ; 34.03 \pm 0.71 \mathrm{~g}$ vs. $28.77 \pm 0.41 \mathrm{~g}$ and $24.12 \pm 0.48 \mathrm{~g}$ vs. $21.91 \pm 0.37 \mathrm{~g}$, respectively).

\section{b) Effects of Sex and Diet on Mean Arterial Pressure}

Hypertension is another common risk factor for the development of CVD (WHO 2013b). We used mean arterial pressure (MAP; $1 / 3$ systolic $+2 / 3$ diastolic pressure) as a measure of blood pressure in the offspring. Overall, males had a higher MAP than females at sacrifice ( $p=0.002$; Fig. 3.2A). This increased MAP was associated with an increased body weight at seven weeks of age in males when compared to females $(\mathrm{p}<0.0001$; Fig. 3.1C). Further, MAP was positively correlated with body weight at sacrifice $(\mathrm{p}<0.02$; Table 3.2). Diet also had an effect on offspring MAP $(\mathrm{p}<0.05)$ after taking into account ART and sex effects. When offspring consumed a HF diet after developing in a HF maternal environment, they had higher MAP than offspring that had been exposed to a LF diet throughout (Fig. 3.2B). We did not observe increased blood pressure due to ART ( $>0.05$, Supplemental Fig. 3.1).

\section{c) Gene Expression in Response to Diet and Sex}

Altered activities of MMP2, MMP7, MMP9, TIMP1 and NOX-derived ROS have been associated with CVD (Gomez et al. 1997, Lehoux et al. 2004, Wang et al. 2009). Further, MMP2 and 9 have been suggested to be involved in ROS-dependent vascular remodeling associated with hypertension (Martinez-Lemus et al. 2011). We examined gene expressions of Mmp2, Mmp7, Mmp9, Timp1 and Nox2 in the thoracic aorta of offspring from the different treatment groups to determine if these possible markers of 
A

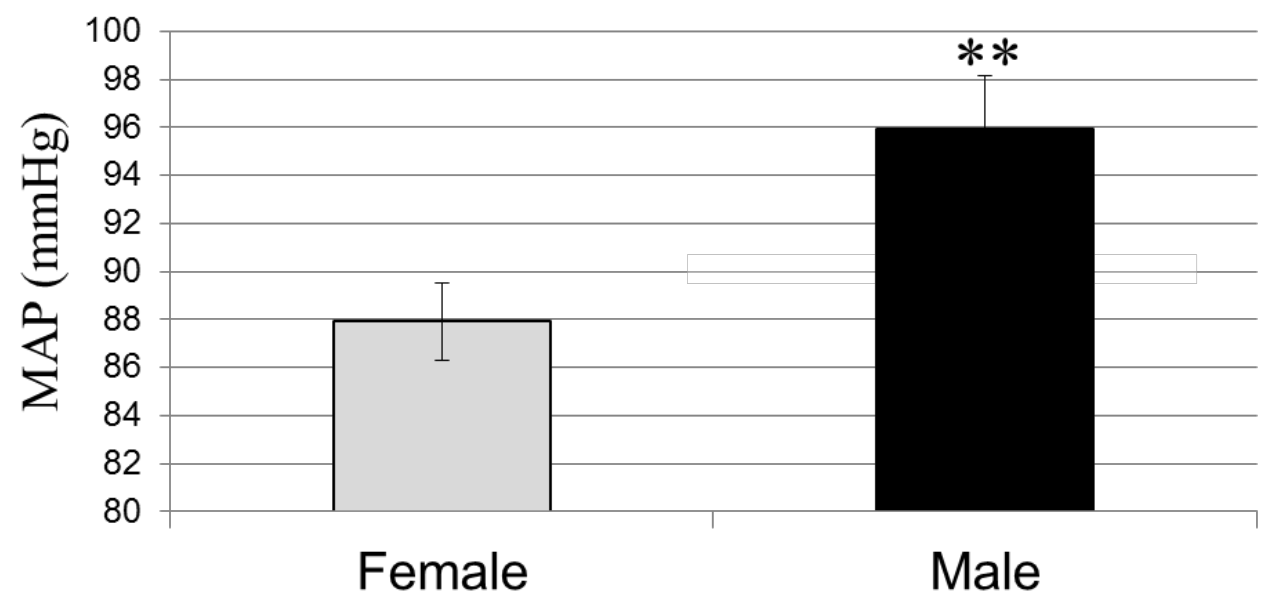

B



Figure 3.2. Mean arterial pressure (MAP) in offspring at sacrifice. Carotid artery catheterization was performed to measure MAP as a representation of blood pressure in the offspring immediately prior to sacrifice ( $55 \pm 0.5$ days). A. MAP in female $(\mathrm{n}=29)$ and males ( $\mathrm{n}=33)$, offspring. B. MAP in $\mathrm{LF}(\mathrm{n}=37)$ and $\mathrm{HF}(\mathrm{n}=25)$ offspring. The bars represent the mean \pm the S.E.M. LF=maternal and offspring consumption of a maintenance chow. $\mathrm{HF}=$ maternal and offspring consumption of a diet containing $24 \%$ fat and $17.5 \%$ high fructose corn syrup. ${ }^{*} \mathrm{p}<0.05 .{ }^{* *} \mathrm{p}<0.01$. 
Table 3.2 Table of significant correlations between measurements in mouse offspring.

\begin{tabular}{|c|c|c|c|c|c|c|c|c|}
\hline & Mmp2 & Mmp9 & Timp1 & Nox 2 & 2-OH-Et & Et & SacBW & MAP \\
\hline \multirow{3}{*}{ Mmp2 } & & 0.71722 & 0.74208 & 0.37357 & -0.36414 & -0.27963 & 0.31696 & 0.22528 \\
\hline & & $<0.0001$ & $<0.0001$ & 0.0059 & 0.0080 & 0.0447 & 0.0208 & 0.1120 \\
\hline & & 53 & 53 & 53 & 52 & 52 & 53 & 51 \\
\hline \multirow{3}{*}{ Mmp9 } & 0.717 & & 0.63446 & 0.54996 & -0.38716 & -0.36276 & 0.25921 & 0.11085 \\
\hline & $<0.0001$ & & $<0.0001$ & $<0.0001$ & 0.0046 & 0.0082 & 0.0609 & 0.4387 \\
\hline & 53 & & 53 & 53 & 52 & 52 & 53 & 51 \\
\hline \multirow{3}{*}{ Timp1 } & 0.74208 & 0.63446 & & 0.47868 & -0.42027 & -0.29326 & 0.17788 & 0.15661 \\
\hline & $<0.0001$ & $<0.0001$ & & 0.0003 & 0.0019 & 0.0349 & 0.2026 & 0.2742 \\
\hline & 53 & 53 & & 53 & 52 & 52 & 53 & 51 \\
\hline \multirow{3}{*}{ Nox 2} & 0.373357 & 0.54996 & 0.47868 & & -0.33790 & -0.25759 & -0.00719 & 0.22264 \\
\hline & 0.0059 & $<0.0001$ & 0.0003 & & 0.0143 & 0.0652 & 0.9539 & 0.1163 \\
\hline & 53 & 53 & 53 & & 52 & 52 & 53 & 51 \\
\hline \multirow{3}{*}{ 2-OH-Et } & -0.36414 & -0.38716 & -0.42027 & -0.33790 & & 0.84805 & 0.00958 & -0.26383 \\
\hline & 0.0080 & 0.0046 & 0.0019 & 0.0143 & & $<0.0001$ & 0.9387 & 0.0399 \\
\hline & 52 & 52 & 52 & 52 & & 67 & 67 & 61 \\
\hline \multirow{3}{*}{ Et } & -0.27963 & -0.36276 & -0.29326 & -0.25759 & 0.84805 & & 0.09509 & -0.13643 \\
\hline & 0.0447 & 0.0082 & 0.0349 & 0.0652 & $<0.0001$ & & 0.4440 & 0.2944 \\
\hline & 52 & 52 & 52 & 52 & 67 & & 67 & 61 \\
\hline \multirow{3}{*}{ SacBW } & 0.31696 & 0.25921 & 0.17788 & -0.00719 & 0.00958 & 0.09509 & & 0.29531 \\
\hline & 0.0208 & 0.0609 & 0.2026 & 0.9539 & 0.9387 & 0.4440 & & 0.0198 \\
\hline & 53 & 53 & 53 & 53 & 67 & 67 & & 62 \\
\hline \multirow{3}{*}{ MAP } & 0.22528 & 0.11085 & 0.15661 & 0.22264 & -0.26383 & -0.13643 & 0.29531 & \\
\hline & 0.1120 & 0.4387 & 0.2724 & 0.1163 & 0.0399 & 0.2944 & 0.0198 & \\
\hline & 51 & 51 & 51 & 51 & 61 & 61 & 62 & \\
\hline
\end{tabular}

The logarithms of the $\Delta \mathrm{CT}$ for matrix metalloproteinase 2 and 9 (Mmp2 and 9), tissue inhibitor of matrix metalloproteinase 1 (Timp1), and NADPH Oxidase 2 (Nox2) expression in thoracic aorta, extracellular 2-hydroxyethidium (2-OH-Et) and ethidium (ET) in mesenteric arterioles, body weight at sacrifice (SacBW) and mean arterial pressure (MAP) were compared using correlational analysis. The values in each cell from top to bottom represent the Pearson correlation coefficient, $\mathrm{p}$-value and number of measurements; respectively. Boldface $=$ significance at $\mathrm{p}<0.05 . \Delta \mathrm{CT}=$ difference in cycle threshold. 
cardiovascular health were affected by the two treatments alone or in combination. Offspring who were exposed to an obesogenic diet had lower expression of Mmp9 and a tendency towards lower expression of Mmp2 when compared to offspring in the LF group ( $<<0.05$ and $p<0.1$, respectively; Fig. 3.3A and C). Further, males had decreased expression of Mmp2 and the inhibitor Timp1 ( $<<0.05$ and $\mathrm{p}<0.04$, respectively; Fig. 3. 3B and D) when compared to females. No difference in expression of Nox2 was observed between treatment groups ( $\mathrm{p}>0.05$, data not shown). Also, the use of ART did not show a significant difference in the expression of any of the genes assayed in this study ( $>$ >0.05, Supplemental Fig. 3.2). Mmp7 expression was not detected in the thoracic aorta of the offspring from any treatment.

Several correlations with gene expression were observed in the offspring. The expression of all of the genes analyzed (Mmp2, Mmp9, Timp1 and Nox2) were positively correlated with each other $(\mathrm{p}<0.01$; Table 3.2). The differences in cycle thresholds of Mmp2 also had a positive correlation and Mmp9 tended to have a positive correlation with body weight at sacrifice ( $p=0.02$ and $p=0.06$, respectively; Table 3.2 ). Therefore, as the body weight increased, the expression of $M m p 2$ decreased and $M m p 9$ tended to decrease.

\section{d) Effect of ART on ROS in Offspring Mesenteric Resistance Arteries}

Previous research has shown increased oxidative stress, which can occur due to increased ROS, in obesity as well as with the use of ART (Chao et al. 2005, Torrens et al. 2012). We examined the levels of intracellular or extracellular ROS in mesenteric resistance arteries of the experimental offspring. Offspring produced by ART had 


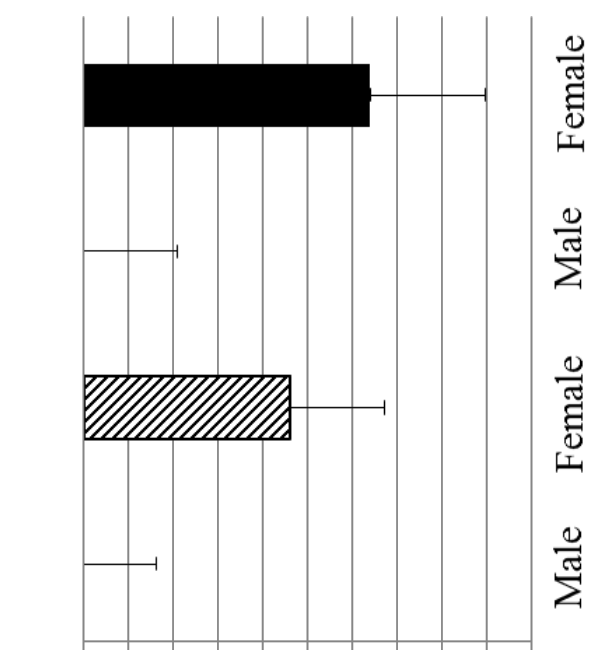

○ әре 07 әм!ре|әу

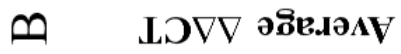

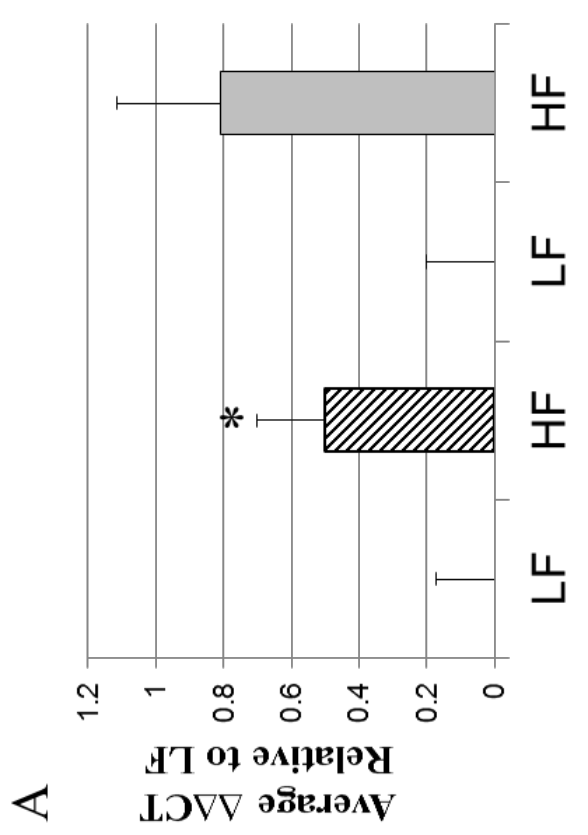

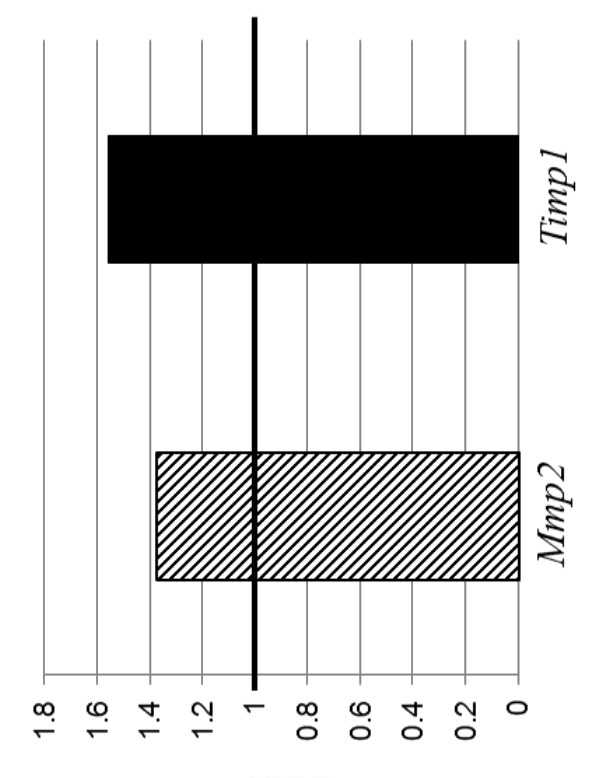

uzg

๑ шо.ј әәчә.әу!С рІо

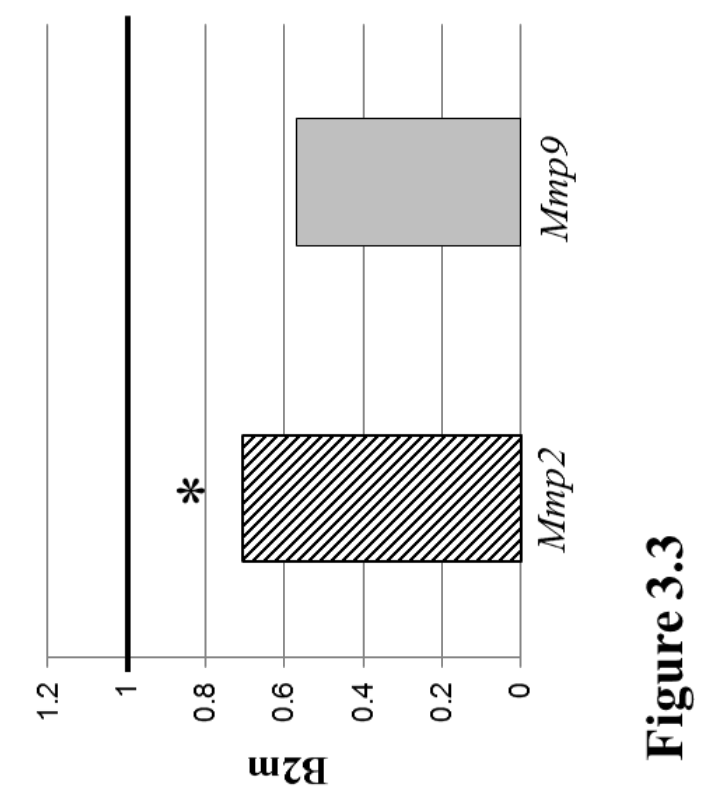

шо.у әэиә.әу!! рІо 
Figure 3.3. Quantitative RT-PCR assessment of the expression of genes potentially involved in inward remodeling in offspring thoracic aortas. All gene expressions were normalized to the expression of $B 2 m\left(\Delta C_{T}\right)$ and then averaged within the respective groups. A. The relative difference in cycle thresholds $(\Delta \Delta \mathrm{CT})$ between the HF and LF treatment groups for $M m p 2$ and Mmp9. B. The relative difference $\Delta \Delta \mathrm{CT}$ between females and males for Mmp2 and Timp1. The error bars represent the S.E.M. The fold differences between treatments groups for each gene analyzed were then calculated. $\mathbf{C}=$ fold difference calculation of data presented in $\mathbf{A}$. $\mathbf{D}=$ fold difference calculation of data presented in $\mathbf{B}$. The solid black line across " 1 " demarcates "LF" for the two bars in $\mathbf{C}$ (diet effect) and "males” in D (sex effect). Mmp2 = diagonal line bar; $M m p 9$ = gray bar; Timp1 = black bar. MMp2 - HF vs. LF, females vs. males; $\mathrm{n}=24$ and 29, $\mathrm{n}=24$ and 29, respectively. Mmp9 - HF vs. LF n=24 and 29, respectively. Timp1 - females vs. males; $\mathrm{n}=24$ and 29, respectively. All differences are significant at $\mathrm{p}<0.05$, except for the asterisk $(*)$ which represents a trend at $\mathrm{p}<0.1$. LF=consumption of maintenance chow by mothers and offspring. $\mathrm{HF}=$ maternal and offspring consumption of a diet with $24 \%$ fat and $17.5 \%$ high fructose corn syrup. $\Delta \mathrm{C}_{\mathrm{T}}=$ change in cycle threshold. $B 2 \mathrm{~m}=$ beta $2-$ microglobulin. 
increased intracellular ROS in mesenteric resistance arteries ( $\mathrm{p}=0.05 ; 45.2 \pm 2.47$ and $37.67 \pm 1.97$, respectively; Fig. 3.4B-C2). There was no significant difference in intracellular ROS between the HF and LF groups ( $\mathrm{p}>0.05 ; 41.96 \pm 2.35$ and $40.94 \pm 2.27$, respectively) or between the males and females ( $>0.05$; $39.57 \pm 2.08$ and $43.64 \pm 2.57$, respectively). Further, we did not observe a significant difference in the oxidation level of DHE outside the cells indicating that extracellular ROS did not differ in the mesenteric resistance arteries between treatment groups ( $>>0.05$, data not shown).

2-OH-Et and Et as measured by HPLC in the extracellular solution containing DHE are a measure of extracellular superoxide $\left(\mathrm{O}_{2}{ }^{-}\right)$production and non-specific ROS production, respectively. Intracellular ROS did not correlate significantly with either extracellular 2-OH-Et or Et (p>0.05, data not shown). When extracellular 2-OH-Et was increased, Et was also increased ( $<<0.0001$; Table 3.2). Extracellular 2-OH-Et was negatively correlated with the differences in cycle threshold of Mmp2, Mmp9, Timp1 and Nox2 ( $<<0.01, \mathrm{p}<0.01, \mathrm{p}<0.01$ and $\mathrm{p}<0.02$, respectively; Table 3.2), meaning that as the expression of each of the four genes was increased, extracellular superoxide was also increased. Extracellular Et was negatively correlated with the differences in cycle threshold of all of the genes analyzed (Table 3.2). Also, there was a negative correlation between extracellular 2-OH-Et and MAP (Table 3.2).

\subsection{Discussion}

Both ART (Ceelen et al. 2007, Belva et al. 2012) and maternal diet-induced obesity (Samuelsson et al. 2008, Nelson et al. 2010, Morandi et al. 2012) have been shown to produce offspring with increased body weight or increased adiposity. Our 


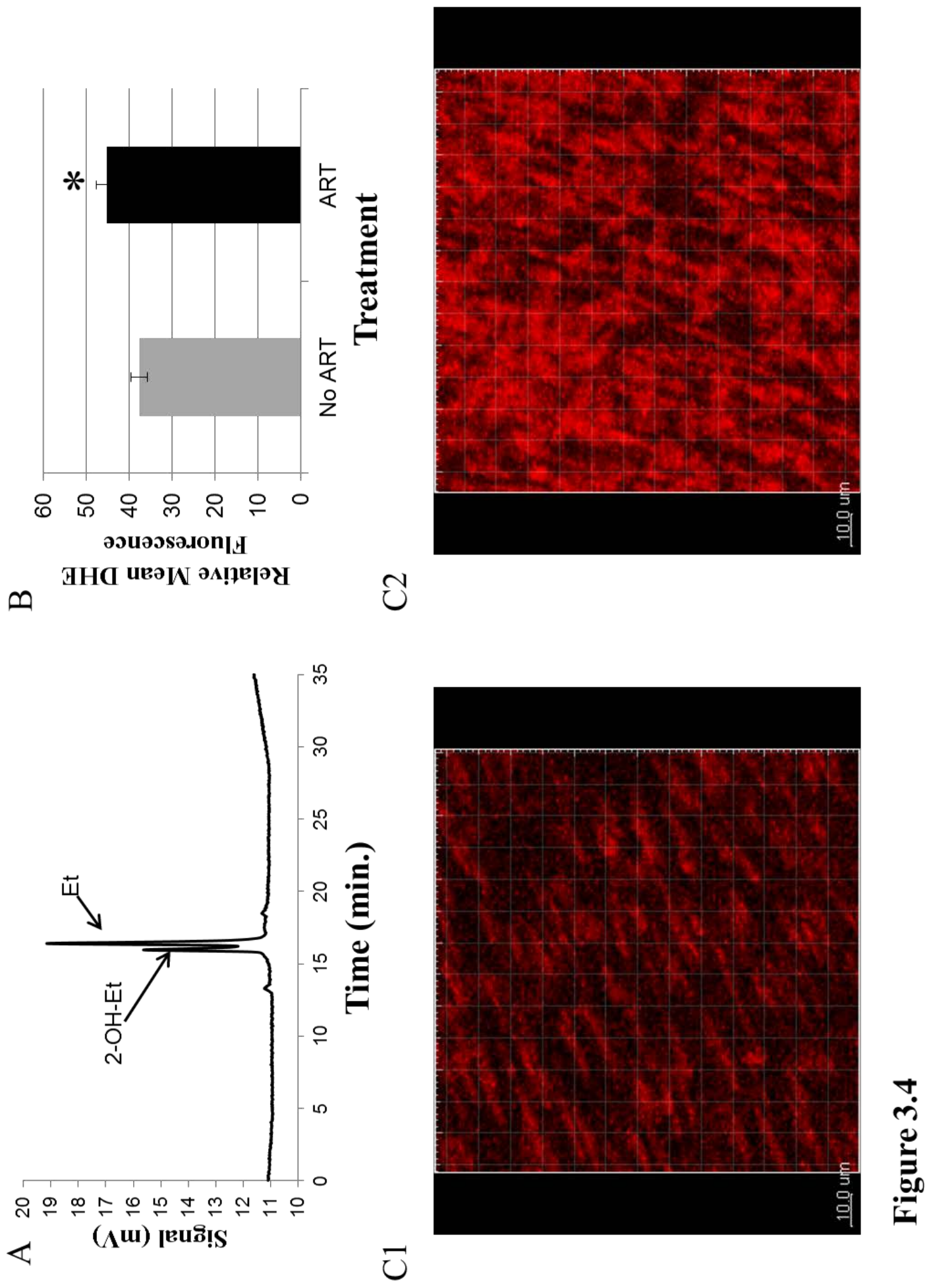


Figure 3.4. Detection of extracellular and intracellular ROS in mesenteric resistance arteries of offspring. A. Two fluorescent byproducts of dihydroethidium (DHE) were detected by high performance liquid chromatography (HPLC) in offspring (55 \pm 0.5 days) mesenteric resistance arteries. Resistance arteries were incubated with 50 $\mu \mathrm{M}$ DHE for one hour. 2-hydroxyethidium (2-OH-Et; specific byproduct of superoxide and DHE) eluted at $16.19 \pm 0.03 \mathrm{~min}$ and the ethidium (Et; less specific byproduct) eluted at $16.61 \pm 0.03 \mathrm{~min}$. Areas under each curve were determined using the Empower program (Waters, Build \#1154) and were normalized to the concentration of protein in each vessel before comparisons between treatments. Protein amounts were with a Micro BCA $^{\mathrm{TM}}$ Protein Assay Kit. B. Determination of levels of intracellular ROS in offspring mesenteric resistance arteries using confocal microscopy for relative DHE fluorescence. Intracellular DHE fluorescence (red) was measured by confocal microscopy. Gain was set at $800 \mathrm{~V}$. Data represent the group average \pm S.E.M. of DHE fluorescence over the average of two areas of the mesenteric resistance arteries for offspring conceived naturally (No ART; gray, $n=32$ ) or with the use of ART (ART; black, n=31). C. Shown is a confocal micrograph of a longitudinal section of a mesenteric resistance artery from a naturally conceived LF male (C.1) and an ART conceived LF male (C.2). Red fluorescence results from the oxidation of DHE. Fluorescence was quantified with the Imaris 7.6.1 software. ART = assisted reproductive technologies. DHE = dihydroethidium. ROS $=$ reactive oxygen species. ${ }^{*} \mathrm{p}=0.056$. 
study supported these previous findings, as both ART and diet affected offspring body weight. Maternal and offspring consumption of a HF diet resulted in offspring with higher body weight than the LF controls, and this effect persisted and increased from weaning (22 days) until sacrifice (55 \pm 0.5 days). However, the effect of ART that we observed on increased body weight in the offspring decreased over time after weaning. This is similar to findings from a previous study (Scott et al. 2010), where female mouse offspring conceived using IVF had increased body weight at three weeks of age when compared to no ART controls, but not from four weeks of age until eight weeks of age. Further, one retrospective ART study in humans showed increases in child adiposity rather than body weight (Ceelen et al. 2007), while the other showed increases in adiposity and body weight (Belva et al. 2012). However, the study by Belva et al. (2012) involved intracytoplasmic sperm injection (ICSI) which is a more invasive ART procedure compared to those used in our study. Future studies examining the effect of ART on offspring should look at adiposity in addition to body weight and also the type of ART procedure used to further clarify this issue.

Maternal and offspring diet had a greater effect on body weight in the male offspring compared to females. Males from the HF group weighed significantly more when compared to the LF males than the HF females did when compared to LF females. This finding is supported by previous research that exhibited pronounced adverse metabolic outcomes in males born to an obese mother (Torrens et al. 2012, Magliano et al. 2013). To our knowledge, no studies have previously been performed to examine the effect of ART and a HF developmental environment together on juvenile offspring body weight. Our study did not show a significant ART and diet interaction, suggesting that 
the adverse effects on offspring body weight from these two environments are not amplified by the combination of ART and HF diet consumption, at least at the age examined.

It has previously been reported that increased body weight can predispose an individual to adverse cardiovascular outcomes including hypertension and an increased risk for CVD (NIH 2012, Iguchi et al. 2013, Simoes-Silva et al. 2013). Data from our study support this previous research. First, males had increased body weight when compared to females from weaning until sacrifice, and this was accompanied by an increased MAP in males compared to females at sacrifice. The difference in MAP between males and females is also supported by previous research showing that estrogens can have a protective effect on blood pressure response [reviewed in (Xue et al. 2013)]. Further, MAP had a positive overall correlation with body weight at sacrifice, showing that individuals who were heavier had higher MAP.

Previous research has also shown increased blood pressure in offspring born to mothers consuming a high fat diet (Samuelsson et al. 2008, Torrens et al. 2012). Results from our study support these findings, as HF diet consumption (both by the dam and the offspring) resulted in increased MAP in the offspring.

In humans, an association has previously been made between the use of ART and increased systolic and diastolic blood pressure in children (Ceelen et al. 2008, Sakka et al. 2010). These retrospective studies involved children at ages from eight to 18 years old (Ceelen et al. 2008) and from four to 14 years old (Sakka et al. 2010) which we expect are comparable to the seven weeks of age juvenile mice that we studied. Further, an effect of ART on offspring blood pressure has also been observed in mice (Watkins et 
al. 2007). Our study, however, did not show increased MAP due to ART. There are several differences between the Watkins et al. (2007) work and ours that could partially explain the difference in results. For example, in our study we studied juvenile mice ( 7 weeks of age) while they used older mice (i.e. 15 and 21 weeks of age). In addition, the strain of mice used varied between studies [NSA (CF1) females and B6D2F1/J males vs (CBA X C57/BL6)F1 females and MF1 males for our and their studies, respectively] and the embryo culture medium was different (Whitten’s Medium vs T6 Medium, for our and their studies, respectively). Finally our measurement of MAP combines both systolic and diastolic pressure, while in the study by Watkins et al. (2007) only differences in systolic blood pressure were measured.

Interestingly, while offspring who were exposed to an obesogenic diet had increased MAP, they had lower expression of Mmp2 and Mmp9 when compared to offspring in the LF group. This is counterintuitive to previous studies that have suggested MMP2 and MMP9 to be involved in vascular remodeling associated with hypertension (Lehoux et al. 2004, Martinez-Lemus et al. 2011). However, in the study by Lehoux et al. (2004) MMP9 was shown to be involved in outward remodeling rather than inward remodeling (Lehoux et al. 2004), so increased MMP9 has been suggested to initially provide a compensatory response to increased pressure (Martinez-Lemus \& Galinanes 2011). Therefore, it is possible that the decreased Mmp9 expression found in the offspring with increased MAP is representative of a decreased ability of these offspring to compensate for their increased MAP. It should be noted that our interpretation of the results are based on measurements of signal for gene expression of 
the enzymes. Future experiments will determine whether protein expression and enzymatic activity align with our current findings.

Further, while males had increased MAP compared to females, they displayed decreased expression of Mmp2. However, they also displayed decreased expression of the inhibitor Timp1. Increased Timp1 expression has previously been shown to inhibit the activity of MMP2 and MMP9 (Zacchigna et al. 2004); therefore, it is possible that decreased Timp1 expression in the males is allowing for more MMP2 and MMP9 activity when compared to the females.

An ART effect was observed with levels of intracellular ROS in the offspring mesenteric resistance arteries. Superovulation, embryo culture and embryo transfer were used in this experiment in order to simulate three of the procedures commonly used in human ART. In this initial study, we chose to use Whitten's Medium to culture the embryos as this medium is known to be suboptimal for embryo development even though it is adequate to support development to full term (Ecker et al. 2004, Sommovilla et al. 2005). Future studies will determine if similar effects are observed with various culture conditions. Offspring who were conceived using ART had increased levels of ROS in their mesenteric resistance arteries when compared to the offspring who were conceived naturally. While this increase was not accompanied by a significant change in MAP, this could predispose the offspring to greater vascular dysfunction and remodeling, as increased levels of ROS have been associated with reduced bioavailability of NO and vascular remodeling (Katakam et al. 2005, Martinez-Lemus et al. 2011). The increased levels of intracellular ROS in the ART group also occurred without a significant change in Nox2 expression. This could be an artifact of the different tissues used for ROS 
quantification and gene expression analysis. Alternatively, the increased ROS levels could be due to other producers of ROS [i.e. NOX1, NOX4, xanthine oxidase and nitric oxide synthase; (Cai et al. 2003, Bedard \& Krause 2007, Demarco et al. 2010)]. Extracellular ROS levels were not significantly affected by any of the treatments.

We chose to study ROS levels in the resistance arteries, as the microvasculature is where most of the inward remodeling has been implicated in hypertension (Korsgaard et al. 1993). Due to limited amounts of tissue from the microvasculature, we performed gene expression analysis in the aorta. Dysfunction in the aorta has also been implicated in adverse cardiovascular health in rodents, and studies have been performed examining the MMPs and TIMPs in the aorta (Allaire et al. 1998, Khan et al. 2012). While we acknowledge that the aorta (a large conduit vessel) and the resistance arteries (resistance vessels) are not the same, and we cannot make strong causative conclusions in our findings between the two tissues, we can still use our findings as possible markers of adverse cardiovascular health in the offspring. Previous studies have also used both large conduit vessels and small resistance vessels to examine cardiovascular health in rodents (Beyer et al. 2008, Davidson et al. 2010, Sakurada et al. 2010, Agbor et al. 2012). In summary, our study has shown an effect of ART on offspring body weight at weaning in a rodent model. We have also shown an effect of the use of ART on the level of ROS in mesenteric resistance arteries in the offspring. Maternal and offspring consumption of a HF diet resulted in increased body weight, MAP and misregulated gene expression in the offspring aortas. We further found a relationship between elevated extracellular ROS from mesenteric resistance arteries and increased gene expression of Nox2, Mmp2, Mmp9, and Timp1 in the thoracic aorta. Knowledge is lacking on what 
effect the combination of ART and HF diet have on offspring body weight and cardiovascular health, but our data suggest that the two developmental environments do not have an additive effect on body weight, MAP, gene expression in thoracic aorta and ROS in mesenteric resistance arteries in juvenile mice. The experimental design used in the present study does not allow us to differentiate if the adverse cardiovascular outcomes observed were the result of exposure to an obesogenic diet prenatally or postnatally. Future research will examine this question. Further, as CVD in humans often does not present until adulthood (Jousilahti et al. 1999), follow-up studies will also determine if an increased risk for adverse cardiovascular outcomes resulting from ART or obesogenic diet alone or in combination is evident later in life.

\subsection{Declaration of interest}

None declared.

\subsection{Funding}

ALS is funded by a Miller Fellowship granted by MU Division of Animal Sciences. RMR is funded by F21C. LAM-L is funded by grant R01 HL088105 from the National Heart, Lung and Blood Institute.

\subsection{Acknowledgements}

We would like to thank Matthew Sepúlveda from the Rivera laboratory for his valuable assistance with embryo transfer surgeries, and Guiling Zhao from the Martínez-Lemus laboratory for her help with isolation of the mesenteric resistance arteries. 


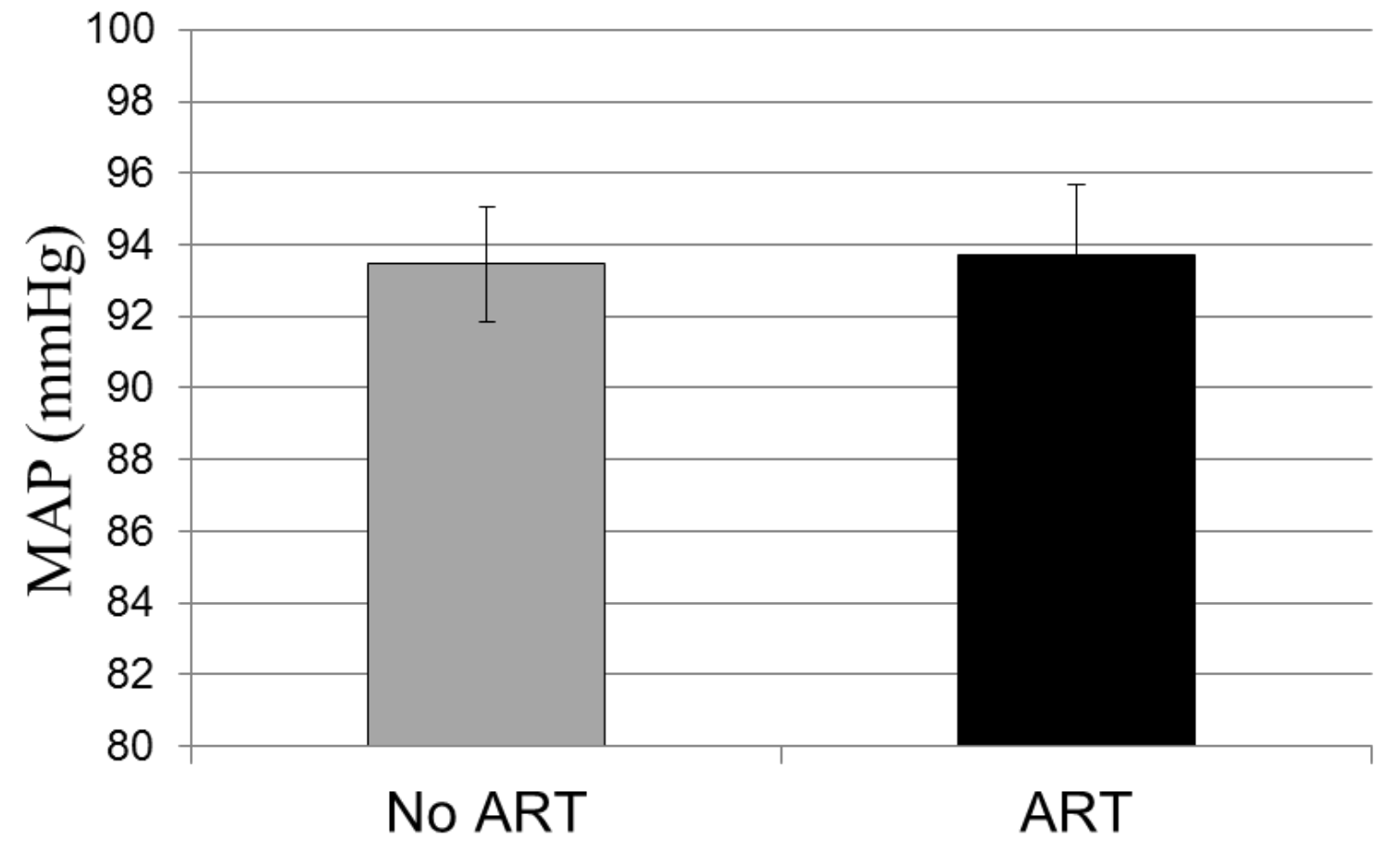

Supplementary Figure 3.1. Mean arterial pressure (MAP) in ART offspring and no ART controls at sacrifice. Carotid artery catheterization was performed to measure MAP as a representation of blood pressure in the offspring immediately prior to sacrifice ( $55 \pm 0.5$ days). Group averages were compared between the naturally-conceived controls (No ART; grey bar; $\mathrm{n}=30$ ) and the ART-conceived offspring (ART; black bar; $\mathrm{n}=29$ ). Data are presented as mean \pm the S.E.M. 
A

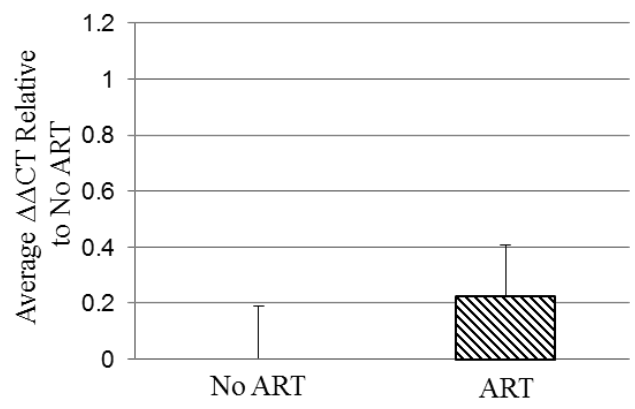

$\mathrm{C}$

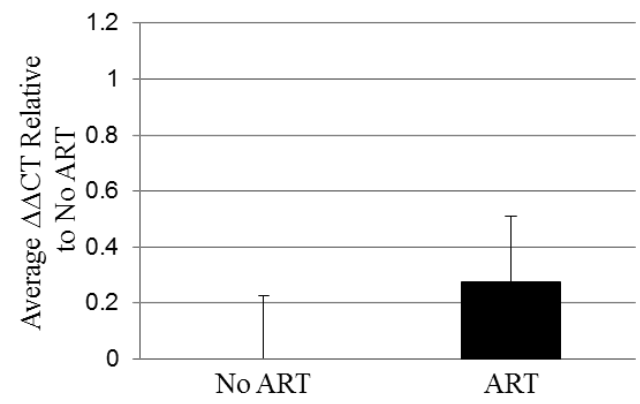

$\mathrm{E}$

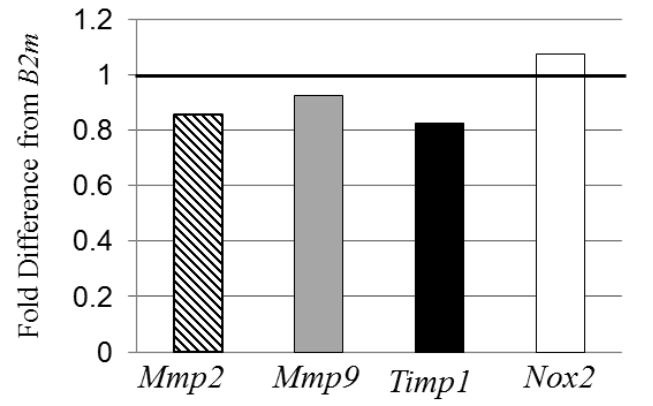

$\mathrm{B}$

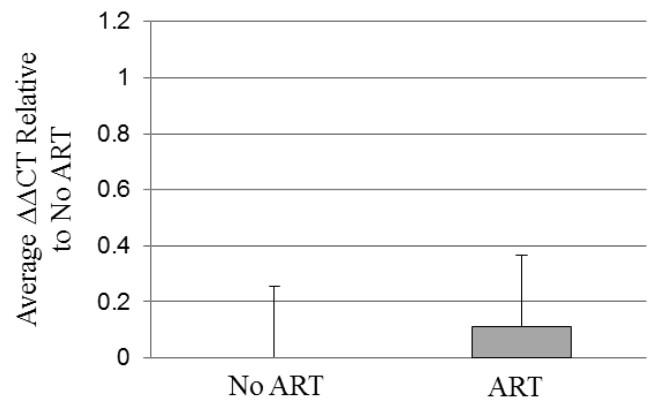

$\mathrm{D}$

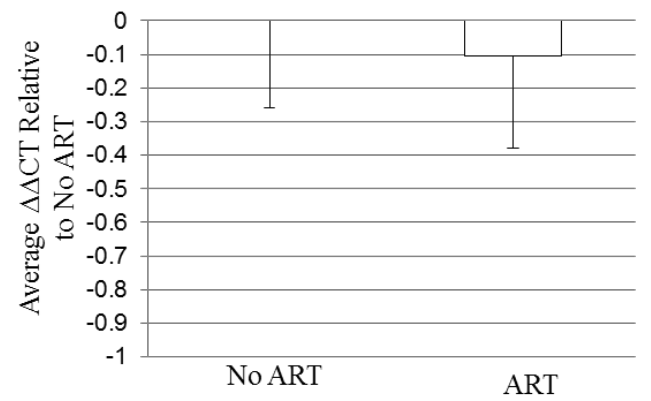

Supplementary Figure 3.2. Quantitative RT-PCR assessment of the expression of genes potentially involved in inward remodeling in thoracic aortas of ART offspring and no ART controls. All gene expressions were normalized to the expression of $B 2 m$ and then averaged within the respective groups. The relative difference in cycle thresholds $(\triangle \Delta C T)$ between the ART $(n=32)$ and no ART $(n=21)$ treatment groups for Mmp2 (A), Mmp9 (B), Timp1 (C) and Nox2 (D). The no ART group serves as the control. A, B, $\mathbf{C}$ and $\mathbf{D}=$ mean \pm S.E.M. The fold differences between treatments groups for each gene analyzed were then calculated. The solid black line across " 1 " demarcates "No ART" for the four bars in $\mathbf{E}$. 


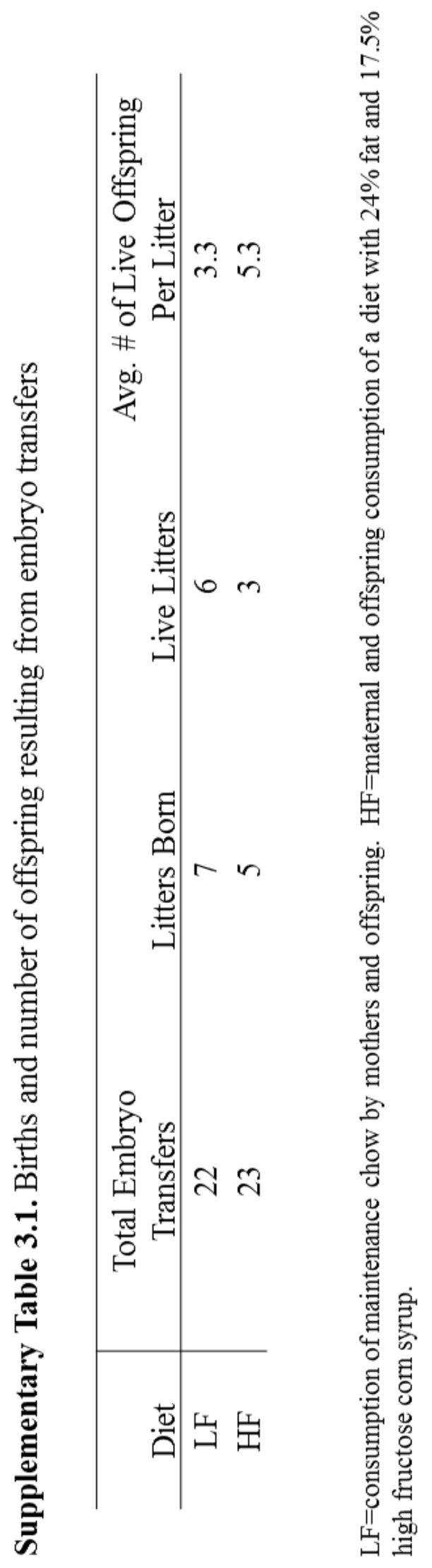




\section{CHAPTER 4}

\section{General Discussion}

The DOHaD hypothesis has revealed that the maternal environment in which fetal development occurs can affect the offspring's subsequent disease state in adulthood (Barker et al. 1989, Gillman et al. 2007). Two suboptimal maternal environments for fetal development are those that result from the use of ART, or that result from maternal obesity or consumption of a HF diet. The use of ART is on the rise with infertility affecting approximately 10\% of couples (CDC 2012). Furthermore, obesity is also becoming more common, and it is estimated that by 2030, $60 \%$ of adults will be overweight or obese (Kelly et al. 2008). Both of these increasingly common maternal environments can result in adverse cardiovascular and metabolic outcomes in the offspring (Ceelen et al. 2007, Watkins et al. 2007, Ceelen et al. 2008, Nelson et al. 2010, Torrens et al. 2012). However, little is known about what effect these two environments can have on the offspring when they occur simultaneously. Knowledge on the combined effects ART and maternal obesity would be beneficial because they often coincide. Obese women have increased rates of infertility; therefore, the use of ART by obese women is increasing (Robker 2008).

CVD is the number one cause of death world-wide (WHO 2013b). However, the mechanisms that result in the development of CVD are largely unknown. With the DOHaD hypothesis and the ability of the aforementioned maternal environments to adversely affect cardiovascular health, we wanted to use ART and consumption of a HF 
diet in a mouse model to examine the effects on possible markers of adverse cardiovascular health in the offspring.

Further with the increasing prevalence of obese women pursuing ART to conceive, we wanted to examine the effects of ART and consumption of a HF diet combined. To represent the use of ART in our model, we used superovulation, embryo culture in Whitten Medium, and embryo transfer. For the effect of diet, we fed females a diet with $24 \%$ fat and $17.5 \%$ high fructose corn syrup during oocyte maturation ( 3 weeks), pregnancy, lactation and weaning. For the group combining ART and HF diet, embryos were transferred into superovulated pseudopregnant recipients consuming the same diet as the embryo donors. All offspring were maintained on the same diet as their mother.

We first measured body weight weekly for the offspring, as increased body weight can increase the risk for CVD (NIH 2012). Hypertension has also been associated with increased risk for CVD (NIH 2012), so we examined MAP in the offspring at sacrifice. We further examined the expression of genes that have been implicated in vascular remodeling and adverse cardiovascular health (Mmp2, Mmp9, Timp1, and Nox2) in offspring aortas at sacrifice. The last measure of cardiovascular health that we chose for our study was levels of ROS which have been previously shown in our lab to increase activity of MMPs in inward vascular remodeling in rat cremaster arterioles (MartinezLemus et al. 2011). We measured ROS both intracellularly and extracellularly in offspring mesenteric arterioles.

We first wanted to examine the effect of exposure to an obesogenic diet (HF) on the offspring cardiovascular health (Results summarized in Fig. 4.1A). An association 
has been found in both human and mouse models between exposure to maternal high fat diet consumption while in utero and increased offspring body weight (Samuelsson et al. 2008, Nelson et al. 2010, Morandi et al. 2012). Exposure to a high fat in utero environment has also been associated with adverse cardiovascular outcomes in the offspring including increased blood pressure (Samuelsson et al. 2008, Torrens et al. 2012). Our data support the previous findings, as offspring exposed to an obesogenic diet were heavier from weaning until sacrifice, and also had a higher MAP when compared to the low fat diet controls. MAP also had a positive correlation with body weight at sacrifice (SacBW).

Based on previous research, we hypothesized that an increase in MAP could be associated with the MMPs, TIMPs and NOX-produced ROS. Previous findings in our lab and others have implicated MMP2 and MMP9 in the vascular remodeling associated with hypertension (Lehoux et al. 2004, Martinez-Lemus et al. 2011). However, increased MAP was not correlated with the expression of Mmp2 or Mmp9. Future studies should examine the activity of the MMPs in case the activity is increased without a change in expression. Alternatively, this could mean that there are other pathways involved in the increase in MAP.

ROS are involved in activating the MMPs in vascular remodeling (MartinezLemus et al. 2011); therefore, we were not surprised that our study showed that as extracellular ROS decreased the expression of the MMPs decreased as well. NOX (i.e. NOX2) is the main producer of ROS in the vasculature (Cai et al. 2003), and we found that as NOX2 expression decreased, the production of extracellular ROS also decreased. 

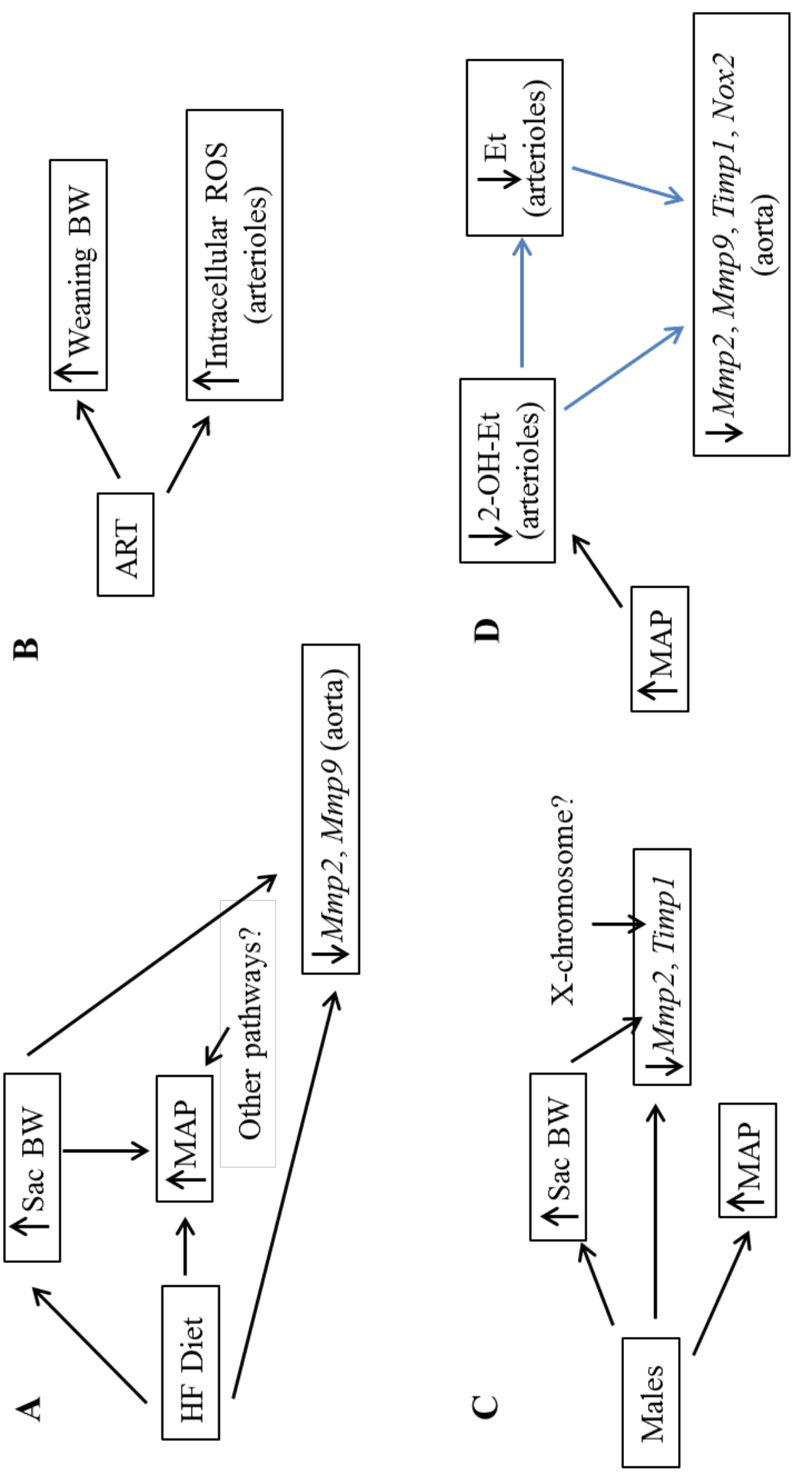

굼 
Figure 4.1 Summary of significant effects on offspring cardiovascular health. The effects of maternal and offspring consumption of a HF diet are summarized in A. Exposure to an obesogenic diet (HF diet) resulted in increased body weight at sacrifice (SacBW; $55 \pm 0.5$ days) as well as an increase in MAP in the offspring, indicative of adversely affected cardiovascular health. The HF group also had decreased expression of Mmp9 and a trend for decreased Mmp2 expression, which we cannot explain at this time. An increase in SacBW correlated with increased MAP and decreased Mmp2 expression and a trend toward decreased Mmp9 expression. There was no correlation between MAP and expression of $M m p 2$ or $M m p 9$, so we can speculate that other pathways might be involved to increase MAP. B summarizes the significant effects of ART on offspring cardiovascular health. Offspring body weight in the ART group at weaning (22 days) was increased compared to the No ART controls, but this effect diminished over time. However, intracellular ROS were increased in the ART-conceived offspring mesenteric arterioles at sacrifice indicating oxidative stress. This could potentially lead to increased vascular remodeling or endothelial dysfunction. $\mathbf{C}$ shows the gender effect on offspring cardiovascular health. Males had increased MAP and SacBW compared to females, as well as decreased Mmp2 and Timp1 expression. We speculate that decreased expression of Timp1 in males could be due to the ability of Timp1 to escape X-chromosome inactivation in females, leading to increased expression in the females. $\mathbf{D}$ represents correlations between MAP and extracellular 2-OH-Et, and between 2-OH-Et and gene expression. An increase in MAP correlates with an increase in 2-OH-Et, without a change in Nox2 expression. Extracellular 2-OH-Et and Et are positively correlated. Further, they both correlate positively with expression of all of the genes analyzed. Blue lines= the association between 2-OH-Et and Et, and between both 2-OH-Et and Et with gene expression. The blue lines serve to separate these associations from the association between MAP and 2-OH-Et, as MAP is not significantly correlated with either $\mathrm{Et}$ or the gene expressions. 2-OH-Et=2-hydroxyethidium. $\mathrm{Et}=$ ethidium. $\mathrm{MAP}=$ mean arterial pressure. 
A finding that is more puzzling is that with increased SacBW the expression of Mmp2 was lower, even though increased SacBW was positively correlated with increased MAP. While we did not analyze the correlation between SacBW and Mmp2 expression in males and females separately, it is possible that the correlation between SacBW and lower Mmp2 expression was driven by the males because they weighed more and had less Mmp2 expression than the females. However, we cannot prove causality based on this speculation. Further, exposure to an obesogenic diet resulted in increased MAP accompanied by a lower Mmp9 expression. MMP9 has been proposed to be involved in a compensation mechanism to induce outward remodeling in response to high vascular pressure (Lehoux et al. 2004, Martinez-Lemus \& Galinanes 2011), but it is possible that the lower Mmp9 in the HF group with higher blood pressure is showing a failed ability to compensate for the higher blood pressure.

Secondly, we wanted to examine how ART could affect the cardiovascular health in the offspring (results demonstrated in Fig. 4.1B). Previous research has shown increased body weight or adiposity with the use of ART in both human and mouse models (Ceelen et al. 2007, Scott et al. 2010, Belva et al. 2012). In our study, we found that offspring conceived using ART were heavier at weaning (Weaning BW) than the no ART controls, but this effect of ART on body weight diminished over time. This is similar to the finding in Scott et al. (2010) where female mice conceived with the use of IVF were heavier at three weeks of age when compared to no ART controls, but were no longer heavier from four weeks until eight weeks of age (Scott et al. 2010).

Previous studies have shown increased oxidative stress with the use of ART at the level of the ovary, oocyte and embryo (Chao et al. 2005, Ge et al. 2012). We further 
showed an increase in intracellular ROS in the second order mesenteric arterioles from our ART conceived offspring when compared to those that were naturally conceived. This increase was not accompanied by an increase in Nox2 expression in the offspring. One possible explanation for this is that ROS can be produced by other enzymes [i.e. NOX1, NOX4, xanthine oxidase, and nitric oxide synthase; (Cai et al. 2003, Bedard \& Krause 2007, Demarco et al. 2010)]. An increase in intracellular ROS without an increase in Nox2 expression could also be an artifact of using arterioles for ROS measurement and aorta for gene expression analysis.

Determining the effect of gender was not one of the main goals of our study; however, we could not ignore that gender might have an effect (results summarized in Fig. 4.1C). Males had increased body weight when compared to females, and this was accompanied by an increase in MAP. This result could be an effect of the positive correlation between MAP and SacBW mentioned earlier. Alternatively, the effect of gender on MAP could be a true gender effect, as it has previously been shown that estrogen in females can have a protective effect on blood pressure response [reviewed in (Xue et al. 2013)]. Males also had decreased expression of Mmp2 and Timp1. The lower expression of Mmp2 in males could be due to the correlation between increased SacBW and lower Mmp2 expression mentioned previously. We can speculate that the lower expression of Timp1 might be a true gender effect because Timp1 is located on the $\mathrm{X}$-chromosome and has the ability to escape X-chromosome inactivation (Anderson \& Brown 1999). X-chromosome inactivation is a mechanism leading to equal expression of $\mathrm{X}$-linked genes between males and females, so if the Timp1 is escaping this inactivation, it could explain why females would have higher expression than males. 
As increased levels of ROS can increase the expression of the MMPs (Zhang et al. 2013), it is possible that the negative correlation we observed between 2-OH-Et and Et with the difference in cycle threshold of the MMPs was due to a ROS-dependent increase in gene expression (Fig. 4.1D). Further, $\mathrm{NOX}$ (i.e. $\mathrm{NOX} 2$ ) is the main producer of $\mathrm{O}_{2}{ }^{-}$in the vasculature (Cai et al. 2003), so it is not surprising that we observed increased levels of 2-OH-Et and a trend toward increased levels of Et (from downstream products of $\mathrm{O}_{2}{ }^{-}$) when Nox2 expression was increased.

Interestingly, extracellular levels of 2-OH-Et (representative of $\mathrm{O}_{2}{ }^{-}$levels) were decreased with an increase in MAP without a significant decrease in Nox2 expression or the production of downstream products of $\mathrm{O}_{2}^{-}$(represented by Et). We can speculate that the offspring with increased MAP might be compensating in a way that would reduce superoxide levels. This could be accomplished by decreased activity of NOX2 as NOX is involved in $\mathrm{O}_{2}{ }^{-}$production in the vasculature, or perhaps by decreasing the bioavailability of $\mathrm{O}_{2}{ }^{-}$with increased SOD (Li et al. 2001) or increased NO production which would favor the production of peroxynitrite (Frears et al. 1996). Future studies should look at the levels of scavengers such as SOD or producers of NO such as nitric oxide synthase to see if this compensation might be occurring.

In summary, the findings from this study have provided a gateway for future research. Previous studies combined with our study show that ART and consumption of an obesogenic diet can adversely affect the offspring. These effects range from increased oxidative damage and misregulated gene expression, to metabolic differences in the children conceived using ART. While we did not observe a combined effect of ART and consumption of an obesogenic diet in juvenile offspring cardiovascular health $(\sim 7$ 
weeks), we did observe effects of each environment independently on markers of adverse cardiovascular health. Our study was performed in mice so the findings can only be suggestive to humans, but our results together with other animal and human studies may be used to caution obese individuals planning to conceive or individuals considering assisted reproduction of possible outcomes. 


\section{BIBLIOGRAPHY}

Abramczuk J, Solter D \& Koprowski H. 1977 The beneficial effect of EDTA on development of mouse one-cell embryos in chemically defined medium. Dev Biol. 61 378-383.

ADA 2013 Diagnosis and classification of diabetes mellitus. Diabetes Care 36 Suppl 1 S67-74.

Agbor LN, Walsh MT, Boberg JR \& Walker MK 2012 Elevated blood pressure in cytochrome P4501A1 knockout mice is associated with reduced vasodilation to omega-3 polyunsaturated fatty acids. Toxicol Appl Pharmacol 264 351-360.

AHA. 2013. Atherosclerosis and stroke. Retrieved July 23, 2013 from http://www.strokeassociation.org/STROKEORG/LifeAfterStroke/HealthyLiving AfterStroke/UnderstandingRiskyConditions/Atherosclerosis-andStroke_UCM_310426_Article.jsp.

Ajayi AA, Elliott HL \& Reid JL 1986 The pharmacodynamics and dose-response relationships of the angiotensin converting enzyme inhibitor, cilazapril, in essential hypertension. Br J Clin Pharmacol 22 167-175.

Alberti KG \& Zimmet PZ 1998 Definition, diagnosis and classification of diabetes mellitus and its complications. Part 1: diagnosis and classification of diabetes mellitus provisional report of a WHO consultation. Diabet Med 15 539-553.

Allaire E, Forough R, Clowes M, Starcher B \& Clowes AW 1998 Local overexpression of TIMP-1 prevents aortic aneurysm degeneration and rupture in a rat model. J Clin Invest 102 1413-1420.

Allan JA, Docherty AJ, Barker PJ, Huskisson NS, Reynolds JJ \& Murphy G 1995 Binding of gelatinases A and B to type-I collagen and other matrix components. Biochem J 309 ( Pt 1) 299-306.

Almamun M. 2011. Size-dependent acquisition of global DNA methylation in oocytes is altered by hormonal stimulation. Master’s Thesis, University of Missouri, Columbia. 
Alzamendi A, Castrogiovanni D, Gaillard RC, Spinedi E \& Giovambattista A 2010 Increased male offspring's risk of metabolic-neuroendocrine dysfunction and overweight after fructose-rich diet intake by the lactating mother. Endocrinology 151 4214-4223.

Anderson CL \& Brown CJ 1999 Polymorphic X-chromosome inactivation of the human TIMP1 gene. Am J Hum Genet 65 699-708.

Anderson CL \& Brown CJ 2005 Epigenetic predisposition to expression of TIMP1 from the human inactive X chromosome. BMC Genet 648.

Anrather J, Racchumi G \& Iadecola C 2006 NF-kappaB regulates phagocytic NADPH oxidase by inducing the expression of gp91phox. J Biol Chem 281 5657-5667.

Antonaccio MJ, Rubin B, Horovitz ZP, Laffan RJ, Goldberg ME, High JP, Harris DN \& Zaidi I 1979 Effects of chronic treatment with captopril (SQ 14,225), an orally active inhibitor of angiotensin I-converting enzyme, in spontaneously hypertensive rats. Jpn J Pharmacol 29 285-294.

\section{Armitage JA, Pearce AD, Sinclair AJ, Vingrys AJ, Weisinger RS \& Weisinger HS} 2003 Increased blood pressure later in life may be associated with perinatal n-3 fatty acid deficiency. Lipids 38 459-464.

Armitage JA, Poston L \& Taylor PD 2008 Developmental origins of obesity and the metabolic syndrome: the role of maternal obesity. Front Horm Res 36 73-84.

Babior BM, Kipnes RS \& Curnutte JT 1973 Biological defense mechanisms. The production by leukocytes of superoxide, a potential bactericidal agent. J Clin Invest 52 741-744.

Bailey-Downs LC, Tucsek Z, Toth P, Sosnowska D, Gautam T, Sonntag WE, Csiszar A \& Ungvari Z 2013 Aging exacerbates obesity-induced oxidative stress and inflammation in perivascular adipose tissue in mice: a paracrine mechanism contributing to vascular redox dysregulation and inflammation. J Gerontol A Biol Sci Med Sci 68 780-792.

Balasubramanian P, Jagannathan L, Mahaley RE, Subramanian M, Gilbreath ET, Mohankumar PS \& Mohankumar SM 2012 High fat diet affects reproductive functions in female diet-induced obese and dietary resistant rats. $J$ Neuroendocrinol 24 748-755. 
Baldridge CW \& Gerard RW. 1932. The extra respiration of phagocytosis. Am J Physiol 103 235-236.

Baltz JM 2012 Media composition: salts and osmolality. Methods Mol Biol 912 61-80.

Barker DJ, Larsen G, Osmond C, Thornburg KL, Kajantie E \& Eriksson JG 2012 The placental origins of sudden cardiac death. Int J Epidemiol 41 1394-1399.

Barker DJ \& Osmond C 1986 Infant mortality, childhood nutrition, and ischaemic heart disease in England and Wales. Lancet 1 1077-1081.

Barker DJ, Osmond C, Kajantie E \& Eriksson JG 2009 Growth and chronic disease: findings in the Helsinki Birth Cohort. Ann Hum Biol 36 445-458.

Barker DJ, Winter PD, Osmond C, Margetts B \& Simmonds SJ 1989 Weight in infancy and death from ischaemic heart disease. Lancet 2 577-580.

Barrett KE, Barman SM, Boitano, S. \& Brooks, H. 2012 Ganong's Review of Medical Physiology, Twenty-Fourth Edition. New York, NY: The McGraw-Hill Companies.

Bayol SA, Simbi BH \& Stickland NC 2005 A maternal cafeteria diet during gestation and lactation promotes adiposity and impairs skeletal muscle development and metabolism in rat offspring at weaning. J Physiol 567 951-961.

Becker-Follmann J, Gaa A, Bausch E, Natt E, Scherer G \& von Deimling O 1997 High-resolution mapping of a linkage group on mouse chromosome 8 conserved on human chromosome 16Q. Mamm Genome 8 172-177.

Bedard K \& Krause KH 2007 The NOX family of ROS-generating NADPH oxidases: physiology and pathophysiology. Physiol Rev 87 245-313.

Bellinger L, Lilley C \& Langley-Evans SC 2004 Prenatal exposure to a maternal lowprotein diet programmes a preference for high-fat foods in the young adult rat. $\mathrm{Br}$ J Nutr 92 513-520. 
Bellver J, Melo MA, Bosch E, Serra V, Remohi J \& Pellicer A 2007 Obesity and poor reproductive outcome: the potential role of the endometrium. Fertil Steril $\mathbf{8 8} 446$ 451.

Belva F, Painter R, Bonduelle M, Roelants M, Devroey P \& De Schepper J 2012 Are ICSI adolescents at risk for increased adiposity? Hum Reprod 27 257-264.

Bergman MR, Teerlink JR, Mahimkar R, Li L, Zhu BQ, Nguyen A, Dahi S, Karliner JS \& Lovett DH 2007 Cardiac matrix metalloproteinase-2 expression independently induces marked ventricular remodeling and systolic dysfunction. Am J Physiol Heart Circ Physiol 292 H1847-1860.

Bermejo-Alvarez P, Rosenfeld CS \& Roberts RM 2012 Effect of maternal obesity on estrous cyclicity, embryo development and blastocyst gene expression in a mouse model. Hum Reprod 27 3513-3522.

Beyer AM, Baumbach GL, Halabi CM, Modrick ML, Lynch CM, Gerhold TD, Ghoneim SM, de Lange WJ, Keen HL, Tsai YS, Maeda N, Sigmund CD \& Faraci FM 2008 Interference with PPARgamma signaling causes cerebral vascular dysfunction, hypertrophy, and remodeling. Hypertension 51 867-871.

Bhattacharya S, Campbell DM \& Liston WA 2007 Effect of Body Mass Index on pregnancy outcomes in nulliparous women delivering singleton babies. $B M C$ Public Health 7168.

Biggers JD 1998 Reflections on the culture of the preimplantation embryo. Int J Dev Biol 42 879-884.

Binder NK, Mitchell M \& Gardner DK 2012 Parental diet-induced obesity leads to retarded early mouse embryo development and altered carbohydrate utilisation by the blastocyst. Reprod Fertil Dev 24 804-812.

Bode W 1995 A helping hand for collagenases: the haemopexin-like domain. Structure 3 527-530.

Bode W, Gomis-Ruth FX \& Stockler W 1993 Astacins, serralysins, snake venom and matrix metalloproteinases exhibit identical zinc-binding environments (HEXXHXXGXXH and Met-turn) and topologies and should be grouped into a common family, the 'metzincins'. FEBS Lett 331 134-140. 
Boney CM, Verma A, Tucker R \& Vohr BR 2005 Metabolic syndrome in childhood: association with birth weight, maternal obesity, and gestational diabetes mellitus. Pediatrics 115 e290-296.

Bray GA, Nielsen SJ \& Popkin BM 2004 Consumption of high-fructose corn syrup in beverages may play a role in the epidemic of obesity. Am J Clin Nutr 79 537-543.

Brenseke B, Prater MR, Bahamonde J \& Gutierrez JC 2013 Current thoughts on maternal nutrition and fetal programming of the metabolic syndrome. $J$ Pregnancy 2013. Article ID 368461, 13 pages, 2013. doi:10.1155/2013/368461.

Briet M \& Schiffrin EL 2013 Treatment of arterial remodeling in essential hypertension. Curr Hypertens Rep 15 3-9.

Brooks AA, Johnson MR, Steer PJ, Pawson ME \& Abdalla HI 1995 Birth weight: nature or nurture? Early Hum Dev 42 29-35.

Brown AS \& Susser ES 2008 Prenatal nutritional deficiency and risk of adult schizophrenia. Schizophr Bull 34 1054-1063.

Brown AS, van Os J, Driessens C, Hoek HW, \& Susser ES. 2000 Further evidence of relation between prenatal famine and major affective disorder. Am J Psychiatry 157 190-195.

Buettner GR 2008 DETAPAC (DTPA), Aqueous Stock Solution. Retrieved July 23, 2013 from http://www.healthcare.uiowa.edu/corefacilities/esr/protocols/ detapac/DETAPAC-Protocol.pdf.

Burton GJ, Jauniaux E \& Watson AL 1999 Maternal arterial connections to the placental intervillous space during the first trimester of human pregnancy: the Boyd collection revisited. Am J Obstet Gynecol 181 718-724.

Cai H, Griendling KK \& Harrison DG 2003 The vascular NAD(P)H oxidases as therapeutic targets in cardiovascular diseases. Trends Pharmacol Sci 24 471-478.

Cai H \& Harrison DG 2000 Endothelial dysfunction in cardiovascular diseases: the role of oxidant stress. Circ Res 87 840-844. 
Cai W, Torreggiani M, Zhu L, Chen X, He JC, Striker GE \& Vlassara H 2010 AGER1 regulates endothelial cell NADPH oxidase-dependent oxidant stress via PKC-delta: implications for vascular disease. Am J Physiol Cell Physiol 298 C624-634.

Calkins K \& Devaskar SU 2011 Fetal origins of adult disease. Curr Probl Pediatr Adolesc Health Care 41 158-176.

Carroll D, Ginty AT, Painter RC, Roseboom TJ, Phillips AC \& de Rooij SR 2012 Systolic blood pressure reactions to acute stress are associated with future hypertension status in the Dutch Famine Birth Cohort Study. Int J Psychophysiol 85 270-273.

CDC. 2011. Aortic Aneurysm Fact Sheet. Retrieved July 23, 2013 from http://www.cdc.gov/dhdsp/data_statistics/fact_sheets/fs_aortic_aneurysm.htm.

CDC. 2012. 2010 Assisted Reproductive Technology Fertility Clinic Success Rates Report. Retrieved July 23, 2013 from http://www.cdc.gov/art/ ART2010/PDFs/ART_2010_National_Summary_Report.pdf.

CDC. 2013. Reproductive Health. Retroeved July 24, 2013 from http://www.cdc.gov/reproductivehealth/Infertility/.

Ceelen M, van Weissenbruch MM, Prein J, Smit JJ, Vermeiden JP, Spreeuwenberg M, van Leeuwen FE \& Delemarre-van de Waal HA 2009 Growth during infancy and early childhood in relation to blood pressure and body fat measures at age 8-18 years of IVF children and spontaneously conceived controls born to subfertile parents. Hum Reprod 24 2788-2795.

Ceelen M, van Weissenbruch MM, Roos JC, Vermeiden JP, van Leeuwen FE \& Delemarre-van de Waal HA 2007 Body composition in children and adolescents born after in vitro fertilization or spontaneous conception. J Clin Endocrinol Metab 92 3417-3423.

Ceelen M, van Weissenbruch MM, Vermeiden JP, van Leeuwen FE \& Delemarrevan de Waal HA 2008 Cardiometabolic differences in children born after in vitro fertilization: follow-up study. J Clin Endocrinol Metab 93 1682-1688. 
Chan SL \& Baumbach GL 2013 Nox2 deficiency prevents hypertension-induced vascular dysfunction and hypertrophy in cerebral arterioles. Int J Hypertens 2013. Article ID 793630, 8 pages, 2013. doi: 10.1155/2013/793630.

Chandra R, Federici S, Nemeth ZH, Horvath B, Pacher P, Hasko G, Deitch EA \& Spolarics Z 2011 Female X-chromosome mosaicism for NOX2 deficiency presents unique inflammatory phenotype and improves outcome in polymicrobial sepsis. J Immunol 186 6465-6473.

Chao HT, Lee SY, Lee HM, Liao TL, Wei YH \& Kao SH 2005 Repeated ovarian stimulations induce oxidative damage and mitochondrial DNA mutations in mouse ovaries. Ann N Y Acad Sci 1042 148-156.

Chen M, Norman RJ, Heilbronn LK 2011 Does in vitro fertilisation increase type 2 diabetes and cardiovascular risk? Current Diabetes Review 7 426-432.

Chen Z, Robbins KM, Wells KD \& Rivera RM 2013 Large offspring syndrome: A bovine model for the human loss-of-imprinting overgrowth syndrome BeckwithWiedemann. Epigenetics 8(6) 591-601.

Chesler NC, Ku DN \& Galis ZS 1999 Transmural pressure induces matrix-degrading activity in porcine arteries ex vivo. Am J Physiol 277 H2002-2009.

Chinen I, Shimabukuro M, Yamakawa K, Higa N, Matsuzaki T, Noguchi K, Ueda S, Sakanashi M \& Takasu N 2007 Vascular lipotoxicity: endothelial dysfunction via fatty-acid-induced reactive oxygen species overproduction in obese Zucker diabetic fatty rats. Endocrinology 148 160-165.

Choi MH, Cha SH, Park CW, Kim JY, Yang KM, Song IO, Koong MK, Kang IS \& Kim HO 2013 The effectiveness of earlier oocyte retrieval in the case of a premature luteinizing hormone surge on hCG day in in vitro fertilization-embryo transfer cycles. Clin Exp Reprod Med 40 90-94.

Chu SY, Callaghan WM, Kim SY, Schmid CH, Lau J, England LJ \& Dietz PM 2007 Maternal obesity and risk of gestational diabetes mellitus. Diabetes Care 30 20702076.

Chun TH, Inoue M, Morisaki H, Yamanaka I, Miyamoto Y, Okamura T, SatoKusubata K \& Weiss SJ 2010 Genetic link between obesity and MMP14dependent adipogenic collagen turnover. Diabetes 59 2484-2494. 
Cojocarui IM, Cojocaru M, Sapira V, Socoliuc G, Hertea C \& Paveliu S 2012 Changes in plasma matrix metalloproteinase-9 levels in patients with acute ischemic stroke. Rom J Intern Med 50 155-158.

Collier P, Watson CJ, Voon V, Phelan D, Jan A, Mak G, Martos R, Baugh JA, Ledwidge MT \& McDonald KM 2011 Can emerging biomarkers of myocardial remodelling identify asymptomatic hypertensive patients at risk for diastolic dysfunction and diastolic heart failure? Eur J Heart Fail 13 1087-1095.

Crabbe T, Willenbrock F, Eaton D, Hynds P, Carne AF, Murphy G \& Docherty AJ 1992 Biochemical characterization of matrilysin. Activation conforms to the stepwise mechanisms proposed for other matrix metalloproteinases. Biochemistry 31 8500-8507.

Czernichow S, Zanchetti A, Turnbull F, Barzi F, Ninomiya T, Kengne AP, Lambers Heerspink HJ, Perkovic V, Huxley R, Arima H, Patel A, Chalmers J, Woodward M, MacMahon S \& Neal B 2011 The effects of blood pressure reduction and of different blood pressure-lowering regimens on major cardiovascular events according to baseline blood pressure: meta-analysis of randomized trials. J Hypertens 29 4-16.

Davidson EP, Coppey LJ, Calcutt NA, Oltman CL \& Yorek MA 2010 Diet-induced obesity in Sprague-Dawley rats causes microvascular and neural dysfunction. Diabetes Metab Res Rev 26 306-318.

Davis OK \& Rosenwaks Z 2001 Superovulation strategies for assisted reproductive technologies. Semin Reprod Med 19 207-212.

Demarco VG, Whaley-Connell AT, Sowers JR, Habibi J \& Dellsperger KC 2010 Contribution of oxidative stress to pulmonary arterial hypertension. World $J$ Cardiol 2 316-324.

Denomme MM, Zhang L \& Mann MR 2011 Embryonic imprinting perturbations do not originate from superovulation-induced defects in DNA methylation acquisition. Fertil Steril 96(3) 734-738.

Depalo R, Garruti G, Totaro I, Panzarino M, Vacca MP, Giorgino F \& Selvaggi LE 2011 Oocyte morphological abnormalities in overweight women undergoing in vitro fertilization cycles. Gynecol Endocrinol 27 880-884. 
DeUgarte DA, DeUgarte CM \& Sahakian V 2010 Surrogate obesity negatively impacts pregnancy rates in third-party reproduction. Fertil Steril 93 1008-1010.

Di Cianni G, Miccoli R, Volpe L, Lencioni C, Ghio A, Giovannitti MG, Cuccuru I, Pellegrini G, Chatzianagnostou K, Boldrini A \& Del Prato S 2005 Maternal triglyceride levels and newborn weight in pregnant women with normal glucose tolerance. Diabet Med 22 21-25.

Dickey RP, Xiong X, Gee RE \& Pridjian G 2012 Effect of maternal height and weight on risk of preterm birth in singleton and twin births resulting from in vitro fertilization: a retrospective cohort study using the Society for Assisted Reproductive Technology Clinic Outcome Reporting System. Fertil Steril 97 349354.

Dokras A, Baredziak L, Blaine J, Syrop C, VanVoorhis BJ \& Sparks A 2006 Obstetric outcomes after in vitro fertilization in obese and morbidly obese women. Obstet Gynecol 108 61-69.

Dumollard R, Carroll J, Duchen MR, Campbell K \& Swann K 2009 Mitochondrial function and redox state in mammalian embryos. Seminars in Cell \& Developmental Biology 20 346-353.

Dumoulin JC, Land JA, Van Montfoort AP, Nelissen EC, Coonen E, Derhaag JG, Schreurs IL, Dunselman GA, Kester AD, Geraedts JP \& Evers JL 2010 Effect of in vitro culture of human embryos on birthweight of newborns. Hum Reprod 25 605-612.

Edwards RG, Steptoe PC \& Purdy JM 1980 Establishing full-term human pregnancies using cleaving embryos grown in vitro. Br J Obstet Gynaecol 87 737-756.

Eppig JJ, Wigglesworth K \& Pendola FL 2002 The mammalian oocyte orchestrates the rate of ovarian follicular development. Proc Natl Acad Sci U S A 992890 2894.

Eriksson JG, Forsen T, Tuomilehto J, Osmond C \& Barker DJ 2000 Early growth, adult income, and risk of stroke. Stroke 31 869-874.

Eriksson JG, Forsen T, Tuomilehto J, Osmond C \& Barker DJ 2001 Early growth and coronary heart disease in later life: longitudinal study. BMJ 322 949-953. 
Ertzeid G \& Storeng R 1992 Adverse effects of gonadotrophin treatment on pre- and postimplantation development in mice. J Reprod Fertil 96 649-655.

Ertzeid G \& Storeng R 2001 The impact of ovarian stimulation on implantation and fetal development in mice. Hum Reprod 16 221-225.

Eskandari MK, Vijungco JD, Flores A, Borensztajn J, Shively V \& Pearce WH 2005 Enhanced abdominal aortic aneurysm in TIMP-1-deficient mice. J Surg Res 123 289-293.

Fedorcsak P, Dale PO, Storeng R, Tanbo T \& Abyholm T 2001 The impact of obesity and insulin resistance on the outcome of IVF or ICSI in women with polycystic ovarian syndrome. Hum Reprod 16 1086-1091.

Fernandez-Patron C, Radomski MW \& Davidge ST 1999 Vascular matrix metalloproteinase-2 cleaves big endothelin-1 yielding a novel vasoconstrictor. Circ Res 85 906-911.

Fernandez-Sanchez A, Madrigal-Santillan E, Bautista M, Esquivel-Soto J, MoralesGonzalez A, Esquivel-Chirino C, Durante-Montiel I, Sanchez-Rivera G, Valadez-Vega C \& Morales-Gonzalez JA 2011 Inflammation, oxidative stress, and obesity. Int J Mol Sci 12 3117-3132.

Forsdahl A 1977 Are poor living conditions in childhood and adolescence an important risk factor for arteriosclerotic heart disease? Br J Prev Soc Med 31 91-95.

Fowden AL, Sibley C, Reik W \& Constancia M 2006 Imprinted genes, placental development and fetal growth. Horm Res 65 Suppl 3 50-58.

Fox SB \& Khong TY 1990 Lack of innervation of human umbilical cord. An immunohistological and histochemical study. Placenta 11 59-62.

Franco JG, Fernandes TP, Rocha CP, Calvino C, Pazos-Moura CC, Lisboa PC, Moura EG \& Trevenzoli IH 2012 Maternal high-fat diet induces obesity and adrenal and thyroid dysfunction in male rat offspring at weaning. J Physiol 590 5503-5518.

Franco Mdo C, Fortes ZB, Akamine EH, Kawamoto EM, Scavone C, de Britto LR, Muscara MN, Teixeira SA, Tostes RC, Carvalho MH \& Nigro D 2004 
Tetrahydrobiopterin improves endothelial dysfunction and vascular oxidative stress in microvessels of intrauterine undernourished rats. J Physiol 558 239-248.

Frantz C, Stewart KM \& Weaver VM 2010 The extracellular matrix at a glance. J Cell Sci 123 4195-4200.

Frears ER, Zhang Z, Blake DR, O'Connell JP \& Winyard PG 1996 Inactivation of tissue inhibitor of metalloproteinase-1 by peroxynitrite. FEBS Lett 381 21-24.

Friedberg MH, Glantz MJ, Klempner MS, Cole BF \& Perides G 1998 Specific matrix metalloproteinase profiles in the cerebrospinal fluid correlated with the presence of malignant astrocytomas, brain metastases, and carcinomatous meningitis. Cancer 82 923-930.

Friese RS, Rao F, Khandrika S, Thomas B, Ziegler MG, Schmid-Schonbein GW \& O'Connor DT 2009 Matrix metalloproteinases: discrete elevations in essential hypertension and hypertensive end-stage renal disease. Clin Exp Hypertens 31 521-533.

Gaire M, Magbanua Z, McDonnell S, McNeil L, Lovett DH \& Matrisian LM 1994 Structure and expression of the human gene for the matrix metalloproteinase matrilysin. J Biol Chem 269 2032-2040.

Gallou-Kabani C, Gabory A, Tost J, Karimi M, Mayeur S, Lesage J, Boudadi E, Gross MS, Taurelle J, Vige A, Breton C, Reusens B, Remacle C, Vieau D, Ekstrom TJ, Jais JP \& Junien C 2010 Sex- and diet-specific changes of imprinted gene expression and DNA methylation in mouse placenta under a highfat diet. PLoS One 5 e14398.

Garrido-Urbani S, Jemelin S, Deffert C, Carnesecchi S, Basset O, Szyndralewiez C, Heitz F, Page P, Montet X, Michalik L, Arbiser J, Ruegg C, Krause KH \& Imhof BA 2011 Targeting vascular NADPH oxidase 1 blocks tumor angiogenesis through a PPARalpha mediated mechanism. PLoS One 6 e14665.

Ge H, Tollner TL, Hu Z, Da M, Li X, Guan H, Shan D, Lu J, Huang C \& Dong Q 2012 Impaired mitochondrial function in murine oocytes is associated with controlled ovarian hyperstimulation and in vitro maturation. Reprod Fertil Dev 24 945-952. 
Ghulmiyyah L \& Sibai B 2012 Maternal Mortality From Preeclampsia/Eclampsia. Seminars in Perinatology 36 56-59.

Gilbert JS, Lang AL, Grant AR \& Nijland MJ 2005 Maternal nutrient restriction in sheep: hypertension and decreased nephron number in offspring at 9 months of age. J Physiol 565 137-147.

Gillman MW, Barker D, Bier D, Cagampang F, Challis J, Fall C, Godfrey K, Gluckman P, Hanson M, Kuh D, Nathanielsz P, Nestel P \& Thornburg KL 2007 Meeting report on the 3rd International Congress on Developmental Origins of Health and Disease (DOHaD). Pediatr Res 61 625-629.

Gleicher N. 2013. High FSH. Retrieved July 24, 2013 from

https://www.centerforhumanreprod.com/high_fsh.html.

Goddard MJ, \& Pratt HP 1983 Control of events during early cleavage of the mouse embryo: an analysis of the '2-cell block'. Development 73 111-133.

Gomez DE, Alonso DF, Yoshiji H \& Thorgeirsson UP 1997 Tissue inhibitors of metalloproteinases: structure, regulation and biological functions. Eur J Cell Biol 74 111-122.

Gomis-Ruth FX, Maskos K, Betz M, Bergner A, Huber R, Suzuki K, Yoshida N, Nagase H, Brew K, Bourenkov GP, Bartunik H \& Bode W 1997 Mechanism of inhibition of the human matrix metalloproteinase stromelysin-1 by TIMP-1. Nature 389 77-81.

Grazul-Bilska AT, Borowczyk E, Bilski JJ, Reynolds LP, Redmer DA, Caton JS \& Vonnahme KA 2012 Overfeeding and underfeeding have detrimental effects on oocyte quality measured by in vitro fertilization and early embryonic development in sheep. Domestic Animal Endocrinology 43 289-298.

Green JH \& Heffron PF 1968 Studies upon the relationship between baroreceptor and sympathetic activity. Q J Exp Physiol Cogn Med Sci 53 23-32.

Grodstein F, Goldman MB \& Cramer DW 1994 Body mass index and ovulatory infertility. Epidemiology 5 247-250. 
Gross J \& Lapiere CM 1962 Collagenolytic activity in amphibian tissues: a tissue culture assay. Proc Natl Acad Sci U S A 48 1014-1022.

Grote K, Flach I, Luchtefeld M, Akin E, Holland SM, Drexler H \& Schieffer B 2003 Mechanical stretch enhances mRNA expression and proenzyme release of matrix metalloproteinase-2 (MMP-2) via NAD(P)H oxidase-derived reactive oxygen species. Circ Res 92 e80-86.

Guzik B, Sagan A, Ludew D, Mrowiecki W, Chwala M, Bujak-Gizycka B, Filip G, Grudzien G, Kapelak B, Zmudka K, Mrowiecki T, Sadowski J, Korbut R \& Guzik TJ 2013 Mechanisms of oxidative stress in human aortic aneurysms Association with clinical risk factors for atherosclerosis and disease severity. Int $J$ Cardiol.

Hagler MA, Hadley TM, Zhang H, Mehra K, Roos CM, Schaff HV, Suri RM \& Miller JD 2013 TGF-beta signalling and reactive oxygen species drive fibrosis and matrix remodelling in myxomatous mitral valves. Cardiovasc Res 99 175184.

Hales CN, Barker DJ, Clark PM, Cox LJ, Fall C, Osmond C \& Winter PD 1991 Fetal and infant growth and impaired glucose tolerance at age 64. BMJ 303 10191022.

Hales CN \& Barker DJ 1992 Type 2 (non-insulin-dependent) diabetes mellitus: the thrifty phenotype hypothesis. Diabetologia 35 595-601.

Hanover LM \& White JS 1993 Manufacturing, composition, and applications of fructose. Am J Clin Nutr 58 724S-732S.

Hao L, Du M, Lopez-Campistrous A \& Fernandez-Patron C 2004 Agonist-induced activation of matrix metalloproteinase-7 promotes vasoconstriction through the epidermal growth factor-receptor pathway. Circ Res 94 68-76.

Haque MZ \& Majid DS 2011 High salt intake delayed angiotensin II-induced hypertension in mice with a genetic variant of NADPH oxidase. Am J Hypertens 24 114-118.

Harding $\mathbf{J} 2001$ The nutritional basis of the fetal origins of adult disease. Int J Epidemiol 30 15-23. 
Harper AM, Chaplin MF \& Segal AW 1985 Cytochrome b-245 from human neutrophils is a glycoprotein. Biochem $J$ 227 783-788.

\section{Hashida S, Yuzawa S, Suzuki NN, Fujioka Y, Takikawa T, Sumimoto H, Inagaki F} \& Fujii H 2004 Binding of FAD to cytochrome b558 is facilitated during activation of the phagocyte NADPH oxidase, leading to superoxide production. $J$ Biol Chem 279 26378-26386.

Hayes E, Lechowicz A, Petrik J, Storozhuk Y, Paez-Parent S, Dai Q, Samjoo I, Mansell M, Gruslin A, Holloway A \& Raha S 2012 Adverse fetal and neonatal outcomes associated with a life-long high fat diet: role of altered development of the placental vasculature. PLoS One 7 e33370.

Heagerty AM, Aalkjaer C, Bund SJ, Korsgaard N \& Mulvany MJ 1993 Small artery structure in hypertension. Dual processes of remodeling and growth. Hypertension 21 391-397.

Hendry C, Farley A \& McLafferty E 2012 Blood vessels, circulation and blood pressure. Nurs Stand 27 35-40.

Henriksen T 2008 The macrosomic fetus: a challenge in current obstetrics. Acta Obstet Gynecol Scand 87 134-145.

Henry JB, Miller MC, Kelly KC \& Champney D 2002 Mean arterial pressure (MAP): an alternative and preferable measurement to systolic blood pressure (SBP) in patients for hypotension detection during hemapheresis. J Clin Apher 17 55-64.

Herrera-Acosta J, Perez-Grovas H, Fernandez M \& Arriaga J 1985 Enalapril in essential hypertension. Drugs 30 Suppl 1 35-46.

Hollander W 1976 Role of hypertension in atherosclerosis and cardiovascular disease. Am J Cardiol 38 786-800.

Hollander W, Kramsch DM, Farmelant M \& Madoff IM 1968 Arterial wall metabolism in experimental hypertension of coarctation of the aorta of short duration. J Clin Invest 47 1221-1229. 
Hollander W, Prusty S, Kemper T, Rosene DL \& Moss MB 1993 The effects of hypertension on cerebral atherosclerosis in the cynomolgus monkey. Stroke $\mathbf{2 4}$ 1218-1226; discussion 1226-1217.

Horsthemke B \& Ludwig M 2005 Assisted reproduction: the epigenetic perspective. Hum Reprod Update 11 473-482.

Hwang IS, Ho H, Hoffman BB \& Reaven GM 1987 Fructose-induced insulin resistance and hypertension in rats. Hypertension 10 512-516.

IDF. 2013. Impaired Glucose Tolerance (IGT). Retrieved July 24, 201 from http://www.idf.org/diabetesatlas/5e/impaired-glucose-tolerance.

Igosheva N, Abramov AY, Poston L, Eckert JJ, Fleming TP, Duchen MR \& McConnell J 2010 Maternal diet-induced obesity alters mitochondrial activity and redox status in mouse oocytes and zygotes. PLoS One 5 e10074.

Iguchi A, Yamakage H, Tochiya M, Muranaka K, Sasaki Y, Kono S, Shimatsu A \& Satoh-Asahara N 2013 Effects of weight reduction therapy on obstructive sleep apnea syndrome and arterial stiffness in patients with obesity and metabolic syndrome. J Atheroscler Thromb. Article ID 17632, 14 pages, 2013, doi: http://dx.doi.org/10.5551/jat.17632.

Isensee J, Witt H, Pregla R, Hetzer R, Regitz-Zagrosek V \& Noppinger PR 2008 Sexually dimorphic gene expression in the heart of mice and men. $J$ Mol Med (Berl) 86 61-74.

Ishimoto T, Lanaspa MA, Rivard CJ, Roncal-Jimenez CA, Orlicky DJ, Cicerchi C, McMahan RH, Abdelmalek MF, Rosen HR, Jackman MR, Maclean PS, Diggle CP, Asipu A, Inaba S, Kosugi T, Sato W, Maruyama S, SanchezLozada LG, Sautin YY, Hill JO, Bonthron DT \& Johnson RJ 2013 High fat and high sucrose (western) diet induce steatohepatitis that is dependent on fructokinase. Hepatology. Article ID 23813872, 12 pages, 2013, doi: 10.1002/hep.26594.

Itoh Y \& Nagase H 1995 Preferential inactivation of tissue inhibitor of metalloproteinases-1 that is bound to the precursor of matrix metalloproteinase 9 (progelatinase B) by human neutrophil elastase. J Biol Chem 270 16518-16521. 
Jen KL, Rochon C, Zhong SB \& Whitcomb L 1991 Fructose and sucrose feeding during pregnancy and lactation in rats changes maternal and pup fuel metabolism. J Nutr 121 1999-2005.

Jensen TK, Scheike T, Keiding N, Schaumburg I \& Grandjean P 1999 Fecundability in relation to body mass and menstrual cycle patterns. Epidemiology 10 422-428.

Ji Y, Liu J, Wang Z \& Liu N 2009 Angiotensin II induces inflammatory response partly via toll-like receptor 4-dependent signaling pathway in vascular smooth muscle cells. Cell Physiol Biochem 23 265-276.

Jiao GZ, Cao XY, Cui W, Lian HY, Miao YL, Wu XF, Han D \& Tan JH 2013 Developmental potential of prepubertal mouse oocytes is compromised due mainly to their impaired synthesis of glutathione. PLoS One 8 e58018.

Jousilahti P, Vartiainen E, Tuomilehto J \& Puska P 1999 Sex, age, cardiovascular risk factors, and coronary heart disease: a prospective follow-up study of 14786 middle-aged men and women in Finland. Circulation 99 1165-1172.

\section{Jungheim ES, Louden ED, Chi MM, Frolova AI, Riley JK \& Moley KH 2011} Preimplantation exposure of mouse embryos to palmitic acid results in fetal growth restriction followed by catch-up growth in the offspring. Biol Reprod $\mathbf{8 5}$ 678-683.

Kajantie E, Barker DJ, Osmond C, Forsen T \& Eriksson JG 2008 Growth before 2 years of age and serum lipids 60 years later: the Helsinki Birth Cohort study. Int $J$ Epidemiol 37 280-289.

Kajantie E, Osmond C, Barker DJ, Forsen T, Phillips DI \& Eriksson JG 2005 Size at birth as a predictor of mortality in adulthood: a follow-up of 350000 personyears. Int J Epidemiol 34 655-663.

Kallen B, Finnstrom O, Lindam A, Nilsson E, Nygren KG \& Otterblad PO 2010 Congenital malformations in infants born after in vitro fertilization in Sweden. Birth Defects Res A Clin Mol Teratol 88 137-143.

Kalra SK, Ratcliffe SJ, Coutifaris C, Molinaro T \& Barnhart KT 2011 Ovarian stimulation and low birth weight in newborns conceived through in vitro fertilization. Obstet Gynecol 118 863-871. 
Kaplan NL, E. 2002 Kaplan's Clinical Hypertension. Baltimore, MD, USA: Lippincott Williams \& Wilkins.

Katakam PV, Tulbert CD, Snipes JA, Erdos B, Miller AW \& Busija DW 2005 Impaired insulin-induced vasodilation in small coronary arteries of Zucker obese rats is mediated by reactive oxygen species. Am J Physiol Heart Circ Physiol 288 H854-860.

Katalinic A, Rösch C \& Ludwig M 2004 Pregnancy course and outcome after intracytoplasmic sperm injection: a controlled, prospective cohort study. Fertil Steril 81 1604-1616.

Katsikis I, Kita M, Karkanaki A, Prapas N \& Panidis D 2006 Anovulation and ovulation induction. Hippokratia 10 120-127.

Keeling WB, Armstrong PA, Stone PA, Bandyk DF \& Shames ML 2005 An overview of matrix metalloproteinases in the pathogenesis and treatment of abdominal aortic aneurysms. Vasc Endovascular Surg 39 457-464.

Kelly T, Yang W, Chen CS, Reynolds K \& He J 2008 Global burden of obesity in 2005 and projections to 2030. Int J Obes 32 1431-1437.

Kelsall CJ, Hoile SP, Irvine NA, Masoodi M, Torrens C, Lillycrop KA, Calder PC, Clough GF, Hanson MA \& Burdge GC 2012 Vascular dysfunction induced in offspring by maternal dietary fat involves altered arterial polyunsaturated fatty acid biosynthesis. PLoS One 7 e34492.

Khan JA, Abdul Rahman MN, Mazari FA, Shahin Y, Smith G, Madden L, Fagan MJ, Greenman J, McCollum PT \& Chetter IC 2012 Intraluminal thrombus has a selective influence on matrix metalloproteinases and their inhibitors (tissue inhibitors of matrix metalloproteinases) in the wall of abdominal aortic aneurysms. Ann Vasc Surg 26 322-329.

Klebanoff SJ 1980 Oxygen metabolism and the toxic properties of phagocytes. Ann Intern Med 93 480-489.

Korsgaard N, Aalkjaer C, Heagerty AM, Izzard AS \& Mulvany MJ 1993 Histology of subcutaneous small arteries from patients with essential hypertension. Hypertension 22 523-526. 
Lake JK, Power C \& Cole TJ 1997 Women's reproductive health: the role of body mass index in early and adult life. Int J Obes Relat Metab Disord 21 432-438.

Lan X, Cretney EC, Kropp J, Khateeb K, Berg MA, Penagaricano F, Magness R, Radunz AE \& Khatib H 2013 Maternal Diet during Pregnancy Induces Gene Expression and DNA Methylation Changes in Fetal Tissues in Sheep. Front Genet 449.

Lavinsky L, Oliveira MW, Bassanesi HJ, D'Avila C \& Lavinsky M 2004 Hyperinsulinemia and tinnitus: a historical cohort. Int Tinnitus J 10 24-30.

Lawlor DA, Najman JM, Sterne J, Williams GM, Ebrahim S \& Davey Smith G 2004 Associations of parental, birth, and early life characteristics with systolic blood pressure at 5 years of age: findings from the Mater-University study of pregnancy and its outcomes. Circulation 110 2417-2423.

Lawlor DA, Smith GD, O'Callaghan M, Alati R, Mamun AA, Williams GM \& Najman JM 2007 Epidemiologic evidence for the fetal overnutrition hypothesis: findings from the mater-university study of pregnancy and its outcomes. Am J Epidemiol 165 418-424.

Leco KJ, Harvey MB, Hogan A, Copeland NG, Gilbert DJ, Jenkins NA, Edwards DR \& Schultz GA 1997 Matrix metalloproteinase-9 maps to the distal end of chromosome 2 in the mouse. Dev Genet 21 55-60.

Lehoux S, Lemarie CA, Esposito B, Lijnen HR \& Tedgui A 2004 Pressure-induced matrix metalloproteinase-9 contributes to early hypertensive remodeling. Circulation 109 1041-1047.

Li WG, Miller FJ, Jr., Zhang HJ, Spitz DR, Oberley LW \& Weintraub NL 2001 $\mathrm{H}(2) \mathrm{O}(2)$-induced $\mathrm{O}(2)$ production by a non-phagocytic NAD(P)H oxidase causes oxidant injury. J Biol Chem 276 29251-29256.

Liang C, DeCourcy K \& Prater MR 2010 High-saturated-fat diet induces gestational diabetes and placental vasculopathy in C57BL/6 mice. Metabolism 59 943-950.

Liew G, Mitchell P, Rochtchina E, Wong TY, Hsu W, Lee ML, Wainwright A \& Wang JJ 2011 Fractal analysis of retinal microvasculature and coronary heart disease mortality. Eur Heart J 32 422-429. 
Lindsey ML \& Zamilpa R 2012 Temporal and spatial expression of matrix metalloproteinases and tissue inhibitors of metalloproteinases following myocardial infarction. Cardiovasc Ther 30 31-41.

Lintsen AM, Pasker-de Jong PC, de Boer EJ, Burger CW, Jansen CA, Braat DD \& van Leeuwen FE 2005 Effects of subfertility cause, smoking and body weight on the success rate of IVF. Hum Reprod 20 1867-1875.

London GM \& Pannier B 2010 Arterial functions: how to interpret the complex physiology. Nephrol Dial Transplant 25 3815-3823.

Longo GM, Xiong W, Greiner TC, Zhao Y, Fiotti N \& Baxter BT 2002 Matrix metalloproteinases 2 and 9 work in concert to produce aortic aneurysms. J Clin Invest 110 625-632.

Luke B, Brown MB, Missmer SA, Bukulmez O, Leach R \& Stern JE 2011a The effect of increasing obesity on the response to and outcome of assisted reproductive technology: a national study. Fertil Steril 96 820-825.

Luke B, Brown MB, Stern JE, Missmer SA, Fujimoto VY \& Leach R 2011b Female obesity adversely affects assisted reproductive technology (ART) pregnancy and live birth rates. Hum Reprod 26 245-252.

Luo C, Zuniga J, Edison E, Palla S, Dong W \& Parker-Thornburg J 2011 Superovulation strategies for 6 commonly used mouse strains. J Am Assoc Lab Anim Sci 50 471-478.

Lussana F, Painter RC, Ocke MC, Buller HR, Bossuyt PM \& Roseboom TJ 2008 Prenatal exposure to the Dutch famine is associated with a preference for fatty foods and a more atherogenic lipid profile. Am J Clin Nutr 88 1648-1652.

Luzzo KM, Wang Q, Purcell SH, Chi M, Jimenez PT, Grindler N, Schedl T \& Moley KH 2012 High fat diet induced developmental defects in the mouse: oocyte meiotic aneuploidy and fetal growth retardation/brain defects. PLoS One 7 e49217.

\section{Machtinger R, Combelles CM, Missmer SA, Correia KF, Fox JH \& Racowsky C} 2012 The association between severe obesity and characteristics of failed fertilized oocytes. Hum Reprod 27 3198-3207. 
MacLeod J 1943 The Role of Oxygen in the Metabolism and Motility of Human Spermatozoa. Am J Physiol 138 512-518.

Magliano DC, Bargut TC, de Carvalho SN, Aguila MB, Mandarim-de-Lacerda CA \& Souza-Mello V 2013 Peroxisome proliferator-activated receptors-alpha and gamma are targets to treat offspring from maternal diet-induced obesity in mice. PLoS One 8 e64258.

Mahajan N, Dhawan V, Mahmood S, Malik S \& Jain S 2012 Extracellular matrix remodeling in Takayasu's arteritis: role of matrix metalloproteinases and adventitial inflammation. Arch Med Res 43 406-410.

Maher E, Afnan M, \& Barratt, CL. 2003 Epigenetic risks related to assisted reproductive technologies: Epigenetics, imprinting, ART and icebergs? Human Reproduction 18 2508-2511.

Maheshwari A, Stofberg L \& Bhattacharya S 2007 Effect of overweight and obesity on assisted reproductive technology--a systematic review. Hum Reprod Update 13 433-444.

Maloney CA, Hay SM, Young LE, Sinclair KD \& Rees WD 2011 A methyl-deficient diet fed to rat dams during the peri-conception period programs glucose homeostasis in adult male but not female offspring. J Nutr 141 95-100.

Maltepe E, Bakardjiev AI \& Fisher SJ 2010 The placenta: transcriptional, epigenetic, and physiological integration during development. J Clin Invest 120 1016-1025.

\section{Mamun AA, O'Callaghan M, Callaway L, Williams G, Najman J \& Lawlor DA} 2009 Associations of gestational weight gain with offspring body mass index and blood pressure at 21 years of age: evidence from a birth cohort study. Circulation $1191720-1727$.

Marriott BP, Cole N \& Lee E 2009 National estimates of dietary fructose intake increased from 1977 to 2004 in the United States. J Nutr 139 1228S-1235S.

Martinez-Lemus LA \& Galinanes EL 2011 Matrix metalloproteinases and small artery remodeling. Drug Discov Today Dis Models 8 21-28. 
Martinez-Lemus LA, Zhao G, Galinanes EL \& Boone M 2011 Inward remodeling of resistance arteries requires reactive oxygen species-dependent activation of matrix metalloproteinases. Am J Physiol Heart Circ Physiol 300 H2005-2015.

Mathai ML, Soueid M, Chen N, Jayasooriya AP, Sinclair AJ, Wlodek ME, Weisinger HS \& Weisinger RS 2004 Does perinatal omega-3 polyunsaturated fatty acid deficiency increase appetite signaling? Obes Res 12 1886-1894.

Mathiassen ON, Buus NH, Sihm I, Thybo NK, Morn B, Schroeder AP, Thygesen K, Aalkjaer C, Lederballe O, Mulvany MJ \& Christensen KL 2007 Small artery structure is an independent predictor of cardiovascular events in essential hypertension. J Hypertens 25 1021-1026.

Mayer J. 2013. Week 2: systemic circuit \& peripheral circulation. Retrieved July 23, 2013from http://www.studyblue.com/notes/note/n/week-2-systematic-circuit-peripheral-circulation/deck/6260394.

Metwally M, Cutting R, Tipton A, Skull J, Ledger WL \& Li TC 2007 Effect of increased body mass index on oocyte and embryo quality in IVF patients. Reprod Biomed Online 15 532-538.

Minge CE, Bennett BD, Norman RJ \& Robker RL 2008 Peroxisome proliferatoractivated receptor-gamma agonist rosiglitazone reverses the adverse effects of diet-induced obesity on oocyte quality. Endocrinology 149 2646-2656.

Mistry HD, Kurlak LO \& Broughton Pipkin F 2013 The placental renin-angiotensin system and oxidative stress in pre-eclampsia. Placenta 34 182-186.

Montezano AC \& Touyz RM 2013 Reactive oxygen species, vascular Noxs, and hypertension: Focus on translational and clinical research. Antioxid Redox Signal. Article ID 23600794, 19 pages, 2013, doi: 10.1089/ars.2013.5032.

Morandi A, Meyre D, Lobbens S, Kleinman K, Kaakinen M, Rifas-Shiman SL, Vatin V, Gaget S, Pouta A, Hartikainen AL, Laitinen J, Ruokonen A, Das S, Khan AA, Elliott P, Maffeis C, Gillman MW, Jarvelin MR \& Froguel P 2012 Estimation of newborn risk for child or adolescent obesity: lessons from longitudinal birth cohorts. PLoS One 7 e49919.

Muhlhausler BS, Duffield JA \& McMillen IC 2007 Increased maternal nutrition stimulates peroxisome proliferator activated receptor-gamma, adiponectin, and 
leptin messenger ribonucleic acid expression in adipose tissue before birth. Endocrinology 148 878-885.

Mulvany MJ 1999 Vascular remodelling of resistance vessels: can we define this? Cardiovasc Res 41 9-13.

Mulvany MJ \& Aalkjaer C 1990 Structure and function of small arteries. Physiol Rev 70 921-961.

Murphy G, Houbrechts A, Cockett MI, Williamson RA, O'Shea M \& Docherty AJ 1991 The $\mathrm{N}$-terminal domain of tissue inhibitor of metalloproteinases retains metalloproteinase inhibitory activity. Biochemistry 30 8097-8102.

Nagae G, Isagawa T, Shiraki N, Fujita T, Yamamoto S, Tsutsumi S, Nonaka A, Yoshiba S, Matsusaka K, Midorikawa Y, Ishikawa S, Soejima H, Fukayama M, Suemori H, Nakatsuji N, Kume S \& Aburatani H 2011 Tissue-specific demethylation in CpG-poor promoters during cellular differentiation. Hum Mol Genet 20 2710-2721.

Nagase H 1997 Activation mechanisms of matrix metalloproteinases. Biol Chem $\mathbf{3 7 8}$ 151-160.

Nagase H \& Woessner JF, Jr. 1999 Matrix metalloproteinases. J Biol Chem 274 2149121494.

Nagy A GM, Vintersten K, Behringer R. 2003 Manipulating the Mouse Embryo, A laboratory manual, third edition.: Cold Spring Harbor Laboratory Press.

NCI. 2012. SEER Training Modules: Introduction to the Cardiovascular System July 20, 2013.

Negron-Perez VM, Echevarria FD, Huffman SR \& Rivera RM 2013 Determination of allelic expression of h19 in pre- and peri-implantation mouse embryos. Biol Reprod 8897.

Nelissen EC, Van Montfoort AP, Coonen E, Derhaag JG, Geraedts JP, Smits LJ, Land JA, Evers JL \& Dumoulin JC 2012 Further evidence that culture media affect perinatal outcome: findings after transfer of fresh and cryopreserved embryos. Hum Reprod 27 1966-1976. 
Nelson SM, Matthews P \& Poston L 2010 Maternal metabolism and obesity: modifiable determinants of pregnancy outcome. Hum Reprod Update 16 255-275.

NIH 2012 Overweight and obesity statistics. Retrieved July 23, 2013 from http://win.niddk.nih.gov/publications/PDFs/stat904z.pdf.

O'Brien TE, Ray JG \& Chan WS 2003 Maternal body mass index and the risk of preeclampsia: a systematic overview. Epidemiology 14 368-374.

Odenbach J, Wang X, Cooper S, Chow FL, Oka T, Lopaschuk G, Kassiri Z \& Fernandez-Patron C 2011 MMP-2 mediates angiotensin II-induced hypertension under the transcriptional control of MMP-7 and TACE. Hypertension 57 123-130.

Okamoto T, Akaike T, Sawa T, Miyamoto Y, van der Vliet A \& Maeda H 2001 Activation of matrix metalloproteinases by peroxynitrite-induced protein Sglutathiolation via disulfide S-oxide formation. J Biol Chem 276 29596-29602.

Olson CK, Keppler-Noreuil KM, Romitti PA, Budelier WT, Ryan G, Sparks AET \& Van Voorhis BJ 2005 In vitro fertilization is associated with an increase in major birth defects. Fertil Steril 84 1308-1315.

Onogi A, Naruse K, Sado T, Tsunemi T, Shigetomi H, Noguchi T, Yamada Y, Akasaki M, Oi H \& Kobayashi H 2011 Hypoxia inhibits invasion of extravillous trophoblast cells through reduction of matrix metalloproteinase (MMP)-2 activation in the early first trimester of human pregnancy. Placenta 32 $665-670$.

Osmond C, Kajantie E, Forsen TJ, Eriksson JG \& Barker DJ 2007 Infant growth and stroke in adult life: the Helsinki birth cohort study. Stroke 38 264-270.

Osmond C, Barker DJ, Winter PD, Fall CH, \& Simmonds SJ. 1993 Early growth and death from cardiovascular disease in women. BMJ 307 1519-1524.

Painter RC, de Rooij SR, Bossuyt PM, de Groot E, Stok WJ, Osmond C, Barker DJ, Bleker OP \& Roseboom TJ 2007 Maternal nutrition during gestation and carotid arterial compliance in the adult offspring: the Dutch famine birth cohort. $J$ Hypertens 25 533-540. 
Painter RC, de Rooij SR, Bossuyt PM, Phillips DI, Osmond C, Barker DJ, Bleker OP \& Roseboom TJ 2006a Blood pressure response to psychological stressors in adults after prenatal exposure to the Dutch famine. J Hypertens 24 1771-1778.

Painter RC, de Rooij SR, Bossuyt PM, Simmers TA, Osmond C, Barker DJ, Bleker OP \& Roseboom TJ 2006b Early onset of coronary artery disease after prenatal exposure to the Dutch famine. Am J Clin Nutr 84 322-327.

Painter RC, De Rooij SR, Bossuyt PMM, Osmond C, Barker DJP, Bleker OP \& Roseboom TJ 2006c A possible link between prenatal exposure to famine and breast cancer: A preliminary study. American Journal of Human Biology 18 853856.

Painter RC, Osmond C, Gluckman P, Hanson M, Phillips DI \& Roseboom TJ 2008 Transgenerational effects of prenatal exposure to the Dutch famine on neonatal adiposity and health in later life. BJOG 115 1243-1249.

Panchal SK, Poudyal H, Iyer A, Nazer R, Alam MA, Diwan V, Kauter K, Sernia C, Campbell F, Ward L, Gobe G, Fenning A \& Brown L 2011 Highcarbohydrate, high-fat diet-induced metabolic syndrome and cardiovascular remodeling in rats. J Cardiovasc Pharmacol 57 611-624.

Paul M, Poyan Mehr A \& Kreutz R 2006 Physiology of local renin-angiotensin systems. Physiol Rev 86 747-803.

Paulson RJ, Sauer MV, \& Lobo RA 1990 Embryo implantation after human in vitro fertilization: importance of endometrial receptivity. Fertil Steril 53 870-874.

Pfeifer N, Baston-Bust DM, Hirchenhain J, Friebe-Hoffmann U, Rein DT, Krussel JS \& Hess AP 2012 Selection of the In Vitro Culture Media Influences mRNA Expression of Hedgehog Genes, Il-6, and Important Genes regarding Reactive Oxygen Species in Single Murine Preimplantation Embryos. ScientificWorldJournal 2012479315.

Pistea A, Bakker EN, Spaan JA \& VanBavel E 2005 Flow inhibits inward remodeling in cannulated porcine small coronary arteries. Am J Physiol Heart Circ Physiol 289 H2632-2640. 
Pollock NK, Bundy V, Kanto W, Davis CL, Bernard PJ, Zhu H, Gutin B \& Dong Y 2012 Greater fructose consumption is associated with cardiometabolic risk markers and visceral adiposity in adolescents. J Nutr 142 251-257.

Poston L 2011 Influence of maternal nutritional status on vascular function in the offspring. Microcirculation 18 256-262.

Prewitt RL, Rice DC \& Dobrian AD 2002 Adaptation of resistance arteries to increases in pressure. Microcirculation 9 295-304.

Radavelli-Bagatini S, Blair AR, Proietto J, Spritzer PM \& Andrikopoulos S 2011 The New Zealand obese mouse model of obesity insulin resistance and poor breeding performance: evaluation of ovarian structure and function. $J$ Endocrinol 209 307-315.

Raffetto JD \& Khalil RA 2008 Matrix metalloproteinases and their inhibitors in vascular remodeling and vascular disease. Biochem Pharmacol 75 346-359.

Rajagopalan S, Meng XP, Ramasamy S, Harrison DG \& Galis ZS 1996 Reactive oxygen species produced by macrophage-derived foam cells regulate the activity of vascular matrix metalloproteinases in vitro. Implications for atherosclerotic plaque stability. J Clin Invest 98 2572-2579.

Rasmussen SA, Chu SY, Kim SY, Schmid CH \& Lau J 2008 Maternal obesity and risk of neural tube defects: a metaanalysis. Am J Obstet Gynecol 198 611-619.

Ravelli AC, van Der Meulen JH, Osmond C, Barker DJ \& Bleker OP 1999 Obesity at the age of $50 \mathrm{y}$ in men and women exposed to famine prenatally. Am J Clin Nutr 70 811-816.

Ravelli ACJ, Van der Meulen JHP, Michels RPJ, Osmond C, Barker DJP, Hales CN \& Bleker OP 1998 Glucose tolerance in adults after prenatal exposure to famine. Lancet 351173.

Rawana S, Clark K, Zhong S, Buison A, Chackunkal S \& Jen KL 1993 Low dose fructose ingestion during gestation and lactation affects carbohydrate metabolism in rat dams and their offspring. J Nutr 123 2158-2165.

Razin A \& Riggs AD 1980 DNA methylation and gene function. Science 210 604-610. 
Red-Horse K, Zhou Y, Genbacev O, Prakobphol A, Foulk R, McMaster M \& Fisher SJ 2004 Trophoblast differentiation during embryo implantation and formation of the maternal-fetal interface. J Clin Invest 114 744-754.

Reefhuis J, Honein MA, Schieve LA, Correa A, Hobbs CA \& Rasmussen SA 2009 Assisted reproductive technology and major structural birth defects in the United States. Hum Reprod 24 360-366.

Reminetskyy BY. Vessels and nerves of thorax. Anterior branches of thoracic spinal nerves. Retrieved July 23, 2013 from http://intranet.tdmu.edu.ua/data/ kafedra/internal/anatomy/classes_stud/en/stomat/ptn/1/28_\%20VESSELS\%20AN D\%20NERVES\%20OF\%20THORAX.\%20ANTERIOR\%20BRANCHES\%20OF \%20THORACIC\%20SPINAL\%20NERVES.files/image012.gif..

Ren J 2004 Leptin and hyperleptinemia - from friend to foe for cardiovascular function. J Endocrinol 181 1-10.

Rich-Edwards JW, Goldman MB, Willett WC, Hunter DJ, Stampfer MJ, Colditz GA \& Manson JE 1994 Adolescent body mass index and infertility caused by ovulatory disorder. Am J Obstet Gynecol 171 171-177.

Rinaudo P \& Schultz RM 2004 Effects of embryo culture on global pattern of gene expression in preimplantation mouse embryos. Reproduction 128 301-311.

Rizzoni D, Palombo C, Porteri E, Muiesan ML, Kozakova M, La Canna G, Nardi M, Guelfi D, Salvetti M, Morizzo C, Vittone F \& Rosei EA 2003a Relationships between coronary flow vasodilator capacity and small artery remodelling in hypertensive patients. J Hypertens 21 625-631.

Rizzoni D, Porteri E, Boari GE, De Ciuceis C, Sleiman I, Muiesan ML, Castellano M, Miclini M \& Agabiti-Rosei E 2003b Prognostic significance of small-artery structure in hypertension. Circulation 108 2230-2235.

Rizzoni D, Porteri E, Castellano M, Bettoni G, Muiesan ML, Muiesan P, Giulini SM \& Agabiti-Rosei E 1996 Vascular hypertrophy and remodeling in secondary hypertension. Hypertension 28 785-790.

Robertson WB \& Strong JP 1968 Atherosclerosis in persons with hypertension and diabetes mellitus. Lab Invest 18 538-551. 
Robinson SA, Batelaan SF, Syddall HE, Sayer AA, Dennison EM, Martin HJ, Barker DJ, Cooper C, \& Hertfordshire Cohort Study Group 2006 Combined effects of dietary fat and birth weight on serum cholesterol concentrations: the Hertfordshire Cohort Study. Am J Clin Nutr 84 237-244.

Robker RL 2008 Evidence that obesity alters the quality of oocytes and embryos. Pathophysiology 15 115-121.

Robker RL, Akison LK, Bennett BD, Thrupp PN, Chura LR, Russell DL, Lane M \& Norman RJ 2009 Obese women exhibit differences in ovarian metabolites, hormones, and gene expression compared with moderate-weight women. J Clin Endocrinol Metab 94 1533-1540.

Rodrigues SF, Tran ED, Fortes ZB \& Schmid-Schonbein GW 2010 Matrix metalloproteinases cleave the beta2-adrenergic receptor in spontaneously hypertensive rats. Am J Physiol Heart Circ Physiol 299 H25-35.

Roseboom T, de Rooij S \& Painter R 2006 The Dutch famine and its long-term consequences for adult health. Early Hum Dev 82 485-491.

Roseboom TvdM, JHP; van Montfrans, GA; Ravelli, ACJ; Osmond, C; Barker, DJP; Bleker, OP. 2001 Maternal nutrition during gestation and blood pressure in later life. Hypertension 19 29-34.

Ross R \& Glomset JA 1976 The pathogenesis of atherosclerosis (first of two parts). $N$ Engl J Med 295 369-377.

Rossi F \& Zatti M 1964 Biochemical aspects of phagocytosis in polymorphonuclear leucocytes. NADH and NADPH oxidation by the granules of resting and phagocytizing cells. Experientia 20 21-23.

Royer-Pokora B, Kunkel LM, Monaco AP, Goff SC, Newburger PE, Baehner RL, Cole FS, Curnutte JT \& Orkin SH 1986 Cloning the gene for an inherited human disorder--chronic granulomatous disease--on the basis of its chromosomal location. Nature 322 32-38.

Sakka SD, Loutradis D, Kanaka-Gantenbein C, Margeli A, Papastamataki M, Papassotiriou I \& Chrousos GP 2010 Absence of insulin resistance and lowgrade inflammation despite early metabolic syndrome manifestations in children born after in vitro fertilization. Fertil Steril 94 1693-1699. 
Sakurada M, Yoshimoto T, Sekizawa N, Hirono Y, Suzuki N \& Hirata Y 2010 Vasculoprotective effect of cilostazol in aldosterone-induced hypertensive rats. Hypertens Res 33 229-235.

Salo T, Makela M, Kylmaniemi M, Autio-Harmainen H \& Larjava H 1994 Expression of matrix metalloproteinase-2 and -9 during early human wound healing. Lab Invest 70 176-182.

Salonen MK, Kajantie E, Osmond C, Forsén T, Ylihärsilä H, Paile-Hyvärinen M, Barker DJP \& Eriksson JG 2011 Developmental Origins of Physical Fitness: The Helsinki Birth Cohort Study. PLoS One 6 e22302.

Saltin B, Radegran G, Koskolou MD \& Roach RC 1998 Skeletal muscle blood flow in humans and its regulation during exercise. Acta Physiol Scand 162 421-436.

Samuelsson AM, Matthews PA, Argenton M, Christie MR, McConnell JM, Jansen EH, Piersma AH, Ozanne SE, Twinn DF, Remacle C, Rowlerson A, Poston L \& Taylor PD 2008 Diet-induced obesity in female mice leads to offspring hyperphagia, adiposity, hypertension, and insulin resistance: a novel murine model of developmental programming. Hypertension 51 383-392.

Samuelsson AM, Morris A, Igosheva N, Kirk SL, Pombo JM, Coen CW, Poston L \& Taylor PD 2010 Evidence for sympathetic origins of hypertension in juvenile offspring of obese rats. Hypertension 55 76-82.

SART. 2013. Clinical Summary Report. Retrieved July 24, 2013fom https: //www.sartcorsonline.com/rptCSR_PublicMultYear.aspx?ClinicPKID=0.

Sauber-Schatz EK, Sappenfield W, Grigorescu V, Kulkarni A, Zhang Y, Salihu HM, Rubin LP, Kirby RS, Jamieson DJ \& Macaluso M 2012 Obesity, assisted reproductive technology, and early preterm birth--Florida, 2004-2006. Am J Epidemiol 176 886-896.

Schachter M, Raziel A, Friedler S, Strssburger D, Bern O, \& Ron-El R 2001 Monozygotic twinning after assisted reproductive techniques: a phenomenon independent of micromanipulation. Human Reproduction 16 1264-1269.

Scherrer U, Rimoldi SF, Rexhaj E, Stuber T, Duplain H, Garcin S, de Marchi SF, Nicod P, Germond M, Allemann Y \& Sartori C 2012 Systemic and pulmonary 
vascular dysfunction in children conceived by assisted reproductive technologies. Circulation 125 1890-1896.

\section{Schwartzkopff B, Motz W, Frenzel H, Vogt M, Knauer S \& Strauer BE 1993}

Structural and functional alterations of the intramyocardial coronary arterioles in patients with arterial hypertension. Circulation 88 993-1003.

Scott KA, Yamazaki Y, Yamamoto M, Lin Y, Melhorn SJ, Krause EG, Woods SC, Yanagimachi R, Sakai RR \& Tamashiro KL 2010 Glucose parameters are altered in mouse offspring produced by assisted reproductive technologies and somatic cell nuclear transfer. Biol Reprod 83 220-227.

\section{Sebire NJ, Jolly M, Harris JP, Wadsworth J, Joffe M, Beard RW, Regan L \&} Robinson S 2001 Maternal obesity and pregnancy outcome: a study of 287,213 pregnancies in London. Int J Obes Relat Metab Disord 25 1175-1182.

Sellers A, Reynolds JJ \& Meikle MC 1978 Neutral metallo-proteinases of rabbit bone. Separation in latent forms of distinct enzymes that when activated degrade collagen, gelatin and proteoglycans. Biochem J 171 493-496.

Sellers A \& Woessner JF, Jr. 1980 The extraction of a neutral metalloproteinase from the involuting rat uterus, and its action on cartilage proteoglycan. Biochem J 189 521-531.

Sesso R \& Franco MC 2010 Abnormalities in metalloproteinase pathways and IGF-I axis: a link between birth weight, hypertension, and vascular damage in childhood. Am J Hypertens 23 6-11.

Shah DK, Missmer SA, Berry KF, Racowsky C \& Ginsburg ES 2011 Effect of obesity on oocyte and embryo quality in women undergoing in vitro fertilization. Obstet Gynecol 118 63-70.

Shankar K, Kang P, Harrell A, Zhong Y, Marecki JC, Ronis MJ \& Badger TM 2010 Maternal overweight programs insulin and adiponectin signaling in the offspring. Endocrinology 151 2577-2589.

Shankar K, Zhong Y, Kang P, Lau F, Blackburn ML, Chen JR, Borengasser SJ, Ronis MJ \& Badger TM 2011 Maternal obesity promotes a proinflammatory signature in rat uterus and blastocyst. Endocrinology 152 4158-4170. 
Shertzer HG, Krishan M \& Genter MB 2013 Dietary whey protein stimulates mitochondrial activity and decreases oxidative stress in mouse female brain. Neurosci Lett 548 159-164.

Shimada I, Matsui K, Iida R, Tsubota E \& Matsuki T 2009 Time course of housekeeping gene expression changes in diffuse alveolar damage induced by hyperoxia exposure in mice. Leg Med (Tokyo) 11 Suppl 1 S151-154.

Simoes-Silva L, Moreira-Rodrigues M, Quelhas-Santos J, Fernandes-Cerqueira C, Pestana M, Soares-Silva I \& Sampaio-Maia B 2013 Intestinal and renal guanylin peptides system in hypertensive obese mice. Exp Biol Med (Maywood) 238 90-97.

Siwik DA, Pagano PJ \& Colucci WS 2001 Oxidative stress regulates collagen synthesis and matrix metalloproteinase activity in cardiac fibroblasts. Am J Physiol Cell Physiol 280 C53-60.

\section{Skalnik DG, Dorfman DM, Perkins AS, Jenkins NA, Copeland NG \& Orkin SH} 1991 Targeting of transgene expression to monocyte/macrophages by the gp91phox promoter and consequent histiocytic malignancies. Proc Natl Acad Sci U S A 88 8505-8509.

Smith CA 1947 The effect of wartime starvation in Holland upon pregnancy and its product. Am J Obstet Gynecol 53 599-608.

Sorensen HT, Sabroe S, Rothman KJ, Gillman M, Fischer P \& Sorensen TI 1997 Relation between weight and length at birth and body mass index in young adulthood: cohort study. BMJ 3151137.

Spandorfer SD, Kump L, Goldschlag D, Brodkin T, Davis OK \& Rosenwaks Z 2004 Obesity and in vitro fertilization: negative influences on outcome. $J$ Reprod Med 49 973-977.

Sukumar P, Viswambharan H, Imrie H, Cubbon RM, Yuldasheva N, Gage M, Galloway S, Skromna A, Kandavelu P, Santos CX, Gatenby VK, Smith J, Beech DJ, Wheatcroft SB, Channon KM, Shah AM \& Kearney MT 2013 Nox2 NADPH oxidase has a critical role in insulin resistance-related endothelial cell dysfunction. Diabetes 62 2130-2134. 
Taguchi J \& Freis ED 1975 Partial versus complete control of blood pressure in the prevention of hypertensive complications. Circ Res 36 257-260.

Tchoukalova YD, Krishnapuram R, White UA, Burk D, Fang X, Nijland MJ \& Nathanielsz PW 2013 Fetal baboon sex specific outcomes in adipocyte differentiation at 0.9 gestation in response to moderate maternal nutrient reduction. Int J Obes (Lond) Article ID 23748190, 7 pages, 2013, doi: 10.1038/ijo.2013.106.

Tobi EW, Lumey LH, Talens RP, Kremer D, Putter H, Stein AD, Slagboom PE \& Heijmans BT 2009 DNA methylation differences after exposure to prenatal famine are common and timing- and sex-specific. Hum Mol Genet 18 4046-4053.

Torrens C, Ethirajan P, Bruce KD, Cagampang FR, Siow RC, Hanson MA, Byrne CD, Mann GE \& Clough GF 2012 Interaction between maternal and offspring diet to impair vascular function and oxidative balance in high fat fed male mice. PLoS One 7 e50671.

Touyz RM, Chen X, Tabet F, Yao G, He G, Quinn MT, Pagano PJ \& Schiffrin EL 2002 Expression of a functionally active gp91phox-containing neutrophil-type $\mathrm{NAD}(\mathrm{P}) \mathrm{H}$ oxidase in smooth muscle cells from human resistance arteries: regulation by angiotensin II. Circ Res 90 1205-1213.

Trevenzoli IH, Valle MM, Machado FB, Garcia RM, Passos MC, Lisboa PC \& Moura EG 2007 Neonatal hyperleptinaemia programmes adrenal medullary function in adult rats: effects on cardiovascular parameters. J Physiol 580 629637.

Trudinger BJ, Giles WB, Cook CM, Bombardieri J \& Collins L 1985 Fetal umbilical artery flow velocity waveforms and placental resistance: clinical significance. $\mathrm{Br}$ J Obstet Gynaecol 92 23-30.

Tuomainen AM, Kormi I, Havulinna AS, Tervahartiala T, Salomaa V, Sorsa T \& Pussinen PJ 2012 Serum tissue-degrading proteinases and incident cardiovascular disease events. Eur J Prev Cardiol Article ID 23079500, 8 pages, 2013, doi: 10.1177/2047487312465524.

Turdi S, Ge W, Hu N, Bradley KM, Wang X \& Ren J 2013 Interaction between maternal and postnatal high fat diet leads to a greater risk of myocardial dysfunction in offspring via enhanced lipotoxicity, IRS-1 serine phosphorylation and mitochondrial defects. J Mol Cell Cardiol 55 117-129. 
Valckx SD, De Pauw I, De Neubourg D, Inion I, Berth M, Fransen E, Bols PE \& Leroy JL 2012 BMI-related metabolic composition of the follicular fluid of women undergoing assisted reproductive treatment and the consequences for oocyte and embryo quality. Hum Reprod 27 3531-3539.

van Abeelen AF, Veenendaal MV, Painter RC, de Rooij SR, Dijkgraaf MG, Bossuyt PM, Elias SG, Grobbee DE, Uiterwaal CS \& Roseboom TJ 2012 Survival effects of prenatal famine exposure. Am J Clin Nutr 95 179-183.

van der Steeg JW, Steures P, Eijkemans MJ, Habbema JD, Hompes PG, Burggraaff JM, Oosterhuis GJ, Bossuyt PM, van der Veen F \& Mol BW 2008 Obesity affects spontaneous pregnancy chances in subfertile, ovulatory women. Hum Reprod 23 324-328.

Van Hoeck V, Leroy JL, Arias Alvarez M, Rizos D, Gutierrez-Adan A, Schnorbusch K, Bols PE, Leese HJ \& Sturmey RG 2013 Oocyte developmental failure in response to elevated nonesterified fatty acid concentrations: mechanistic insights. Reproduction 145 33-44.

Van Hoeck V, Sturmey RG, Bermejo-Alvarez P, Rizos D, Gutierrez-Adan A, Leese HJ, Bols PE \& Leroy JL 2011 Elevated non-esterified fatty acid concentrations during bovine oocyte maturation compromise early embryo physiology. PLoS One 6 e23183.

Van Wart HE \& Birkedal-Hansen H 1990 The cysteine switch: a principle of regulation of metalloproteinase activity with potential applicability to the entire matrix metalloproteinase gene family. Proc Natl Acad Sci U S A 87 5578-5582.

Vickers MH, Clayton ZE, Yap C \& Sloboda DM 2011 Maternal fructose intake during pregnancy and lactation alters placental growth and leads to sex-specific changes in fetal and neonatal endocrine function. Endocrinology 152 1378-1387.

Violi F, Pignatelli P, Pignata C, Plebani A, Rossi P, Sanguigni V, Carnevale R, Soresina A, Finocchi A, Cirillo E, Catasca E, Angelico F \& Loffredo L 2013 Reduced atherosclerotic burden in subjects with genetically determined low oxidative stress. Arterioscler Thromb Vasc Biol 33 406-412.

Visse R \& Nagase H 2003 Matrix metalloproteinases and tissue inhibitors of metalloproteinases: structure, function, and biochemistry. Circ Res 92 827-839. 
Vlachopoulos C, Aznaouridis K \& Stefanadis C 2010 Prediction of cardiovascular events and all-cause mortality with arterial stiffness: a systematic review and meta-analysis. J Am Coll Cardiol 55 1318-1327.

Wagner RP 1972 The role of maternal effects in animal breeding. II. Mitochondria and animal inheritance. J Anim Sci 35 1280-1287.

Wakefield SL, Lane M, Schulz SJ, Hebart ML, Thompson JG \& Mitchell M 2008 Maternal supply of omega-3 polyunsaturated fatty acids alter mechanisms involved in oocyte and early embryo development in the mouse. Am J Physiol Endocrinol Metab 294 E425-434.

Wang X, Chow FL, Oka T, Hao L, Lopez-Campistrous A, Kelly S, Cooper S, Odenbach J, Finegan BA, Schulz R, Kassiri Z, Lopaschuk GD \& FernandezPatron C 2009 Matrix metalloproteinase-7 and ADAM-12 (a disintegrin and metalloproteinase-12) define a signaling axis in agonist-induced hypertension and cardiac hypertrophy. Circulation 119 2480-2489.

Wang Y \& Zhao S. 2010. Vascular Biology of the Placenta. San Rafael, CA: Morgan \& Claypool Life Sciences.

Watkins AJ, Lucas ES, Wilkins A, Cagampang FR \& Fleming TP 2011 Maternal periconceptional and gestational low protein diet affects mouse offspring growth, cardiovascular and adipose phenotype at 1 year of age. PLoS One 6 e28745.

Watkins AJ, Platt D, Papenbrock T, Wilkins A, Eckert JJ, Kwong WY, Osmond C, Hanson M \& Fleming TP 2007 Mouse embryo culture induces changes in postnatal phenotype including raised systolic blood pressure. Proc Natl Acad Sci U S A 104 5449-5454.

Watkins AJ, Wilkins A, Cunningham C, Perry VH, Seet MJ, Osmond C, Eckert JJ, Torrens C, Cagampang FR, Cleal J, Gray WP, Hanson MA \& Fleming TP 2008 Low protein diet fed exclusively during mouse oocyte maturation leads to behavioural and cardiovascular abnormalities in offspring. J Physiol 586 22312244.

Watson AL, Skepper JN, Jauniaux E \& Burton GJ 1998 Susceptibility of human placental syncytiotrophoblastic mitochondria to oxygen-mediated damage in relation to gestational age. J Clin Endocrinol Metab 83 1697-1705. 
Wen SW, Leader A, White RR, Leveille MC, Wilkie V, Zhou J \& Walker MC 2010 A comprehensive assessment of outcomes in pregnancies conceived by in vitro fertilization/intracytoplasmic sperm injection. Eur J Obstet Gynecol Reprod Biol 150 160-165.

West NA, Crume TL, Maligie MA \& Dabelea D 2011 Cardiovascular risk factors in children exposed to maternal diabetes in utero. Diabetologia 54 504-507.

WHF. 2012. Cardiovascular disease in children and youth. Retrieved July 20, 2013 from http://www.world-heart-federation.org/fileadmin/user_upload/ documents/Fact_sheets/2012/CVD_children_and_youth.pdf.

Whitten W 1956 Culture of tubal mouse ova. Nature 17796.

WHO 19991999 World Health Organization-International Society of Hypertension Guidelines for the Management of Hypertension. Guidelines Subcommittee. $J$ Hypertens 17 151-183.

WHO. 2006. BMI classification. Retrieved July 24, 2013 from http:// apps.who.int/bmi/index.jsp?introPage=intro_3.html.

WHO. 2013a. The Atlas of Heart Disease and Stroke. Retrieved July 24, 2013 from http://www.who.int/cardiovascular_diseases/resources/atlas/en.

WHO. 2013b. Cardiovascular diseases (CVDs). Retrieved July 23, 2013 from http://www.who.int/mediacentre/factsheets/fs317/en/.

WHO. 2013c. Childhood Overweight and Obesity. Retrieved July 10, 2013 from http://www.who.int/dietphysicalactivity/childhood/en/.

WHO. 2013d. A Global Brief on Hypertension. Retrieved July 23, 2013 from http://apps.who.int/iris/bitstream/10665/79059/1/WHO_DCO_WHD_2013.2_eng. pdf.

WHO. 2013e. Obesity and Overweight. Retrieved July 23, 2013 from http://www.who.int/mediacentre/factsheets/fs311/en/. 
Wikstrand MH, Niklasson A, Stromland K \& Hellstrom A 2008 Abnormal vessel morphology in boys born after intracytoplasmic sperm injection. Acta Paediatr $\mathbf{9 7}$ 1512-1517.

Wildman RP, Mackey RH, Bostom A, Thompson T \& Sutton-Tyrrell K 2003 Measures of obesity are associated with vascular stiffness in young and older adults. Hypertension 42 468-473.

Wilhelm SM, Collier IE, Marmer BL, Eisen AZ, Grant GA \& Goldberg GI 1989 SV40-transformed human lung fibroblasts secrete a 92-kDa type IV collagenase which is identical to that secreted by normal human macrophages. $J$ Biol Chem 264 17213-17221.

Will H, Atkinson SJ, Butler GS, Smith B \& Murphy G 1996 The soluble catalytic domain of membrane type 1 matrix metalloproteinase cleaves the propeptide of progelatinase A and initiates autoproteolytic activation. Regulation by TIMP-2 and TIMP-3. J Biol Chem 271 17119-17123.

Willenbrock F \& Murphy G 1994 Structure-function relationships in the tissue inhibitors of metalloproteinases. Am J Respir Crit Care Med 150 S165-170.

Williams H, Johnson JL, Jackson CL, White SJ \& George SJ 2010 MMP-7 mediates cleavage of N-cadherin and promotes smooth muscle cell apoptosis. Cardiovasc Res 87 137-146.

Wittemer C, Ohl J, Bailly M, Bettahar-Lebugle K \& Nisand I 2000 Does body mass index of infertile women have an impact on IVF procedure and outcome? J Assist Reprod Genet 17 547-552.

Wonnacott KE, Kwong WY, Hughes J, Salter AM, Lea RG, Garnsworthy PC \& Sinclair KD 2010 Dietary omega-3 and -6 polyunsaturated fatty acids affect the composition and development of sheep granulosa cells, oocytes and embryos. Reproduction 139 57-69.

Wrotniak BH, Shults J, Butts S \& Stettler N 2008 Gestational weight gain and risk of overweight in the offspring at age $7 \mathrm{y}$ in a multicenter, multiethnic cohort study. Am J Clin Nutr 87 1818-1824. 
Wu LL, Dunning KR, Yang X, Russell DL, Lane M, Norman RJ \& Robker RL 2010 High-fat diet causes lipotoxicity responses in cumulus-oocyte complexes and decreased fertilization rates. Endocrinology 151 5438-5445.

Xue B, Johnson AK \& Hay M 2013 Sex differences in angiotensin II- and aldosteroneinduced hypertension: the central protective effects of estrogen. Am J Physiol Regul Integr Comp Physiol 305 (5) R459-463.

Yamazaki K, Okada T, Mori Y \& Tanaka I 1998 Genetic mapping of mouse transient receptor potential (Trrp) genes responsible for capacitative calcium entry channels to chromosomes 3, 7, 9, and X. Genomics 51 303-305.

Yang CM, Lee IT, Hsu RC, Chi PL \& Hsiao LD 2013 NADPH oxidase/ROSdependent PYK2 activation is involved in TNF-alpha-induced matrix metalloproteinase-9 expression in rat heart-derived H9c2 cells. Toxicol Appl Pharmacol 272(2) 431-442.

Yang Y, Estrada EY, Thompson JF, Liu W \& Rosenberg GA 2007 Matrix metalloproteinase-mediated disruption of tight junction proteins in cerebral vessels is reversed by synthetic matrix metalloproteinase inhibitor in focal ischemia in rat. J Cereb Blood Flow Metab 27 697-709.

Young LS, KD; Wilmut, I 1998 Large offspring syndrome in cattle and sheep. Human Reproduction 3 155-163.

Yu C, Wang L, Li J, Guo C, Guo X, Zhang X, Xu Y \& Yao Y 2012 Ovarian stimulation reduces IL-6 release from mouse and human pre-implantation embryos. Am J Reprod Immunol 68 199-204.

Zacchigna S, Zentilin L, Morini M, Dell'Eva R, Noonan DM, Albini A \& Giacca M 2004 AAV-mediated gene transfer of tissue inhibitor of metalloproteinases-1 inhibits vascular tumor growth and angiogenesis in vivo. Cancer Gene Ther $\mathbf{1 1}$ 73-80.

Zander-Fox DL, Henshaw R, Hamilton H \& Lane M 2012 Does obesity really matter? The impact of BMI on embryo quality and pregnancy outcomes after IVF in women aged $\leq 38$ years. Australian and New Zealand Journal of Obstetrics and Gynaecology 52 270-276. 
Zegers-Hochschild F, Adamson GD, de Mouzon J, Ishihara O, Mansour R, Nygren K, Sullivan E \& Vanderpoel S 2009 International Committee for Monitoring Assisted Reproductive Technology (ICMART) and the World Health Organization (WHO) revised glossary of ART terminology, 2009*. Fertil Steril 92 1520-1524.

Zhang D, Zhu Y, Gao H, Zhou B, Zhang R, Wang T, Ding G, Qu F, Huang H \& Lu X 2010 Overweight and obesity negatively affect the outcomes of ovarian stimulation and in vitro fertilisation: a cohort study of 2628 Chinese women. Gynecol Endocrinol 26 325-332.

Zhang H, Wang ZW, Wu HB, Li Z, Li LC, Hu XP, Ren ZL, Li BJ \& Hu ZP 2013 Transforming growth factor-beta1 induces matrix metalloproteinase-9 expression in rat vascular smooth muscle cells via ROS-dependent ERK-NF-kappaB pathways. Mol Cell Biochem 375 11-21.

Zollner U \& Dietl J 2013 Perinatal risks after IVF and ICSI. J Perinat Med 41 17-22. 


\section{APPENDIX 1}

\section{Design of Bisulfite Assays for Section of Promoter Region in Mmp2, Mmp7, Mmp9, Mmp14 and Timp1 in Mouse}

While our experimental offspring were being produced, we wanted to design primers to analyze the methylation patterns in the promoter regions of genes implicated in vascular remodeling [i.e. Mmp2, Mmp7, and Mmp9 (Wang et al. 2009, MartinezLemus et al. 2011)], an inhibitor of the MMPs [Timp1 (Itoh \& Nagase 1995)] and another MMP that has been associated with increased body fat [Mmp14 (Chun et al. 2010)]. Proper DNA methylation in the promoter region of genes is important for regulating gene expression (Nagae et al. 2011); therefore, misregulated methylation in the promoter region of a gene could change its transcriptional activity. DNA methylation in mammals primarily occurs in a CpG context (Razin \& Riggs 1980) . I will refer to these CpG occurrences as CpG sites. Due to limited time, DNA methylation analysis has not been performed in our experimental tissues (from Ch. 3), but it will be done in the future.

The tissues used for the design of the bisulfite sequencing primers were frozen aortas from CF1 x B6D2F1/J offspring previously produced in our laboratory. DNA isolation and bisulfite mutagenesis of the DNA was performed as described in (Chen et al. 2013). Gene promoters, except for Mmp9, were identified using the promoter database website (http://rulai.cshl.edu/cgi-bin/TRED/tred.cgi?process=home) and were then aligned to NCBI sequences for each appropriate gene. The promoter for Mmp9 was identified directly through NCBI. A region designed to overlap both the 5' and 3' end of the promoter region was chosen as a region of interest, and this region was used to design 
primer pairs for bisulfite-converted DNA using the Methyl Primer Express Software v1.0, except for Mmp9 and Mmp14 for which I used Bisearch Primer Design and Search Tool (http://bisearch.enzim.hu/?m=search). Several primer pairs were attempted, but the ones that produced the cleanest bands on 1\% agarose gels were optimized as shown below and sequenced to verify that the right product had been amplified. *Note: the agarose gel pictures except for Mmp14 and Timp1 (Fig. A1.1) are shown in C7 tail bisulfite converted DNA, but all primers were also tested in CF1 x B6D2F1/J progeny aorta bisulfite DNA.

Table A1.1 PCR conditions for amplification of promoter regions in mouse bisulfiteconverted DNA

\begin{tabular}{|c|c|c|c|c|c|c|c|}
\hline Gene Symbol & $\begin{array}{c}\text { PCR Annealing } \\
\mathbf{T m}\left({ }^{\circ} \mathbf{C}\right)\end{array}$ & $\begin{array}{c}\text { PCR size } \\
(\mathbf{b p})\end{array}$ & $\begin{array}{c}\text { Primer [] } \\
\mathbf{\mu M}\end{array}$ & $\begin{array}{c}\mathbf{M g C l}_{\mathbf{2}} \text { [] } \\
\mathbf{m M}\end{array}$ & \# Cycles & 2nd PCR & Taq Polymerase \\
\hline Mmp2 & 60 & 569 & 0.3 & 4 & 45 & yes & Go Taq Promega \\
\hline Mmp7 & 59.2 & 512 & 0.3 & 4 & 45 & yes & Go Taq Promega \\
\hline Mmp9 & 61 & 526 & 0.3 & 4 & $\begin{array}{c}45(1 \mathrm{st}) \\
40(2 \mathrm{nd})\end{array}$ & yes & Go Taq Promega \\
\hline Mmpl 4 & 57.3 & 281 & 0.3 & 4 & $\begin{array}{c}45(1 \mathrm{st}) \\
40(2 \mathrm{nd})\end{array}$ & yes & Go Taq Promega \\
\hline Timpl & 59.7 & 362 & 0.3 & 4 & 45 & no & Go Taq Promega \\
\hline
\end{tabular}

[]$=$ concentration, $\mathrm{Tm}=$ temperature $\left({ }^{\circ} \mathrm{C}\right), 2^{\text {nd }} \mathrm{PCR}=2^{\text {nd }} \mathrm{PCR}$ with the template from the first $\mathrm{PCR}$ and the same primers 


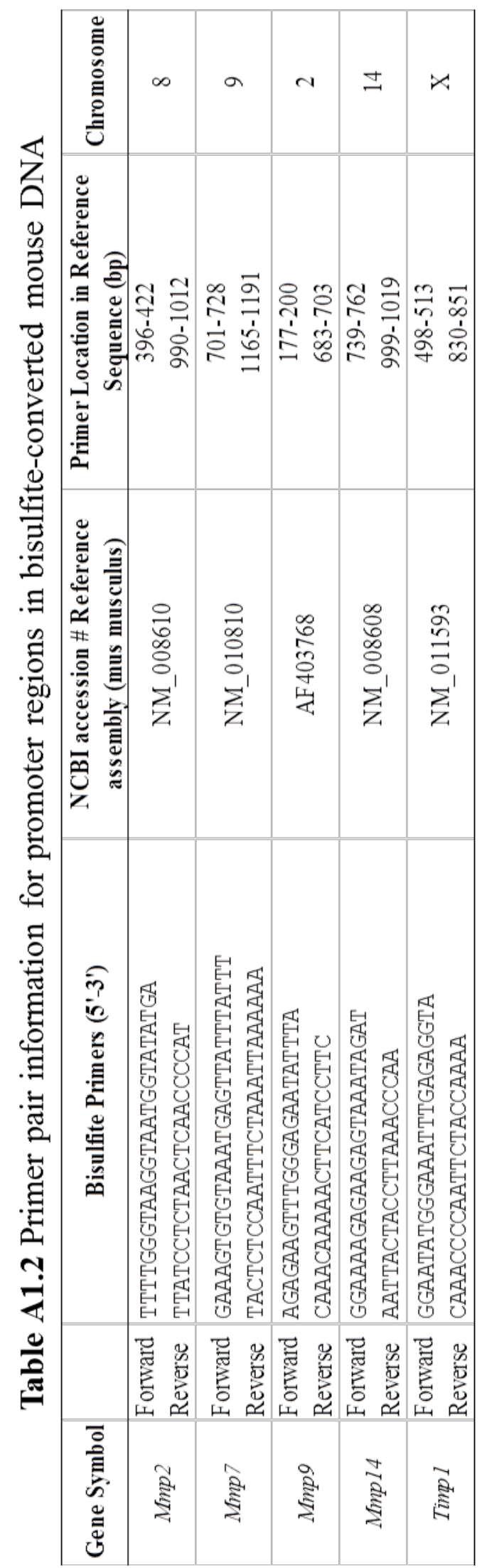



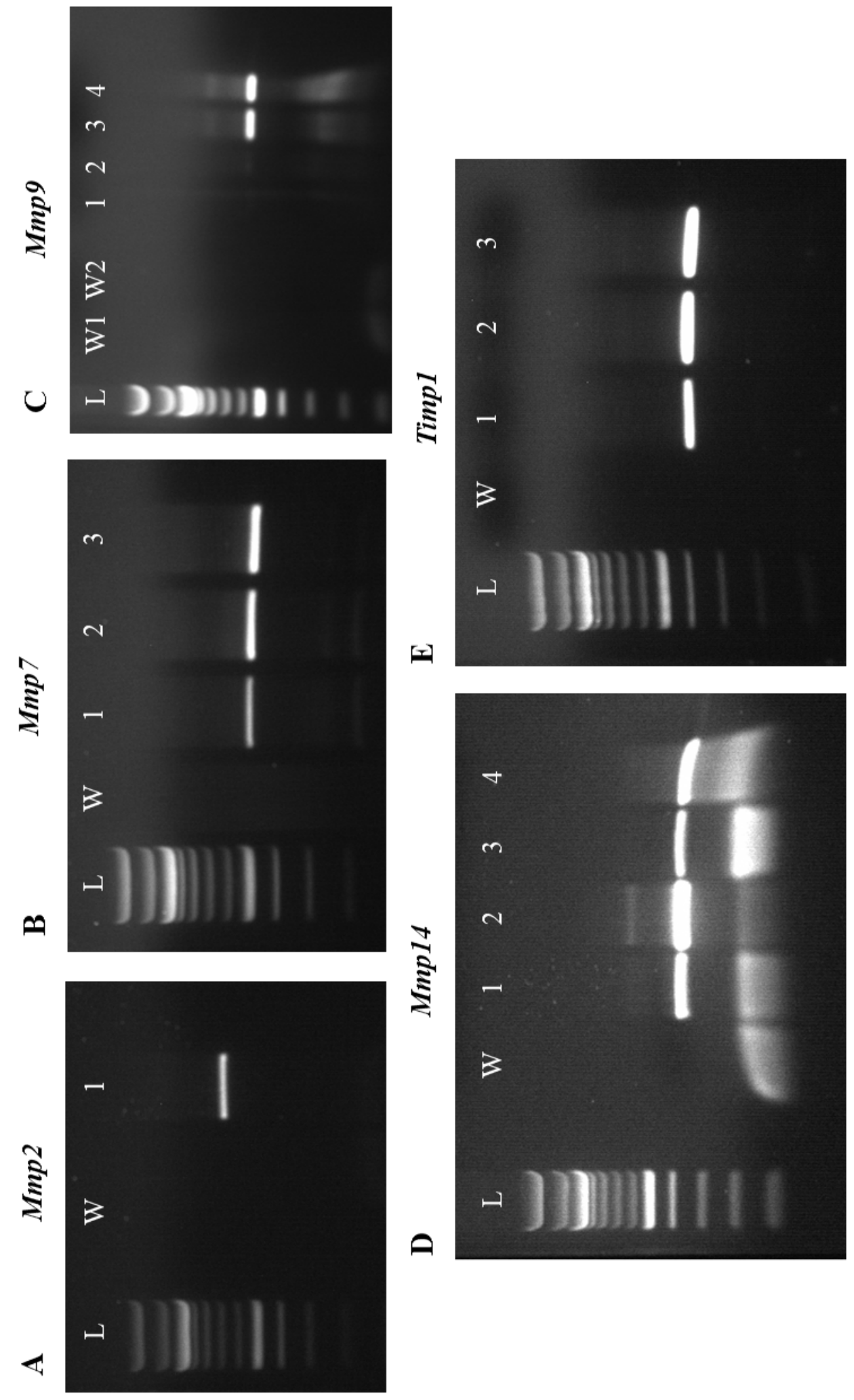

تُ 
Figure A1.1 Amplification of mouse bisulfite converted DNA. Bands represent the amplicon of portions of various promoter regions (i.e. Mmp2, Mmp7, Mmp9, Mmp14 and Timp1) resolved on a 1\% agarose gel. DNA was isolated from tail except when noted. A a 569 bp amplicon containing $10 \mathrm{CpG}$ sites for the $\mathrm{Mmp} 2$ promoter region is shown. W = negative control (water). 1 = C7 tail bisulfite-converted DNA. B shows a 512 bp amplicon containing $8 \mathrm{CpG}$ sites for the $\mathrm{Mmp} 7$ promoter region. $1=\mathrm{C} 7$ tail bisulfiteconverted DNA with $0.2 \mathrm{mM}$ dNTPs. 2 = C7 tail bisulfite-converted DNA with $0.4 \mathrm{mM}$ dNTPs. 3 = C7 tail bisulfite-converted DNA with $0.6 \mathrm{mM}$ dNTPs. C is a 526 bp amplicon containing $10 \mathrm{CpG}$ sites for the Mmp9 promoter . W1 = negative control (water) from $1^{\text {st }}$ PCR. W2 = negative control (water) from $2^{\text {nd }}$ PCR. $1=\mathrm{C} 7$ tail bisulfiteconverted DNA with $0.09 \mu \mathrm{M}$ primers from $1^{\text {st }} \mathrm{PCR} .2=\mathrm{C} 7$ tail bisulfite-converted DNA with $0.09 \mu \mathrm{M}$ primers from $2^{\text {nd }}$ PCR. $3=\mathrm{C} 7$ tail bisulfite-converted DNA with 0.3 $\mu \mathrm{M}$ primers from $1^{\text {st }} \mathrm{PCR} .4=\mathrm{C} 7$ tail bisulfite-converted DNA with $0.3 \mu \mathrm{M}$ primers from $2^{\text {nd }}$ PCR. We varied the amount of primers and tried a single PCR or a nested PCR with the original PCR product as template to see which condition would help us get enough product for sequencing. D shows a $281 \mathrm{bp}$ amplicon containing $27 \mathrm{CpG}$ sites for the Mmp14 promoter region. Lanes 2, 3, 4 and 5 = CF1 x B6D2F1/J aorta bisulfiteconverted DNA. A 362 bp amplicon containing 14 CpG sites for the Timp1 promoter region. E. 1= C7 tail bisulfite-converted DNA. 2 and 3= CF1 x B6D2F1/J aorta bisulfite-converted DNA. L = NEB Ladder N3231S. W = water control (i.e. blank). dNTPs= deoxynucleotide triphosphates. 


\section{VITA}

Angela Louise Schenewerk was born on June 1, 1989 in Kirksville, Missouri. She obtained a Bachelor of Arts in Biology at Westminster College in Fulton, Missouri in May of 2011. During her undergraduate studies she developed her interest in comparative human and animal health through her experiences working in a human cadaver lab, traveling to Belize for a summer to learn about the wildlife, and working at a summer camp with over 100 species of animals. In August of 2011, Angela began her Master of Science studies in developmental epigenetics under Dr. Rocío Rivera in the Division of Animal Sciences and Dr. Luis A. Martinez-Lemus in the Department of Medical Pharmacology and Physiology and the Dalton Cardiovascular Research Center at

the University of Missouri. Upon completion of her M.S. degree, Angela hopes to pursue a career in cancer research or scientific writing. 